\title{
MODEL-BASED STIMULATION OPTIMIZATION FOR CHRONIC PAIN SUPPRESSION USING PERCUTANEOUS AND SURGICAL LEADS IN SPINAL CORD STIMULATION (SCS)
}

Vishwanath Sankarasubramanian 


\section{Members of the Graduation committee:}

Promotor: Prof. Dr. ir. Peter Veltink

Assistant Promotor: Dr. ir. Jan Buitenweg

Members:

Prof. Dr. ir. Kees Slump (University of Twente, Enschede, The Netherlands)

Prof. Dr. ir. Michel van Putten (University of Twente, Enschede, The Netherlands)

Prof. Dr. Bart Nuttin (Katholik University, Leuven, Belgium)

Dr. Ljubomir Manola (Boston Scientific Neuromodulation, Belgium)

The research described in this thesis was performed in the Biomedical Signals and Systems (BSS) group at the University of Twente, Enschede, The Netherlands. In part, this research was financially supported by Boston Scientific Neuromodulation, which is gratefully acknowledged.

Title: Model-based stimulation optimization for chronic pain suppression using percutaneous and surgical leads in spinal cord stimulation (SCS)

Author: Vishwanath Sankarasubramanian

ISBN: 978-94-6191-611-2

Printed by: Ipskamp Drukkers, Enschede, The Netherlands

Copyright $($ 2013, Vishwanath Sankarasubramanian 


\title{
MODEL-BASED STIMULATION OPTIMIZATION FOR CHRONIC PAIN SUPPRESSION USING PERCUTANEOUS AND SURGICAL LEADS IN SPINAL CORD STIMULATION (SCS)
}

\author{
DISSERTATION \\ for the conferral of \\ the degree of Doctor at the University of Twente \\ on the authority of the Rector Magnificus, \\ Prof. dr. ir. Ton J. Mouthaan, \\ in accordance with a decision by the Doctorate Board \\ to be defended in public on
}

Wednesday, January 30, 2013 at 12:45

by

Vishwanath Sankarasubramanian

born on July 1, 1981

in Chennai, India 
This dissertation is approved by:

Promotor: Prof. Dr. ir. Peter Veltink

Assistant Promotor: Dr. ir. Jan Buitenweg 
Dedicated to my parents 



\section{Acknowledgements}

First and foremost, I must acknowledge and thank The Almighty, 'The Divine Mother' for blessing, protecting and guiding me at every point of life.

One of the joys of completion is to look over the journey past and remember all the lovely people, places and events that were part of it. This piece is dedicated to all of them. During my stay in the Netherlands, I learnt a lot about science, but also about myself and life. Infinite support, enthusiasm and optimistic way to deal with life are just few of the many things.

The first debt of sincere and heartfelt gratitude must go to my co-promotor Dr Jan Buitenweg for his constant guidance, support and motivation. He offered me sufficient freedom and space to pursue my research independently and has been an inspirational person in many ways. His mentorship was paramount in providing me a well-rounded experience during these years. I express my deepest gratitude to my promotor, Prof. Dr Peter Veltink for his scientific advice, knowledge, and source of wisdom. He has always been caring and a great leader. Dr Jan Holsheimer is an excellent person who could always be approached for any scientific advice or help. Together with his wife Ria, we prepared delicious cuisines and enjoyed interesting discussions. One person who was ready to help and support me at all times was Wies, the secretary of our group at Biomedical Signals and Systems (BSS). She has been a good friend, well-wisher and a parental figure. Thank you Wies, you are such a vibrant personality!

Special thanks to my committee members, Prof. Dr. ir. Kees Slump, Prof. Dr. ir. Michel van Putten, Prof. Dr. Bart Nuttin, and Dr. Ljubomir Manola for reviewing my thesis and providing encouraging words and constructive feedback.

Adeeb, Peter and later Robert Jan and Lamia were my favorite office mates and friends without whom daily work would not have been so exciting. I was lucky to share the office with you guys and a special mention here to Robert Jan and Lamia for having you as my paranimphs. To all my colleagues at BSS, thank you for being part of those 4 years.

Alizka deserves a special mention for the life that I spent in Enschede and the Netherlands. With your pleasant, polite, and warm personality, you always created a wonderful atmosphere when around. The sincere and lovely times we spent together will always remain fresh in my mind. Thanks from all my heart for your honesty and being the way you are!

To my lovely friends - Merly, Dennis and especially Lamia - the weekend outings that we organized, the many delicious dinners and the parties that we prepared, the interesting topics that we discussed, always 
reflect the good times that we shared together. To my dear friends - Ragav, Avinash, Suresh, Anand, Srikanth, Jithin, Shashank, Dinesh, Vignesh, Hemant, Arun, Sovan and Rahim - thank you for all those eventful parties we had and also for the indoor cricket sessions we organized. You guys are awesome!

Leaving the best to the end - my wonderful and loving family - mom, dad, brother and wife. The reason that I am here and have been able to climb up to this stage today is solely due to my parents - their unconditional love and support. I thank you for your faith in me and allowing me to be as independent and ambitious as I wanted. It was under your watchful eye that I gained so much drive and ability to tackle challenges head on. You have always been there for me and did your best to make my life run as smoothly and happily as possible. Love you so much! My brother is a great friend of mine. I thank him for supporting me throughout. Special thanks to the newest addition to my family, my wife Karpagam, who has given me a new dimension and responsibility to life.

\section{Vishi}

December 2012, Mumbai, India 


\section{Table of contents}

Chapter 1 Introduction to spinal cord stimulation (SCS) - Technical aspects and clinical perspectives

Chapter 2 Triple leads programmed to perform as longitudinal guarded cathodes in SCS - a modeling study

Chapter 3 Electrode alignment of transverse tripoles using a percutaneous

triple lead approach in SCS

Chapter 4 Staggered transverse tripoles with quadripolar lateral anodes using percutaneous and surgical leads in SCS

Chapter 5 Performance of transverse tripoles vs longitudinal tripoles with anode intensification: computational modeling study

Chapter 6 General discussion and final remarks

Summary and Samenvatting

Curriculum vitae

List of publications

125 



\section{CHAPTER 1}

Introduction to spinal cord stimulation (SCS) - Technical aspects and clinical perspectives 
Chapter 1 


\subsection{Spinal cord stimulation (SCS)}

Spinal cord stimulation (SCS) is a well-established electrical neurostimulation technique aimed at alleviating several kinds of chronic pain by means of delivering therapeutic doses of electric current/voltage to the dorsal aspects of the spinal cord, resulting in dermatomal paresthesia and consequent pain relief (1-3).

\subsubsection{Background and mechanisms of action}

SCS was clinically first introduced in 1967 as a neurosurgical treatment for otherwise intractable pain (4), two years after the introduction of the classical Gate-Control theory by Melzack and Wall (5). The stimulator leads were placed subdurally adjacent to the dorsal columns (DCs) of the spinal cord in a patient with terminal cancer and neuropathic pain. Considering the potential for mishap, it is remarkable that not only the surgery was technically successful but also marked pain reduction was experienced by the patient.

The Gate-Control theory, which essentially motivated the first clinical introduction of SCS by Shealy et al., suggests that pain is a complex neurologic and perceptual phenomenon. It postulates that pain perception is a function of the balance between the impulses transmitted to the spinal cord through both the large myelinated nerve fibers and the small pain fibers, both of which synapse at the dorsal horn (Figure 1). Signals from the large myelinated A-beta sensory and small A-delta and C-fibers compete for passage through a physiologic gate. An increase in large nerve-fiber activity could, through interneurons potentially close the gate to signals from small pain fibers entering the dorsal horn. Closing the gate halts the transmission of pain signals to the brain from these small pain fibers. Melzack and Wall hypothesized that preferential electrical stimulation of A-beta fibers would close the gate to pain transmission and reduce the number of pain signals transmitted to the brain. By anatomical coincidence, the large A-beta fibers also ascend in the DCs of the spinal cord. This offers the possibility of stimulating the DCs to promote firing of the large nerve fibers with retrograde transmission down to each segment and subsequent collaterals that enter the spinal cord to close the gate and inhibit pain. 


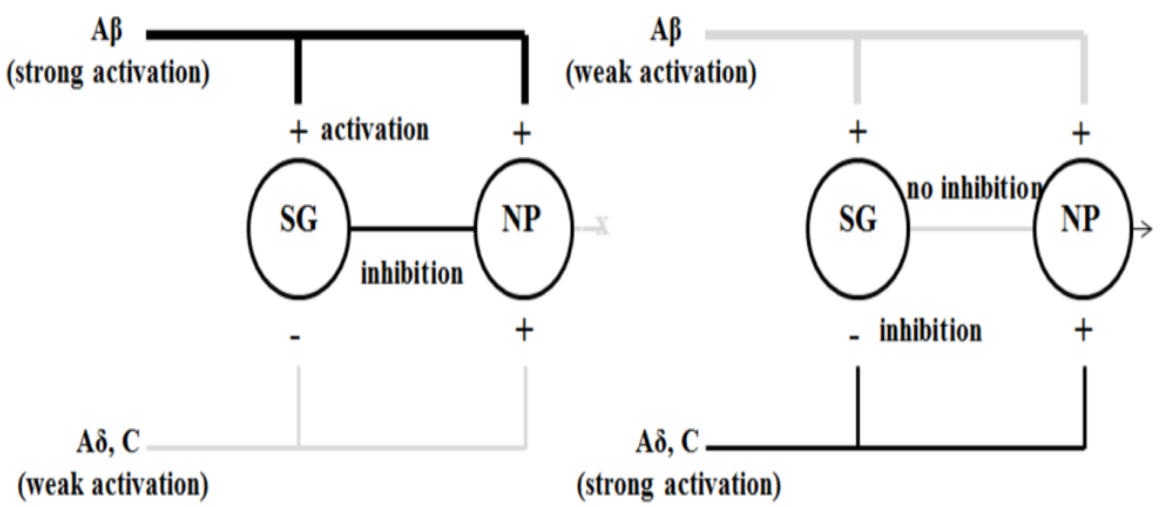

Figure 1 Schematic of the Gate-Control theory as postulated by Melzack and Wall in 1965. NP: Nucleus Proprius; SG: Substantia Gelatinosa. On the left large A-beta fibers (A $\beta)$ are more activated than small fibers, thus activating SG neurons, which in turn inhibit the projection neurons in the NP. In this case, there is no transmission of pain information to the brain. On the right, small nociceptive fibers are more activated than large fibers, inhibiting the inhibitory neurons in the SG and letting the projection neurons send noxious information to the rostral levels of the nervous system. Note: Thick lines denote A fibers and thin lines denote A fibers. Black lines denote high activity and grey lines denote low activity.

Although the Gate-Control theory initially motivated the development of SCS, the exact mechanisms of pain relief or analgesia in SCS are not yet known (6-11). Other theories that have been proposed are

- Stimulation-induced orthodromic propagation of action potentials in the rostral direction to supraspinal centres: A-beta fibers project directly to the DC nuclei and then further connect to the peri-aquaductal grey and the thalamus (12). This in turn might activate descending inhibition resulting in pain relief.

- Stimulation- triggered release of serotonin, substance P and gamma-aminobutyric acid (GABA) within the dorsal horn $(13,14)$. These substances are known to be involved in pain modulation in the spinal cord (10).

- Stimulation-induced blocking of the impulses signalling pain (15).

It is also likely that the analgesia produced in SCS is a result of combination of all or several of these mechanisms of action. All mechanisms involve stimulation of the large A-beta fibers, abundantly found in the DCs, and therefore the scope of this thesis is to assess methods to achieve their preferential stimulation.

Electrical stimulation of the large fibers in the DCs elicits a tingling sensation, called paresthesia (presumably due to orthodromic transmission of the activated DC fibers) that masks the feeling of pain in 
the regions where the pain is felt. An effective SCS therapy should be able to cover the whole extent of the painful regions with paresthesia. Achieving paresthesias in the painful dermatomes is a necessary, although not a sufficient, condition for pain relief (16). It is considered to be a statistically significant predictor of the success of the therapy $(11,17,18)$. The somatotopic organization of the DCs suggests why these fibers are the preferred SCS targets. DC stimulation generates an extensive area of paresthesia coverage, because all DC fibers coming from below the level of the electrode can potentially be activated (19). The distribution of the dermatomes at a low-thoracic level (T11) is depicted below (Figure 2).

Dorsal root (DR) fibers could also yield paresthesia and pain reduction, but presumably only in the corresponding dermatome. Furthermore, the DRs also contain proprioceptive and nociceptive afferents. Activation of these fibers can cause motor activity and therefore discomfort for the patient and should be avoided (2).

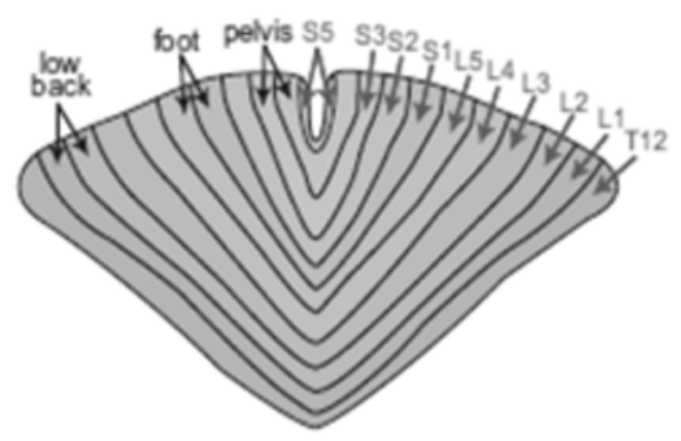

Figure 2 Topographical representation of dermatomes in the dorsal columns of the T11 segment (20).

\subsubsection{Indications for SCS}

The primary indication of SCS is chronic pain, in particular neuropathic pain. Chronic pain is estimated to be the third's largest healthcare problem in the world, afflicting around 30\% of the worldwide population (21). It is a highly debilitating condition, and in particular, is estimated to affect about one-fifth of the population in Europe (18\% in the Netherlands). The impact of chronic pain in the daily life of patients is often significant. It can lead to depression, social isolation and, in the most serious cases, willingness to die (22).

Chronic pain can be classified, according to its mechanism, into (a) nociceptive or (b) neuropathic pain. (a) Nociceptive pain occurs when there is damage near cutaneous afferent fibers, leading to the activation of pain receptors. It normally lasts the period of damage. However, a prolonged activation of these receptors may cause changes in the normal pain pathways (23). (b) In contrast, neuropathic pain appears to emanate from an anatomic region not subject to noxious stimulation, even if the physiologic changes 
sustaining it are not located in that area (24). In physiologic conditions, pain is felt only when a noxious stimulus is carried from peripheral receptors to the brain. However, in neuropathic pain, pain can be felt even in the absence of such stimuli (25). A typically non-nociceptive stimulus (such as touch) can elicit pain and a nociceptive stimulus may induce hyperalgesia, an exaggerated pain sensation. The quality of life of these patients is severely diminished (26).

The management of chronic pain often presents a daunting challenge in clinical practice. The pathophysiology of pain after initial onset becomes much more complex almost immediately - the longer the duration of pain, the more complex the process. Surgical and minimally-invasive techniques for the management of chronic pain have been available for decades. However, neuromodulatory techniques, unlike approaches aimed at selective destruction of the central or peripheral nervous system, are reversible and less likely to be complicated by deafferentation pain. Neuromodulation for chronic pain can be delivered either by means of chemical agents or electrical stimulation.

SCS, which uses electrical stimulation, is a valuable treatment for chronic intractable neuropathic pain. It aims at improving the quality of life of chronic pain patients, by decreasing the pain intensity and substituting it with a tingling paresthesia sensation. In most neuropathic pain states, a paresthesia or tingling sensation must be felt in the affected area for SCS to be effective. However, in patients with deafferentiation or CNS damage, such as brachial plexus avulsion or complete spinal cord injury, it is impossible to produce paresthesia because the necessary neuronal structures have been damaged. Therefore, SCS will hardly be effective in these situations. The Food and Drug Administration (FDA) has approved SCS as a tool in managing chronic, intractable pain of the trunk or limbs, including unilateral or bilateral pain associated with failed back surgery syndrome (FBSS), intractable low-back pain, and leg pain (27-30). Within these indications, the success of SCS varies depending on the type of pain. A higher probability of success has been associated with the following indications: FBSS or post-laminectomy pain, radiculopathy, plexopathy, arachnoiditis, epidural fibrosis, painful peripheral neuropathy, multiple sclerosis and complex regional pain syndrome (CRPS) type 1 (31-36). A reduced probability of success has been associated with the following: axial spine pain associated with FBSS, postherpetic neuralgia, post-thoracotomy pain, phantom pain, intercostals neuralgia and incomplete spinal cord injury $(32,33,35)$.

As we are realizing that many chronic pain conditions constitute an evolving and dynamic process, the new generation SCS equipment is designed to allow the flexibility and complexity that is necessary to maintain long-term pain relief. 


\subsubsection{SCS equipment and current status}

Implanted SCS equipment has advanced considerably over the decades, but has greatly accelerated in their complexity of design in the most recent years. Therefore, the development of SCS equipment must carefully balance the desire to utilize the latest and most advanced electronic components while at the same time provide technology that enables ease of use.

It is estimated that, currently, more than 30,000 SCS equipments are implanted every year worldwide. The three giants of SCS equipments are Medtronic Inc. based in Minnesota, Boston Scientific Corporation, based in California, and St Jude Medical, based in Texas. The equipment consists of three primary components: Implantable pulse generator (IPG), one or more leads housing single or multiple electrodes, and connectors/cables. The IPG houses the stimulation circuitry. The portion of the equipment that is external to the IPG is a solid, interconnected structure consisting of the connectors and the lead(s) housing the electrodes. The basic function of these components is to provide an electrical pathway from the stimulator circuitry to the neural tissue being stimulated. All of these components are designed based on restraints and requirements from both the engineering and clinical realms. Functionally, these components must provide an isolated current pathway, enable adequate tissue activation and selectivity, adequately conform to the anatomy and maintain biocompatibility and reliability throughout the device lifetime.

Lead types: Today's technology allows the implanting physician to deliver effective stimulation to the spinal cord via two types of leads. (1) Percutaneous leads, introduced in the 1970s, are flexible cylindrical polyurethane catheters with multiple, evenly-spaced, cylindrical electrode contacts arranged at the distal end. Some examples of percutaneous leads are Pisces-Sigma, Pisces-Quadripolar and Pisces-Octopolar from Medtronic Inc, Phase 3 Linear from Boston Scientific Corporation, and Quatrode, Octrode from St Jude Medical (Figure 3). The main differences between the mentioned percutaneous leads can be categorized according to the contact length, diameter/width, number of contacts, and contact spacing $(36,37)$. The leads mostly have 4 or 8 contacts and are called quadripolar and octopolar leads respectively (38). Contact spacing varies according to the therapeutic goal (e.g quadripolar electrodes for limb pain and octopolar electrodes for axial pain). Recently, leads with 16 contacts have become available. The Infinion16 lead from Boston Scientific Corporation is a 16-contact percutaneous lead. Percutaneous leads are easy to be implanted and are minimally invasive. Single, dual or triple percutaneous leads can be implanted based on the patient's pain complaint and physician preference. The electrode contacts are composed of platinum alloy (often platinum-iridium) and can be configured as either cathodes or anodes, depending on whether a negative or a positive current/voltage, respectively, is being applied to them. 
Multiple contacts along the lead allow for stimulation field shaping as well as post-implant reprogramming if lead migration occurs (2). The cylindrical design of percutaneous electrode contacts result in circumferential flow of current. Having many contacts increases the need for a strategy to decide for the activation pattern of each of them (there are many combinations and patterns possible). This should be based on a sound understanding of the pathology, the neural activation process, the anatomy of the neural target and the conductivity properties of the surrounding tissue.

Circumferential stimulation has been implicated in painful sensations due to the likely activation of posterior structures in the epidural space of the spinal cord, such as ligamentum flavum. This argument, among others, has been used in support of the preferred use of surgical leads $(9,39,40)$. (2) Surgical leads are flat and wide at the distal end, with up to 16 electrodes placed on one side of a flexible rectangular silicone backing. Some examples of surgical leads are Resume, Symmix, Specify, and Specify 5-6-5 from Medtronic Inc, Artisan from Boston Scientific Corporation, and Lamitrode from St Jude Medical. The main differences between the mentioned surgical leads can be categorized according to the contact length, number of contact columns (one, two, or three), and contact spacing (Figure 3).
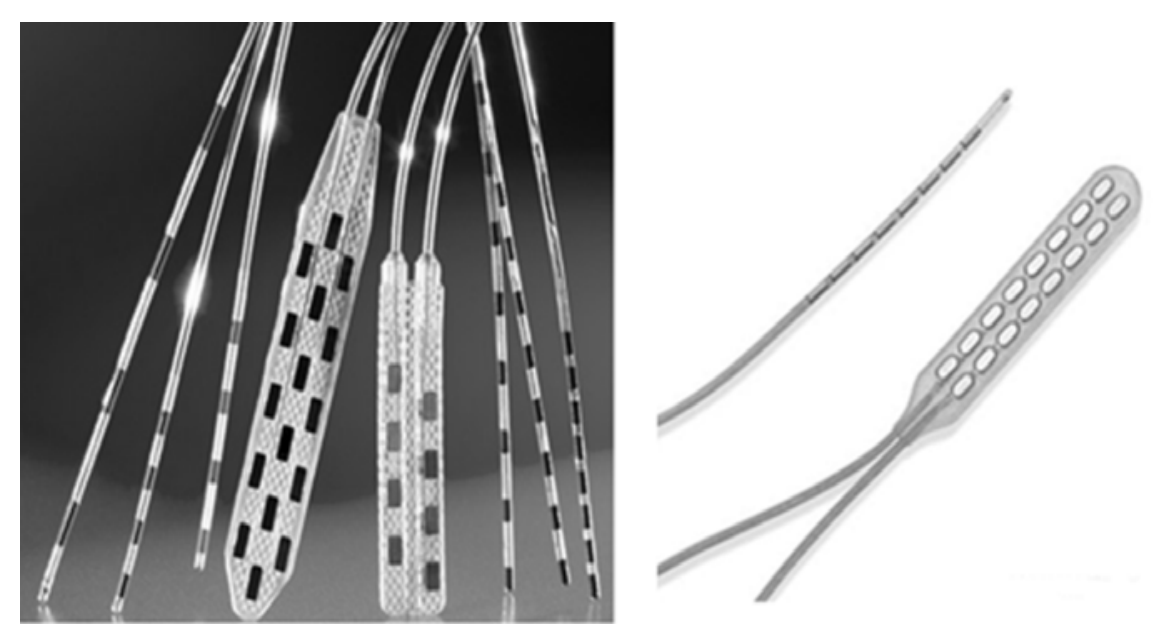

Figure 3 Left: Medtronic percutaneous and surgical leads. Right: Percutaneous and surgical SCS leads by Boston Scientific Neuromodulation (Reproduced from Medtronic, Inc. and Boston Scientific).

The design allows for unidirectional current flow towards the cord, and there is clinical evidence that surgical leads may eliminate discomfort due to the dorsal/posterior structure stimulation sometimes seen with percutaneous leads (39). There are also other advantages of surgical leads compared to percutaneous leads; higher success rates (up to 80-90\%), less long-term migration rates, and better long-term survival $(40,41)$. It has been suggested that increased effectiveness of stimulation and therefore higher success rates of surgical leads can be explained by their relatively large size as compared to percutaneous leads. 
The leads result in compression of the cerebrospinal fluid (CSF) space and thereby bring the electrodes closer to the DCs of the spinal cord (42). Owing to their shape, surgical leads cannot be inserted via a needle and must be surgically implanted.

IPGs: IPG is a component of the SCS equipment responsible for delivering stimulation pulses to the electrode contacts, to which they are connected by connectors/cables (43). Two types of IPGs are currently available: radio frequency (RF) generators and totally implantable battery-powered generators. Totally implantable battery-powered generator contains a rechargeable lithium-ion battery (Figure 4). The advent of rechargeable pulse generators has allowed for more liberal power consumption. Currently available rechargeable batteries are specified to last up to 25 years (different manufacturers claim different longevities), although improvements in battery technology should extend these lifetimes. RF pulse generators equipped with a receiver and an antenna in order to communicate with an energy source (external battery-powered transmitter) are falling out of favour (27). IPGs can either be current- or voltage controlled. Voltage-controlled IPGs, in which the contacts are kept at a constant voltage during stimulation, can potentially have simpler circuitry, can be more power-efficient than current-controlled stimulators, and are better understood than current-controlled stimulators by the clinical community. However, the main drawback of these power sources is that the contact impedance may vary over time, requiring readjustments in the applied voltage (44). The primary advantage current-controlled IPGs offer is direct control over current injection. The stimulation produces an injected current that is independent of the impedance. Moreover, since consumption of energy is one of the factors influencing battery life (nonrechargeable batteries), it is essential that the stimulation current be known. Thus, current-controlled generators are preferable (17). Multichannel pulse generators are of particular interest. These generators have several output channels, allowing independent injection of current or applied voltage via the contacts. This, together with a large number of electrode contacts distributed along the lead, increases the number of possible combinations of injected current or applied voltage (43). Another important aspect of multichannel systems is their reconfigurability. Exact lead placement is often difficult to achieve and can be complicated by anatomical complexities, or the fact that the target is diffuse (pain in multiple dermatomes). Leads with multiple electrode contacts and creative geometries aid in the likelihood of achieving functional outcomes and correcting for suboptimal lead placement (38). Activation of multiple electrode contacts by a grading amount of current injected through the contacts of the same polarity is referred to as current steering. It can be used to increase the selectivity of a given configuration of electrodes by activating tissues that could not be activated by driving the electrodes independently (45). 


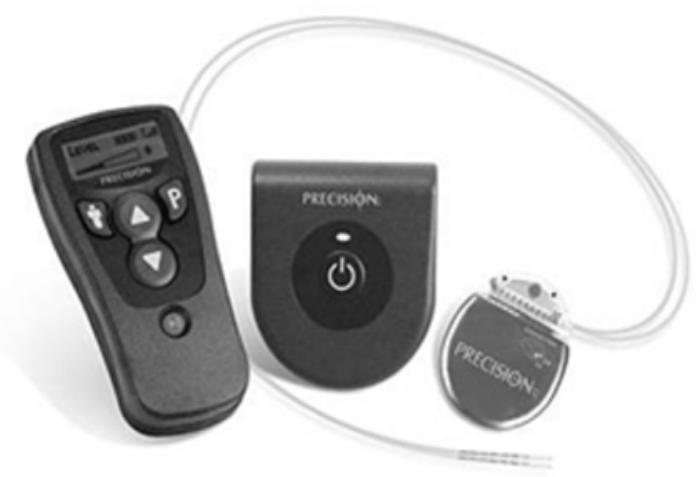

Figure 4 The Boston Scientific Neuromodulation Precision SCS system. On the left is the remote control, in the center is the cordless charger, and on the right is the IPG with two 8-contact epidural leads.

SCS technology has evolved impressively over the past 20 years. Stimulator leads have become more manoeuvrable, which makes them easier to steer within the epidural space (46). The leads now contain more electrodes for greater programming options, including reprogramming in the event of minor lead migration (46). Pulse generator technology has advanced as well. IPGs have become smaller, with much greater programming capabilities (36).

\subsubsection{SCS procedure and efficacy}

The primary purpose of SCS is to reduce the frequency, duration, and intensity of pain $(34,47)$. As in any treatment, the success of SCS depends on appropriate patient selection. Patients should undergo a thorough evaluation, including a detailed history and physical examination, as well as diagnostic and imaging studies. One of the major advantages to SCS is that of conducted trial stimulation that provides information about the potential technical and clinical success of the therapy. The trial depends on the ability to successfully place the percutaneous leads within the epidural space of the spinal cord (Figure 5). During the trial, with the patient under local anaesthesia and prone in a fluoroscopy procedure suite, intraoperative stimulation testing is performed with a combination of electrodes, at least one of which is an anode and the other a cathode. Identifying the effective amplitude range is accomplished by gradually increasing the stimulation until the patient first reports paresthesia. Various combinations of anodes and cathodes, frequency, and pulse width are attempted and varied until a paresthesia covering the entire pain area is achieved. 


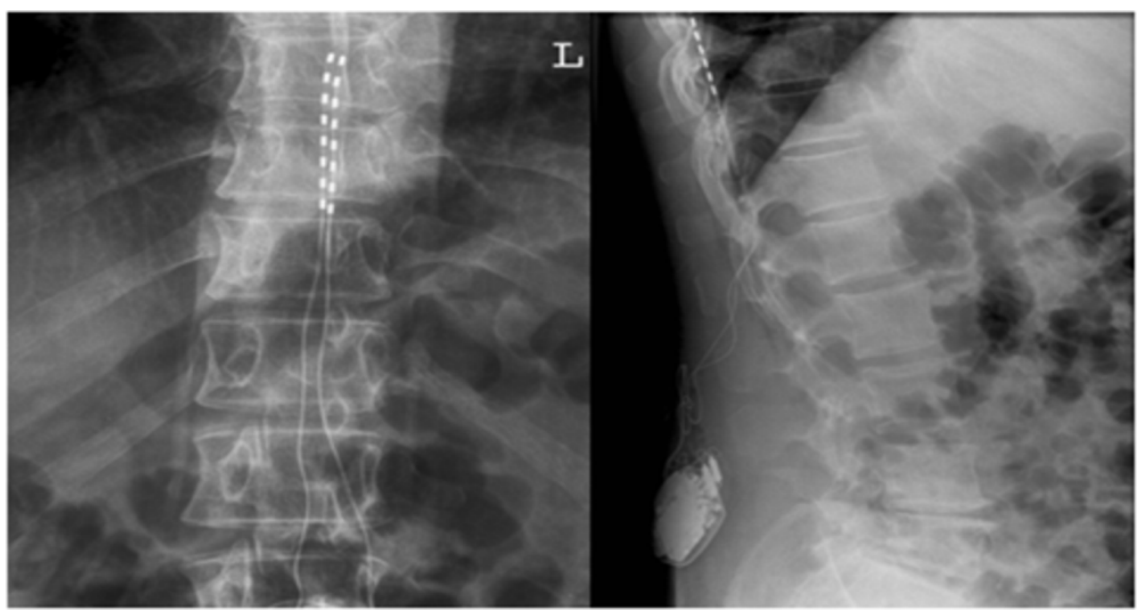

Figure 5 Percutaneously inserted epidural electrodes including IPG

With recent advances in technology employing joystick manipulation of current, programming is often performed rapidly. The programming units of these newer systems contain internal algorithms for electronically trolling down the lead using combinations of anodes and cathodes, making it easy to rapidly cycle through hundreds of combinations in a relatively short time. It is important to note that if the trial is performed under general instead of local anaesthesia, it is difficult for the implanting physician to achieve optimal lead positioning. The physician has to rely on radiographic positioning of the electrodes and/or somatosensory evoked potentials (SSEP). Moreover, it is difficult to assess whether uncomfortable motor effects occur during stimulation. Also, since dermatomal paresthesia coverage is a prerequisite for successful treatment, such a feedback is impossible with general anesthetized patients. If pain is markedly reduced (more than 50\%) during the trial period (usually ranges from 3-8 days), permanent implantation is performed (48). Compared with alternative surgical procedures for pain, SCS is less invasive and less disruptive because it does not ablate pain pathways or result in anatomic change (46). As an augmentative procedure, SCS is reversible and offers patients the opportunity of undergoing the screening trial with a temporary SCS system prior to implantation. This screening trial provides an idea of the implantation and a possible result and, thus, generates an advantage not shared by anatomic or ablative prognostic procedures (e.g., reversible local anaesthetic blockade to predict response to nerve section).

The efficacy of SCS has been well documented in the literature over the past 40 years, especially for neuropathic low-back and leg pain. More than 500 clinical trials, 38 of them randomized controlled trials, have been conducted on SCS, since 1973 (38). Appropriate pain relief, reduced utilization of health care resources, increased activities of daily living (ADL), and reduced medication requirement, potentially leading to improved neurologic and cognitive functioning are some of the common end points used in SCS efficacy studies. By these criteria success rates of $50 \%$ to $70 \%$ are common (49). There is no 
conclusive evidence that SCS is effective in treating nociceptive pain unless it is secondary to ischemia. SCS also appears to be more effective in treating extremity or radicular pain than axial, midline pain even when the axial pain is neuropathic, which is common after back surgery. As promising as SCS has become, $20 \%$ to $40 \%$ of patients still report loss of analgesia within 24 months of implantation (50). Growing anecdotal evidence suggests that when this loss of analgesia occurs, it can often be remedied by re-implantation of a new IPG with improved electrode configurations, intelligent contact combinations and robust programming capabilities. Further clinical studies are necessary to confirm these observations.

\subsection{Challenges faced from a clinical perspective}

Individual patients considering SCS may have exhausted conventional, pharmacologic, complementary, and manipulative therapies. SCS has emerged as a last-resort effective pain therapy in chronic neuropathic pain states. Despite being a widely used technique that has gone through an enormous technological revolution over the last four decades, many challenges regarding the clinical and technical effectiveness of the SCS therapy are yet to be overcome. Some of the important ones are listed and explained below. The question is how to further improve the effectiveness of the therapy, especially as related to the still significant failure rate of $30 \%$ (27). The question is addressed, where the current understanding of some of the technical and clinical aspects of SCS is reviewed, with recommendations for further improvements that may enhance the effectiveness of the therapy.

\subsubsection{Choice of stimulation - Current/voltage controlled and Single/multiple source}

In the electrical excitation of nervous tissue, it is the current through and not voltage at the electrodes that determines the population of neurons excited (51). Lead movement and tissue growth over time would change the impedance seen by the pulse generator and thus the current delivered to the tissue, thus changing the resultant clinical effect of the implanted system. Hence, devices that control current directly are under an advantage. Added to this is the design deficiency of utilizing a single stimulation source and multiplexing it to multiple electrode contacts. When multiple contacts are connected in parallel with a single voltage source, their individual electrode tissue impedances would determine the distribution of current to the nearby neurons. Hence, current cannot be predicted to be divided uniformly among the connected electrodes. Other systems that deliver current source stimulation with an increased number of stimulation contacts still use multiplexed connection of the pulse generator source to the electrode contacts. While it is possible to exactly control the current delivered to a nerve using a single contact, when multiple contacts are connected together using the multiplexer, the distribution of current is actually controlled by the electrode/tissue impedance. Hence, controlled distribution of current through multiple contacts cannot be achieved with single-source. This, again, results in a severe clinical limitation. 
A design utilizing multiple independent current control (MICC) is the only way in which the amount of current delivered to each contact can be precisely controlled. This has the added benefit that non-uniform current distributions can be obtained, uniquely delivering the required stimulation energy to each population of nerves adjacent to the electrode contacts. This means that multiple regions of the spinal cord can be stimulated with their own unique stimulation parameters. This can overcome the effects of variable fibrosis formation, potentially reducing the incidence of discordant paresthesia (17).

\subsubsection{Lead choice and number of lead(s)}

In the 1970s, clinicians developed the percutaneous method of inserting temporary catheter leads for use in the SCS screening trial, with the expectation that permanent surgical lead implantation would occur via laminectomy $(52,53)$. Soon thereafter, adoption of these percutaneous techniques for permanent implantation yielded results approaching those achieved with surgical techniques (54). Indeed, the majority of SCS procedures are currently performed by anaesthesiologists and must rely on use of percutaneous leads.

The decision whether to place one, two or three percutaneous leads depends on both the pain condition that is being treated and physician preference. Placement of a single quadripolar lead at various mediolateral positions in the epidural space is used to treat unilateral and bilateral pain complaints. If the patient has unilateral extremity pain, the lead is placed a few millimetres off the midline, ipsilateral to the painful extremity. If the patient has bilateral extremity pain, which is commonly seen, placement of a single midline lead is attempted in hope that bilateral stimulation would result in balanced paresthesias in both extremities. Unfortunately, lead migration is and continues to be the most common equipment-related complication hindering accurate stimulation paresthesias (55). With the development of systems that can deliver stimulation using two leads, many physicians now routinely prefer dual leads, for the following reasons: (1) in the event of lateral lead migration, stimulation can be electronically transferred horizontally (either medially or laterally) between the leads to recapture the sweet spot, (2) in patients with bilateral extremity pain, placing each lead slightly off the midline greatly facilitates the perception of stimulation evenly felt in both extremities (56-58). Moreover, as has been shown in computer modeling studies, dual leads placed next to each other, straddling the physiologic midline can superimpose the electric fields effectively and achieve ample penetration into the midline of the DCs (59). The earliest experiments with three implanted percutaneous leads were performed by Prager and Chang. They evaluated the effect of balancing the current between a central lead and two lateral leads in a patient with FBSS (60). In 2007, Medtronic researchers released a white paper describing the results of the computer modelling of different triple-lead configurations (61). In 2008, a patient with FBSS was implanted with a 
transverse tripolar system consisting of a cathode surrounded by anodes, in a triple-lead configuration using voltage-controlled electrodes. Pain relief was estimated to be more than $70 \%$ and was maintained for a year (62).

The choice of surgical leads have become a necessity in patients in whom anatomy prevents percutaneous lead placement; when repeated lead revisions are required due to displacement or fracture; or when change in distribution of paresthesia occurs that cannot be recaptured with percutaneous lead revision. Surgical leads are also useful in situations when a percutaneous lead fails to achieve the desired paresthesia coverage during trial stimulation. Surgical leads are also gaining popularity in situations where axial pain is more predominant than radicular pain $(40,58)$.

\subsubsection{Lead positioning and choice of electrode contact combinations}

A prerequisite for effective chronic pain management is to direct the stimulation-generated paresthesias to the painful areas, which is often difficult to achieve because of difficulties in optimal lead positioning. Several empirical and theoretical computer modeling studies were performed in order to obtain a more thorough understanding of factors determining optimal lead positioning $(63,64)$. The problem of optimal lead positioning can potentially be solved by increasing the number of electrode contacts; thereby increasing contact points and contact combinations and thus the probability of generating effective paresthesias. In particular, the choice of contact combinations on lead(s) can have different intended clinical effects. It was shown previously that differential activation of DC and DR fibers strongly depends on the anode-cathode combinations (mono-, bi-, tripolar stimulation) and on their geometry (length and longitudinal distance between contacts) (1). Bipolar stimulation favoured the activation of DC fibers, whereas single cathode stimulation preferentially excited DR fibers. When comparing bi- with tripolar stimulation (in a guarded cathode configuration: anode-cathode-anode), it was predicted that the latter would yield even better results in terms of DC activation (2). The theoretically predicted superiority of a guarded cathode configuration over mono- and bipolar approaches has been confirmed in clinical trials (16).

Longitudinal guarded cathode (+-+) configurations are useful in areas in which the sweet spot is narrow and stimulation outside the sweet spot results in activation of unwanted structures (57). It is believed that, such a focussed stimulation can also be achieved by transverse tripolar configurations using both, surgical and percutaneous leads. New-generation leads using several columns of stimulation electrodes also effectively generate longitudinal and/or transverse stimulation fields into the spinal cord. The leads are believed to improve target selectivity in stimulation necessary for relieving certain difficult-to-treat pain conditions, such as low-back pain. 


\subsubsection{Complexity of low-back stimulation}

In the average patient, it is more difficult to achieve paresthesia overlap of low-back pain than of radicular leg pain (65). The low-back area remains difficult to stimulate without intervening chest or abdominal wall stimulation. A number of other factors which underlie the relative difficulty of stimulating the lowback include cord diameter, CSF thickness, and topographic organization of nerve fibers (66). Stimulating the low-back, usually at dermatomes between L2 and L5, is most often accomplished by placing the lead tips at the midline of T8 to T9. Initially, Law $(63,65)$ showed that the low-back fibers may be more selectively activated by a matrix of closely-spaced electrodes at the T9-T10 spine level (Figure 6).

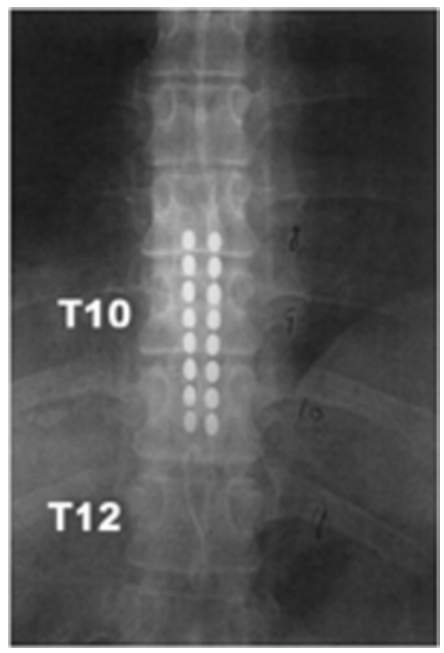

Figure 6 Optimal electrode construct to maximize stimulation of the lower lumbar area. AnteroPosterior x-ray of the thoracic spine.

Various percutaneous lead configurations are currently being used for low-back pain treatment. Some physicians use a single percutaneous quadripolar lead on the physiological midline $(10,63)$. They postulate that patients can tolerate high amplitudes with this configuration because the electrodes are relatively distant from the DR fibers. Others prefer dual percutaneous quadripolar leads flanking the midline, which may create paresthesia in both the back and lower limbs, resulting in better coverage (67). A third configuration uses triple percutaneous lead arrays. Prager et al reported a system consisting of 3 percutaneous leads: 1octopolar lead on the midline in between 2 flanking quadripolar leads connected in parallel (60). However, optimal outcomes are served only by those configurations which are able to effectively direct current, since the area of the DC that produces precise dermatomal coverage when stimulated, known as the sweet spot, can occupy a relatively small area, particularly in low-back stimulation. 
Neurostimulation has currently not been validated in the treatment of back pain because of technological limitations in implantable SCS systems. The lack of validated technique for low-back pain relief has prompted the development of newer design of leads, including leads with increased number of contacts (up to 16) and various geometric arrangements, the objective of which is to cover a large area while attempting to extend, steer, or focus the electric field of the stimulation within the spinal cord regions (68).

\subsection{Computer modeling in SCS}

Computer modeling of neurostimulation is an effective tool to assist in the understanding of the complex interactions between electric fields and the spinal cord. Because many of these interactions are especially difficult to characterize with traditional experimental techniques, computer models play an increasingly important role in the scientific analysis of neurostimulation. Only clinical studies and long-term follow-up can prove safety of a clinical technique/system. Before embarking on a clinical trial, all kind of safety aspects, numerous tests (of which computer modeling is a part) are performed-clinical studies in human patients might not be ethically acceptable if what is being tested has not yet been proven safe. An alternative is to perform experiments in animals. However, not knowing if and how exactly the results can be extrapolated to humans is most likely a source of bias and thus a major drawback. One way of addressing these limitations is to use computer models that mimic the behaviour of spinal cord structures. Hence, it must be understood that computer modelling, is not a sole factor contributing to the safety of an implantable device/clinical trial. It is a valuable tool to predict the effect of electrical stimuli on the activation of neural structures and to help in the design of more effective stimulation parameters.

\subsubsection{The University of Twente Spinal cord stimulation (UT-SCS) model}

With the aim of better understanding the effect of electrical stimulation on nerve fiber activation, several computer models mimicking SCS have been developed in the past few decades $(69,70)$. The University of Twente group introduced a more complex and accurate model, named the UT-SCS model (71). The UTSCS model consists of two interconnected parts: (a) volume conductor model and (b) nerve fiber model (Chapter 2-Chapter 5). (a) The volume conductor model represents both the geometry and the electrical conductivities of the constituting anatomical structures at three different spine levels. Additionally, the stimulation leads are modelled in the dorsal epidural space, in which voltage or current can be applied. The tissue conductivities were either obtained from the literature or from measurements and approximation techniques (Chapter 2). The intra-vertebral geometries were based on earlier human MRI studies (72). After the discretization of the volume conductor model, a finite differences method is applied. Poisson's equation is solved to obtain electrical potentials at all the grid nodes of the model. (b) 
The nerve fiber model uses a McNeal fiber model extended with collaterals, for representing DC fibers (73). Curved fibers were later introduced for modeling DR fibers and therefore an improved fiber model was used (74). Several fiber parameters are defined in the UT-SCS model: the fiber diameter, the number of nodes of Ranvier, the nodal area and the number and the position of collaterals branching from a longitudinal fiber (Chapter 4).

The UT-SCS model is used to simulate the stimulation-induced electric field and the response of myelinated nerve fibers $(73,75)$. The model allows the design of an optimum electrode geometry, contact separation, contact size and configuration for SCS under various stimulation conditions with a longitudinal and/or transverse contact array, both surgical and percutaneous (2). The development of the UT-SCS model has led to the following recommendations and clinical validations for human longitudinal contact array electrodes. (1) The contact center-center separation is the most critical parameter and should be between 4 and $4.5 \mathrm{~mm}$ (2). (2) Minimal electrode contact surface should be $6 \mathrm{~mm}^{2}$, according to FDA regulations regarding maximum current density and maximum charge/pulse (76). (3) The contact length should be between 1.5 and $3 \mathrm{~mm}$ (2). (4) When using a surgical lead, the contacts should be approximately $4 \mathrm{~mm}$ wide.

\subsection{Objectives of the thesis}

The target neurons in the DCs of the spinal cord are aimed to be electrically stimulated in order to provide an optimal relief of pain. The SCS electrode is the interface between the electrical signal of the stimulator and the nerve fibers of the target DCs. As mentioned in the previous section, the UT-SCS model has been used effectively to drive the design of stimulating electrodes/leads. As a potential improvement, this thesis presents the clinical and technical aspects of stimulation optimization techniques for chronic pain relief in SCS. The optimization techniques are aimed to focus primarily on improving SCS equipment. In particular, the thesis investigates the performance of novel percutaneous and surgical triple-lead configuration designs, with both longitudinal and transverse tripolar contact combinations, in a currentcontrolled stimulation approach. Effects of percutaneous lead alignment/misalignment, varied transversal lead spacing, preferred choice of leads (surgical/percutaneous), and IPG design are also modeled as ways to potentially improve SCS equipment.

\subsection{Outline of the thesis}

In this thesis, stimulation optimization using computer modeling of percutaneous and surgical leads for chronic pain relief in SCS is presented. In chapter 2, triple percutaneous leads programmed to function as longitudinal guarded cathodes are modeled as a potential improvement to dual leads commonly used in 
clinical practice. The effect of transversal lead separation and anodal current steering mechanisms using a triple lead guarded cathode configuration on the medio-lateral extent of DC coverage is studied. Also, the post-operative flexibilities of single, dual and triple lead longitudinal guarded cathode configurations are compared. Electrode alignment of transverse tripoles using a percutaneous triple lead approach is modeled in chapter 3 . The influence of electrode alignment of the transverse tripoles on the paresthesia coverage of pain area is presented. Aligned and staggered triple leads are modeled and transverse tripolar stimulation is performed to investigate the effects of the above configurations on the DC recruited area. In chapter 4, transverse tripolar configurations using quadripolar instead of dual anodes are modeled both using percutaneous and surgical leads. The additional anodal contacts are programmed to understand the stimulation effects on DC fiber selectivity and shielding of DR fibers. The effect of contact spacing and insulation is determined by comparing the performance of the percutaneous and surgical triple lead transverse tripolar configurations with quadripolar anodes. Chapter 5 introduces and investigates anode intensification effects on the performance of transverse tripolar and longitudinal tripolar configurations. Anodal currents are increased with respect to the cathode to determine the effects of stimulation on DC recruitment and usage ranges. 


\section{References}

[1] Holsheimer J, Struijk JJ, Tas NR. Effects of electrode geometry and combination on nerve fiber selectivity in spinal cord stimulation. Med Biol Eng Comput. 1995;33:676-682.

[2] Holsheimer J, Wesselink WA. Effect of anode-cathode configuration on paresthesia coverage in spinal cord stimulation. Neurosurgery. 1997;41:654-659.

[3] Struijk JJ, Holsheimer J, Boom HBK. Excitation of dorsal root fibers in spinal cord stimulation: a theoretical study. IEEE Trans Biomed Eng. 1993;40:632-639.

[4] Shealy CN, Mortimer JT, Reswick JB. Electrical inhibition of pain by stimulation of the dorsal columns: preliminary clinical report. Anesth analg. 1967;46:489-491.

[5] Melzack R, Wall PD. Pain mechanisms: a new theory. Science. 1965;150:971-978.

[6] Gybels J, van Roost D. Spinal cord stimulation for the modification of dystonic and hyperkinetic conditions: A critical review. In: Recent Achievements in Restorative Neurology; Book 1: Upper motion neuron functions and dysfunctions. Basel: Karger, 1985:56-70.

[7] Krames ES. Mechanisms of action of spinal cord stimulation. In: Waldman SD. Interventional Pain Management. Philadelphia: WB Saunders, 2001:561-565.

[8] Meyerson B, Linderoth B. Spinal cord stimulation: mechanisms of action in neuropathic and ischaemic pain. In: Simpson BA. Electrical stimulation and the Relief of Pain (Pain research and clinical management series). Elsevier, 2003:161-182.

[9] North RB, Kidd DH, Olin J, Sieracki JM, Farrokhi F, Petrucci L et al. Spinal cord stimulation for axial low back pain: a prospective, controlled trial comparing dual with single percutaneous electrodes. Spine. 2005; 30:1412-1418.

[10] Oakley JC, Prager JP. Spinal cord stimulation: Mechanisms of action. Spine. 2002;27:2574-2583.

[11] Simpson BA. Spinal cord stimulation. Pain Rev. 1994;1:199-230.

[12] Nyquist JK, Greenhoot JH. Responses evoked from the thalamic centrum medianum by painful input: suppression by dorsal funiculus conditioning. Exp Neurol. 1973;39:215-222.

[13] Linderoth B, Grazelius B, Franck J and Brodin E. Dorsal column stimulation induces release of seretonin and substance $\mathrm{P}$ in the cat dorsal horn. Neurosurgery. 1992;31:289-297.

[14] Linderoth B, Stiller CO, Gunasekara L, O’Connor WT, Ungerstedt U, Brodin E. Gamma aminobutyric acid is released in the dorsal horn by electrical spinal cord stimulation: an in vivo microdialysis study in the rat. Neurosurgery. 1994;34:484-489.

[15] Campbell JN. Examination of possible mechanisms by which stimulation of the spinal cord in man relieves pain. Appl Neurophysiol. 1981;44:181-186. 
[16] North RB, Ewend MG, Lawton MT, Piantadosi S. Spinal cord stimulation of chronic, intractable pain: superiority of 'multi-channel' devices. Pain. 1991;44:119-130.

[17] Aló KM, Redko V, Charnov J. Four year follow-up of dual electrode spinal cord stimulation for chronic pain. Neuromodulation. 2002;5:79-88.

[18] Barolat G. current status of epidural spinal cord stimulation. (Review). Neurosurg Q. 1995;5:98-124.

[19] Feirabend HKP, Choufoer H, Ploeger S, Holsheimer J, Van Gool JD. Morphometry of human superficial dorsal and dorso-lateral column fibres: significance to spinal cord stimulation. Brain. 2002;125:1137-1149.

[20] Holsheimer J. Does dual lead stimulation favor stimulation of the axial lower back? (Editorial). Neuromodulation. 2000;3:55-57.

[21] Latham J, Davis BD. The socioeconomic impact of chronic pain. Disability and Rehabilitation. 1994;16:39-44.

[22] Breivik H, Collett B, Ventafridda V, Cohen R, Gallacher D. Survey of chronic pain in Europe: prevalence, impact on daily life and treatment. Eur J Pain. 2006;10:287-333.

[23] Koltzenburg M, Torebjork HE, Wahren LK. Nociceptor modulated central sensitization causes mechanical hyperalgesia in acute chemogenic and chronic neuropathic pain. Brain. 1994;117:579591.

[24] Baron R. Mechanisms of disease: neuropathic pain-a clinical perspective. Nat Clin Pract Neurol. 2006;2:95-106.

[25] Backonja MM, Galer BS. Pain assessment and evaluation of patients who have neuropathic pain. Neurologic Clinics. 1998;16:775-789.

[26] Fazen LE, Ringkamp M. The pathophysiology of neuropathic pain: a review of current research and hypothesis. Neurosurgery. 2007;17:245-262.

[27] Barolat G. Spinal cord stimulation for chronic pain management. Arch Med Res. 2000;31:258-262.

[28] Sundaraj SR, Johnstone C, Noore F, Wynn P, Castro M. Spinal cord stimulation: a seven year audit. J Clin Neurosci. 2005;12:264-270.

[29] Taylor RS. Spinal cord stimulation in complex regional pain syndrome and refractory neuropathic back and leg pain/failed back surgery syndrome: results of a systematic review and meta-analysis. $J$ Pain Symptom Manage. 2006;31(4 Suppl):S13-9.

[30] Kumar K, Malik S, Demeria D. Treatment of chronic pain with spinal cord stimulation versus alternative therapies: cost-effectiveness analysis. Neurosurgery. 2002;51:106-115.

[31] Augustinsson LE. Spinal cord electrical stimulation in severe angina pectoris: surgical technique, intraoperative physiology, complications and side effects. Pacing Clin Electrophysiol. 1989;12:693694. 
[32] Kumar K, Toth C, Nath R, Laing P. Epidural spinal cord stimulation for treatment of chronic pain: some predictors of success. A 15 year experience. Surg Neurol. 1998;50:110-121.

[33] Kumar K, Hunter G, Demeria D. Spinal cord stimulation in treatment of chronic benign pain: challenges in treatment planning and present status, a 22-year experience. Neurosurgery. 2006;58:481-496.

[34] Lee AW, Pilitsis JG. Spinal cord stimulation: indications and outcomes. Neurosurg Focus. 2006;21:E3.

[35] Simpson A, Meyerson BA, Linderoth B. Spinal cord and brain stimulation. In: McMahon SB, Koltzenburg M, eds. Wall and Melzack's textbook of pain, $5^{\text {th }}$ ed. Philadelphia: Elsevier Churchill Livingstone;2006:569.

[36] Barolat G, Sharan AD. Spinal cord stimulation for chronic pain management. Seminars in Neurosurgery. 2004;15:151-175.

[37] Simpson BA, Bassett G, Davies K, Herbert C, Pierri M. Cervical spinal cord stimulation for pain: a report on 41 patients. Neuromodulation. 2003;6:20-26.

[38] Kries PG, Fishman SM. Spinal cord stimulation: percutaneous implantation techniques. Oxford: Oxford University Press. 2009.

[39] North RB, Lanning A, Hessels R and Cutchis PN. Spinal cord stimulation with percutaneous and plate electrodes: side effects and quantitative comparisons. Neurosurg. Focus.1997;2:1-5.

[40] North RB, Kidd D H, Olin JC and Sieracki JM. Spinal cord stimulation electrode design: prospective, randomized, controlled trial comparing percutaneous and laminectomy electrodes-part I: technical outcomes. Neurosurgery. 2002;51:381-90.

[41] Villavicencio A, Leveque J, Rubin L, Bulsara K and Gorecki J. Laminectomy versus percutaneous electrode placement for spinal cord stimulation Neurosurgery. 2000;46:399-406.

[42] Law JD. Percutaneous spinal cord stimulation for the failed back surgery syndrome. Pain manage. 1991;1:1-2.

[43] North RB. Neural interface devices: Spinal cord stimulation technology. IEEE Trans Biomed Eng. 2008;7:1108-1119.

[44] Oakley J, Varga C, Krames E, Bradley K. Real-time paresthesia steering using continuous electric field adjustment. Part 1: intraoperative performance. Neuromodulation. 2004;7:157-167.

[45] Veraart C, Grill WM, Mortimer JT. Selective control of muscle activation with a multipolar cuff electrode. IEEE Trans Biomed Eng. 1993;40:640-653.

[46] North RB, Kumar K, Wallace MS, Henderson JM, Shipley J, Hernandez J, Mekel-Bobrov N, Jaax KN. Spinal cord stimulation versus re-operation in patients with failed back surgery syndrome: an 
international multicenter randomized controlled trial (EVIDENCE study). Neuromodulation. 2011;14:330-335.

[47] British Pain Society. Spinal cord stimulation for the management of pain: recommendations for best clinical practice. London: British Pain Society, 2005.

[48] Manola L, Holsheimer J. Single vs dual mode stimulation in spinal cord stimulation-what is the difference? Neuromodulation. 2006;9:150-151.

[49] Gildenberg PL. History of electrical neuromodulation for chronic pain. Pain Med. 2006;7:S7-13.

[50] Doleys D. Psychological factors in spinal cord stimulation therapy: brief review and discussion. Neurosurg Focus. 2006;21:1-6.

[51] Holsheimer J. Principles of neurostimulation. Electrical stimulation and the relief of pain. Pain research and clinical management. 2003;15:17-36.

[52] Erickson DL. Percutaneous trial of stimulation for patient selection for implantable stimulating devices. J Neurosurg. 1975;43:440-444.

[53] Hosobuchi Y, Adams JE, Weinstein PR. Preliminary percutaneous dorsal column stimulation prior to permanent implantation. J Neurosurg. 1972;17:242-245.

[54] North RB, Fischell TA, Long DM. Chronic stimulation via percutaneously inserted epidural electrodes. Neurosurgery. 2003;52:572-579.

[55] Cameron T. Safety and efficacy of spinal cord stimulation for the treatment of chronic pain: a 20year literature review. J Neurosurg. 2004;100:254-267.

[56] North RB, Kidd DA, Olin J. Spinal cord stimulation for axial low-back pain: a prospective controlled trial comparing 16-contact insulated electrodes with 4-contact percutaneous electrodes. Neuromodulation. 2006;1:56-67.

[57] Wesselink WA, Holsheimer J, King GW, Torgerson NA, Boom HBK. Quantitative aspects of the clinical performance of transverse tripolar spinal cord stimulation. Neuromodulation. 1999;2:5-14.

[58] Barolat G. Spinal cord stimulation for chronic pain management. Arch Med Res. 2000;31:258-262.

[59] Manola L, Holsheimer J, Veltink P, Bradley K and Peterson D. Theoretical investigation into longitudinal cathodal field steering in spinal cord stimulation. Neuromodulation. 2007;10: 120-132.

[60] Prager JP, Chang JH. Transverse tripolar spinal cord stimulation produced by a percutaneously placed triple lead system. Presented at the International Neuromodulation Society World Pain Congress, San Franscisco, August 2000. Abstract.

[61] White paper prepared by Medtronic 2007 Computer modeling of spinal cord stimulation for low back pain.

[62] Buvanendran A and Lubenow T J. Efficacy of transverse tripolar spinal cord stimulator for the relief of chronic low back pain from failed back surgery Pain Physician. 2008;11:333-338. 
[63] Law JD. Spinal stimulation: Statistical superiority of monophasic stimulation of narrowly separated, longitudinal bipoles having rostral cathodes. Appl. Neurophysiol. 1983;46:129-137.

[64] Coburn B. A theoretical study of epidural electrical stimulation of the spinal cord-part 2: Effect on long myelinated fibers. IEEE Trans Biomed Eng. 1985;32:978-986.

[65] Law JD. Targeting a spinal stimulator to treat failed back surgery syndrome. Appl. Neurophysiol. 1987;50:437-438.

[66] Holsheimer J, Nuttin B, King GW, Wesselink WA, Gybels JM, de Slutter P. Clinical evaluation of paresthesia steering with a new system for spinal cord stimulation. Neurosurgery. 1998;42:541-549.

[67] Barolat G, Massaro F, He J, Zeme S and Ketcik B. Mapping of sensory responses to epidural stimulation of the intraspinal neural structures in man. J Neurosurg. 1993;78:233-239.

[68] Linderoth B. Spinal cord stimulation in ischemia and ischemic pain. Possible mechanisms of action. In: Horsch S, Claeys L (eds) Spinal cord stimulation 2: an innovative method in the treatment of PVD and angina. Steinkopff, Darmstadt; Springer, Berlin, Darmstadt. 1995;19-35.

[69] Coburn B. Electrical stimulation of the spinal cord: two-dimensional finite element analysis with particular reference to epidural electrodes. Med Biol Eng Comput. 1980;18:573-584.

[70] Sin WK, Coburn B. Electrical stimulation of the spinal cord: a further analysis relating to anatomical factors and tissue properties. Med Biol Eng Comput. 1983;21:264-269.

[71] Struijk JJ, Holsheimer J, van Veen BK, Boom HBK. Epidural spinal cord stimulation: calculation of field potentials with special reference to dorsal column nerve fibers. IEEE Trans Biomed Eng. 1991;38:104-110.

[72] Holsheimer J, Den Boer JA, Struijk JJ, Rozeboom AR. MR assessment of the normal position of the spinal cord in the spinal canal. Am J Neuroradiol. 1994;15:951-959.

[73] McNeal DR. Analysis of a model for excitation of myelinated nerve. IEEE Trans Biomed Eng. 1976;23:329-337.

[74] Wesselink WA, Holsheimer J, King GW, Torgerson NA, Boom HBK. Quantitative aspects of the clinical performance of transverse tripolar spinal cord stimulation. Neuromodulation. 1999; 2:5-14.

[75] Holsheimer J. Computer modeling of spinal cord stimulation and its contribution to therapeutic efficacy (Review) Spinal cord. 1998;36:531-40.

[76] McCreery DB, Agnew WF, Yuen TG, Bullara L. Charge density and charge per phase as cofactors in neural injury induced by electrical stimulation. IEEE Trans Biomed Eng. 1990;37:996-1001. 
Chapter 1 


\title{
CHAPTER 2
}

\section{Triple leads programmed to perform as longitudinal guarded cathodes in SCS - a modeling study}

\author{
Vishwanath Sankarasubramanian, Jan. R. Buitenweg, Jan Holsheimer, Peter Veltink \\ MIRA, Institute for Biomedical Technology and Technical Medicine, University of Twente, Enschede, \\ The Netherlands
}

Published in Neuromodulation, 14(5):401-411, August 2011 


\begin{abstract}
Objective: In spinal cord stimulation, neurosurgeons increasingly tend to implant dual leads. Dual leads (longitudinal bipole/tripole) provide medio-lateral control over the recruited dorsal column (DC) area by steering the injected cathodal currents. However the DC recruited area is suboptimal when dual aligned leads straddling the midline programmed as longitudinal guarded cathodes (+-+) are used instead of a single lead placed over the spinal cord midline with the same configuration. As a potential improvement, an additional third lead between the two aligned leads is modeled to maximize the medio-lateral extent of the recruited DC area at the low-thoracic vertebral region (T10-12).
\end{abstract}

Methods and materials: The University of Twente Spinal Cord Stimulation software (UT-SCS) is used in this modeling study. Longitudinal guarded cathodes were modeled on the low-thoracic vertebral region (T10-T12) using percutaneous triple lead configurations. The central lead was modeled over the spinal cord midline and the two lateral leads were modeled at several transverse distances to the midline lead. Medio-lateral field steering was performed with the midline lead and the second lead on each side to achieve constant anodal current ratios (CAR) and variable anodal current ratios (VAR).

Results: Reducing the transverse lead separation resulted in increasing the depths and widths of the recruited DC area. The triple lead configuration with the least transverse separation had the largest DC recruited area and usage range. The maximum DC recruited area (in terms of both depth and width) was always found to be larger under VAR than CAR conditions.

Conclusions: Triple leads programmed to perform as longitudinal guarded cathodes provide more postoperative flexibility than single and dual leads in covering a larger width of the low-thoracic DCs. The transverse separation between the leads is a major determinant of the area and distribution of paresthesia. 


\subsection{Introduction}

Spinal cord stimulation (SCS) is a clinically established neuromodulation technique increasingly used in the treatment of chronic, intractable pain. It is based on the "gate control" concept of electrical activation of pain-inhibiting neuronal mechanisms. The clinical manifestation of SCS is the induction of a tingling sensation called paresthesia which should cover the complete pain area (1-3). Such a paresthetic sensation can be evoked by stimulation of both dorsal column (DC) and dorsal root (DR) fibers, being part of the same large cutaneous afferent fibers (4-6). However, the paresthesia coverage will differ strongly when either DCs or DRs are stimulated. Generally, DC fibers are targeted as their somatotopic organization allows a broader area of paresthesia. DR fibers, on the other hand, evoke paresthesia only in 1-2 dermatomes (5). The differential activation of DC and DR fibers depends on several factors: anodecathode combination, the longitudinal distance between cathodes and anodes, distance between the posterior aspect of the spinal cord and the epidural lead (also defined as the thickness of the dorsal cerebrospinal fluid layer, dCSF) and the conductivity difference of the CSF and white matter at their interface $(4,6)$. DR fibers are preferentially excited by monopolar stimulation (4). Longitudinal guarded cathode $(+-+)$ and bipolar stimulation are preferred due to the increased activation of DC fibers, but only if the contact distance and dCSF do not exceed 3-4 mm (7). When comparing bipolar with guarded cathode stimulation, computer modeling predicts that the latter would yield even better DC activation (8). Modeling studies so far predict that maximum DC activation is achieved with a single longitudinal guarded cathode (also called a longitudinal tripole) placed over the spinal cord midline, and having a small contact center distance and a small dCSF $(4,9)$. Single percutaneous leads, however, pose the threat of suboptimal lead placement and lead migration. The latter often results in a change in paresthesia location which may require additional surgical intervention. A solution that may reduce the need for additional surgery, either in the case of migration or suboptimal placement, is stimulation by two aligned leads, each programmed as a longitudinal bipole or guarded cathode; this is termed "dual lead stimulation”. This provides medio-lateral control over the activated DC area by steering the injected cathodal currents. However, modeling studies show that a DC area of smaller medio-lateral size than a single lead combination is activated (9). In a clinical study by North et al. 2005 (10), it was also shown that two leads positioned at opposite sides of the DC midline yields a lower paresthesia coverage than a single percutaneous lead positioned over the physiological midline. Since the DC recruited area was suboptimal when two aligned leads straddling the spinal cord midline were used, an additional third lead placed between the two aligned leads might be useful to cover the full lateral extent of the DCs at the lowthoracic vertebral region (T10-12), which is about $5 \mathrm{~mm}$ wide (11). The first reported use of three percutaneous leads was in 1983 where Jay Law implanted patients with three parallel, multi-contact leads 
to optimize the stimulation field to achieve the best possible paresthesia coverage for low-back pain $(12,13)$.

The aim of this modeling study is to maximize the low-thoracic DC coverage using triple leads programmed as longitudinal guarded cathodes, with the center lead over the spinal cord (SC) midline. Although three leads are inserted within the dorsal epidural space, only two out of the three leads are chosen simultaneously for stimulation. Medio-lateral field steering was performed using the midline lead and the second lead on each side by varying the proportion of cathodal currents. The anodal currents were either kept constant or were varied proportionally with the cathodal currents through the respective leads to analyze whether simultaneous anodal steering increases the effect of cathodal steering on the recruitment of DC fibers. The transverse separation between the leads is varied to study the effect on the usage range and the maximum recruited DC area (in terms of both depth and width).

\subsection{Methods}

The University of Twente Spinal Cord Stimulation software (UT-SCS) is used in this modeling study. This software permits the implementation of a three-dimensional volume conductor model of the spinal column, including electrode arrays and nerve fibers.

\subsubsection{Volume conductor model}

A 3D model of the low-thoracic vertebral region (T10-T12) was used. Its transverse geometry is shown in Figure 1. The electrical conductivities of the human anatomical structures from earlier modeling studies (6) were used, except for the values of the dura mater and the surrounding layer, which were adjusted to match recent lead contact impedance data (14). The conductivities of the tissues in the volume conductor model are shown in Table 1 . The total dimensions of the model were $24.1 * 25.7 * 59.35 \mathrm{~mm}$ divided into $64 * 64 * 80$ non-equidistant cubic elements in the medio-lateral, dorso-ventral and rostro-caudal direction respectively. The dCSF was set at $3.2 \mathrm{~mm}$. Current was injected into the model by means of cathodal and anodal contacts on two of the three percutaneous leads positioned in the dorsal epidural space of the model, adjacent to the dura mater.

To calculate the stationary potential field for each element, Ohms law is used:

$$
-\sigma \cdot \vec{\nabla} V=\vec{J}
$$

Where $\mathrm{J}$ is the current density $\left(\mathrm{A} / \mathrm{m}^{2}\right), \sigma$ is the conductivity of the material $(\mathrm{S} / \mathrm{m})$ and $\mathrm{V}$ is the potential (V). This formula can also be presented as Poisson's equation for a conductive medium: 


$$
\vec{\nabla} \cdot(\sigma \vec{\nabla} \cdot V)=-\vec{\nabla} \cdot \vec{J}
$$

With this formula it is possible to calculate the current density for every point in a 3D space, where $\mathrm{J}$ would be the current density at the point $(\mathrm{x}, \mathrm{y}, \mathrm{z}) . \sigma$ and $\mathrm{V}$ are scalars, $\mathrm{J}$ is a vector. All conductivities are isotropic except for that of the white matter. The conductivity of white matter is highest in the $\mathrm{z}$ direction, while in the $\mathrm{x}$ and $\mathrm{y}$ directions the conductivity is lower and isotropic (6).

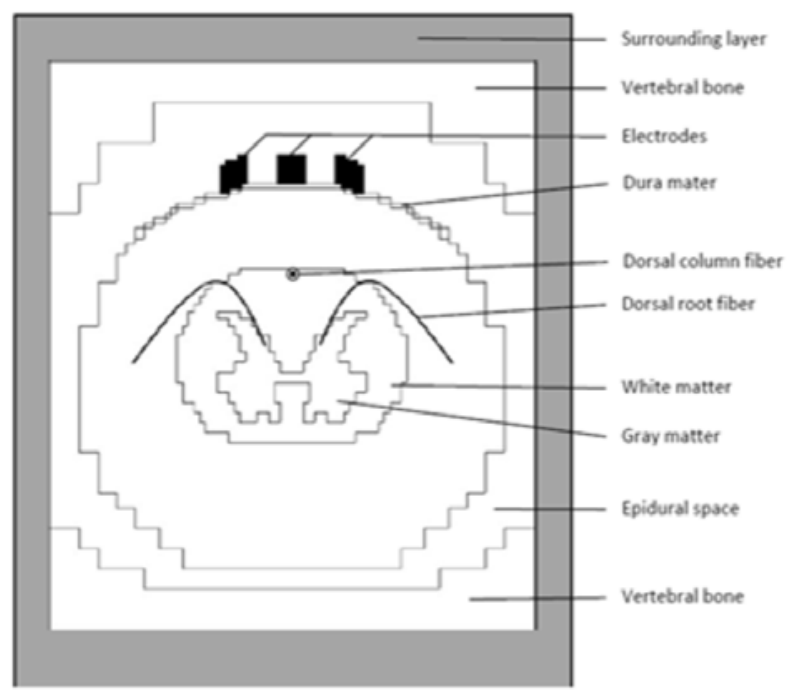

Figure 1 Transverse section of the low-thoracic UT-SCS model of the spinal cord with the volume conductor elements, electrodes and the nerve fibers. The grids are not depicted.

Before a unique solution can be calculated for equation (2.1), boundary conditions need to be set. A zero potential layer is defined around the spinal column model (the surrounding layer) representing distant body tissue. At the location of the electrodes the predefined stimulation potentials or currents are defined. Defined values for boundaries of an area or plane are known as Dirichlet boundary conditions.

At the edges of the entire volume, Neumann border conditions are defined. This condition states that the normal derivative component of a surface is zero. In this case the normal component of a surface would be the flowing current. Stating that the normal component at the outer border of the volume is zero means there is no current flowing outside the volume. To calculate the current densities throughout the entire volume a finite element method is used to apply formula 2.1 to the specific grid. This way a large set of linear equations is created which can be used to calculate the current densities inside the entire volume. This set of linear equations can be solved using a Red-Black Gauss-Seidel numerical method with a variable over-relaxation factor. 
Table 1 Conductivities of the tissues in the volume conductor model

\begin{tabular}{cc}
\hline Tissue & $\boldsymbol{\sigma}(\mathbf{S} / \mathbf{m})$ \\
\hline CSF & 1.70 \\
Dura mater & 0.60 \\
Electrode insulation & 0.001 \\
Epidural space & 0.04 \\
Gray mater & 0.23 \\
Vertebral bone & 0.02 \\
White mater: & \\
Transverse & 0.083 \\
Longitudinal & 0.60 \\
\hline
\end{tabular}

\subsubsection{Lead-type and transverse separation}

The influence of the transverse lead separation was evaluated by modeling three percutaneous leads on the low-thoracic vertebral region (T10-T12) with different lateral lead separations (Fig. 2). The percutaneous leads (model Advanced Bionics AB SC2108) have eight contacts each. The cylindrical contacts with a diameter of $1.35 \mathrm{~mm}$ were modeled as four rectangular surfaces around an insulating square bar. The contact width and length is $1.0 \mathrm{~mm}$ and $3.0 \mathrm{~mm}$ respectively. The total surface area of all four contact surfaces is $4 * 3 \mathrm{~mm} * 1 \mathrm{~mm}=12.0 \mathrm{~mm}^{2}$. The longitudinal edge-to-edge spacing between the contacts was $1.0 \mathrm{~mm}$. Tripolar longitudinal guarded cathode (+-+) combinations on adjacent contacts were modeled using these leads. Hence, only three out of the eight contacts are used for stimulation. The three percutaneous leads are aligned with each other and there are in total nine aligned active contacts. The central lead is placed in the symmetry plane of the spinal column model. The current model assumes the anatomical (vertebral) and physiological (spinal cord) midline to be the same. The lateral leads are not placed more than $3 \mathrm{~mm}$ apart from the midline lead so that current steering between the leads may occur. Also, the leads are not placed less than $1 \mathrm{~mm}$ apart as surgical feasibility of such configurations is remote. The transverse separations between the midline and the lateral leads were chosen to be 1.0, 1.5, 2.0 and 2.5 mm edge-to-edge (Fig. 2). 


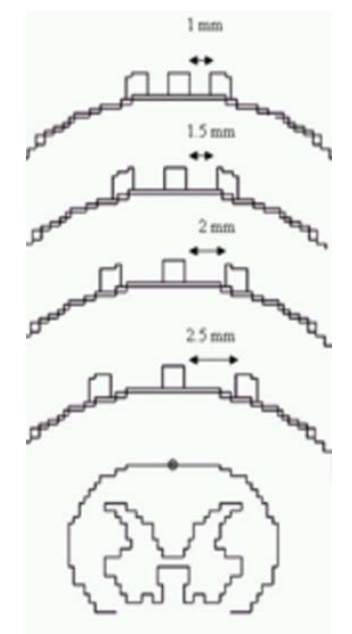

Figure 2 Transverse cross-section of the model showing the relative lead position and the edge-to-edge spacing of the corresponding contacts.

\subsubsection{Stimulation parameters}

Current-controlled electrical field steering was performed with two out of three leads simultaneously; in this case with the left (lateral) and the midline lead (Fig. 3). Current steering with the right (lateral) and the midline lead is symmetrical to that of the left and the midline lead. The authors arbitrarily assumed the pulse width to be $210 \mu \mathrm{sec}$, since in clinical studies the average pulse width ranges from 175-600 $\mu$ sec (15). Cathodal currents were varied at cathodal current ratios (CCRs) of 100, 90, 80, 70, 60, 50, 40, 30, 20, 10 and $0 \%$ (ratios of $10 \%$ ). CCR is defined as the percentage of current applied to the midline cathode. Simulations were performed at both a constant anodal current ratio (CAR; since there are 4 active anodes in 2 leads, each anode received 25\% of the total cathodal current through both leads) and a variable anodal current ratio (VAR; since there are 2 active anodes in each lead, each anode received 50\% of the cathodal current through the same lead). 

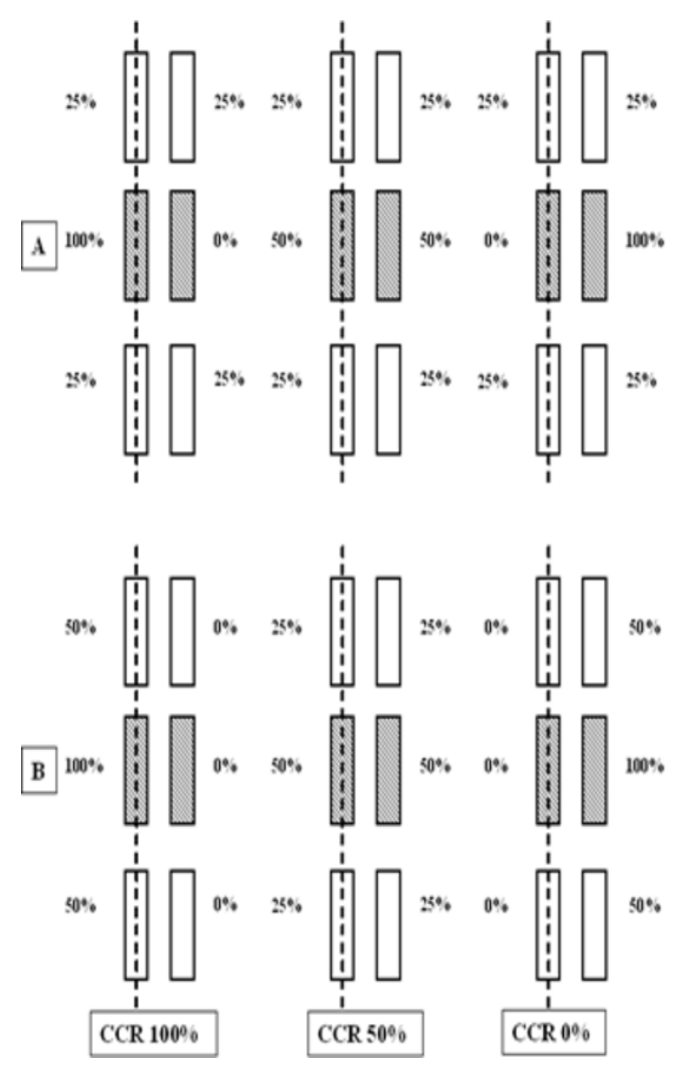

Figure 3 Schematic representation of electrical current steering mechanisms. (A) A total cathodal current of $100 \%$ is varied between the right and the left cathode at CCRs of 100, 50 and 0\%. The anodal current is kept constant at $25 \%$ of the total cathodal current (CAR) for each of the 4 anodes. (B) A total cathodal current of $100 \%$ is varied between the right and the left cathode at various CCRs of 100, 50 and 0\%. The anodal current is also varied proportionally along with the cathodal current in the same lead, in which case the current is balanced per lead (VAR). The cathodes are hatched.

\subsubsection{Nerve fiber model}

In 1976, McNeal developed a mathematical model of a myelinated fiber, based on the electrical cable network (16). For the description of the nodal membrane kinetics during excitation, in particular to determine the ionic current at each node of Ranvier, McNeal used the Frankenhaeuser-Huxley equations that had been derived in 1964 for frog myelinated nerve fibers (17). Furthermore, an extension of McNeal's fiber model for fibers with collaterals was considered, as described by Struijk et al (18). The values of the parameters of these equations were later modified by Wesselink et al. (19) in order to make them correspond better to human data. This modified version of the fiber model is implemented in this work. The parameters of the fiber membrane are mentioned in Table 2. 
Table 2 Fiber model and its parameters

\begin{tabular}{lll}
\hline Parameter & Symbol & Value \\
\hline Membrane capacity & $\mathrm{C}_{\mathrm{m}}$ & $0.028 \mathrm{~F} / \mathrm{m}^{2}$ \\
Leakage conductance & $\mathrm{g}_{\mathrm{L}}$ & $600 \mathrm{~S} / \mathrm{m}^{2}$ \\
Sodium permeability & $\mathrm{p}_{\mathrm{Na}}$ & $0.0704 \mathrm{dm}^{3} / \mathrm{m}^{2} *_{\mathrm{S}}$ \\
Potassium conductance & $\mathrm{g}_{\mathrm{K}}$ & $300 \mathrm{~S} / \mathrm{m}^{2}$ \\
Intra-axonal resistance & $\rho_{\mathrm{a}}$ & $0.33 \Omega \mathrm{m}$ \\
Leakage equilibrium potential & $\mathrm{V}_{\mathrm{L}}$ & $-84.14 \mathrm{mV}$ \\
Sodium equilibrium potential & $\mathrm{V}_{\mathrm{Na}}$ & $43.7 \mathrm{mV}$ \\
Sodium concentration outside cell & $\mathrm{Na}_{\mathrm{O}}$ & $154 \mathrm{mM}$ \\
Sodium concentration inside cell & $\mathrm{Na}_{\mathrm{i}}$ & $30 \mathrm{mM}$ \\
Potassium equilibrium potential & $\mathrm{V}_{\mathrm{K}}$ & $-84 \mathrm{mV}$ \\
Resting membrane potential & $\mathrm{V}_{\mathrm{r}}$ & $-84 \mathrm{mV}$ \\
Faraday constant & $\mathrm{F}$ & $96485 \mathrm{C} / \mathrm{mole}$ \\
Gas constant & $\mathrm{R}$ & $8.3144 \mathrm{~J} / \mathrm{K} * \mathrm{~mole}$ \\
Absolute temparature & $\mathrm{T}$ & $310.15 \mathrm{~K}$ \\
\hline
\end{tabular}

In the UT-SCS model several types of nerve fibers can be defined. The nerve fibers used in this study had the following standard diameters:

DC fibers (without collaterals): $12 \mu \mathrm{m}$ in the median $66 \%$ of the DCs and linearly increasing to $15 \mu \mathrm{m}$ at the lateral borders, thus mimicking the lower fiber threshold due to the presence of an increasing density of collaterals near the bifurcation of the corresponding DR fibers $(18,20)$.

DR fibers: $15 \mu \mathrm{m}$, with an ascending and a descending $12 \mu \mathrm{m}$ DC fiber with collaterals attached. Since a low-thoracic segment was modeled, we chose to model the 'type A1' DR fiber, as described by Struijk et

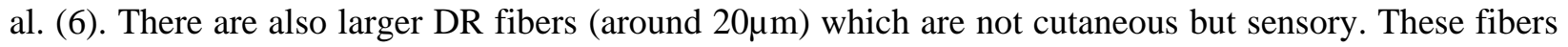
have other functions, such as in reflex movements, and are not involved in the perception of paresthesia. Therefore, these fibers were not modeled and simulated.

The DC fiber diameter is assumed to be the same independent of fiber depth. Fiber thresholds were computed at the minimal cathodal current required to elicit an action potential. The modeled DC fiber was shifted medio-laterally along the dorsal surface of the DCs to identify the minimum activation threshold. 


\subsubsection{Model output parameters}

The combination of the stimulus-induced potential field and the nerve fiber model enabled the calculation of several clinically relevant SCS output parameters, such as paresthesia and discomfort thresholds (PT and DT), usage range, and maximum recruited DC area. A complete list of parameters is presented in Table 3.

Table 3 Model output parameters

\begin{tabular}{lcl}
\hline \multicolumn{1}{c}{ Parameter } & Unit & \multicolumn{1}{c}{ Description } \\
\hline $\begin{array}{l}\text { DC fiber threshold } \\
\left(\mathrm{I}_{\mathrm{DC}}\right)\end{array}$ & $\mathrm{mA}$ & Lowest activation threshold among all DC fibers \\
$\begin{array}{l}\text { DR fiber threshold } \\
\left(\mathrm{I}_{\mathrm{DR}}\right)\end{array}$ & $\mathrm{mA}$ & $\begin{array}{l}\text { Lowest activation threshold of the DR fiber. Calculated as the } \\
\text { minimum of } \mathrm{I}_{\mathrm{DR}, \mathrm{L}} \text { and } \mathrm{I}_{\mathrm{DR}, \mathrm{R}}\end{array}$ \\
$\begin{array}{l}\text { Paresthesia } \\
\text { threshold (PT) }\end{array}$ & $\mathrm{mA}$ & $\begin{array}{l}\text { Current required to activate the lowest threshold fiber, being either } \\
\text { a DC or DR fiber. Lowest value between } \mathrm{I}_{\mathrm{DC}} \text { and } \mathrm{I}_{\mathrm{DR}}\end{array}$ \\
$\begin{array}{l}\text { Discomfort } \\
\text { threshold (DT) }\end{array}$ & $\mathrm{mA}$ & $\begin{array}{l}\text { Current at initial stimulation of proprioceptive DR fibers, defined } \\
\text { as } 1.4^{*} \mathrm{I}_{\mathrm{DR}}(20)\end{array}$ \\
$\begin{array}{l}\text { Usage range } \\
\text { Ratio of discomfort and paresthesia threshold. }\end{array}$ \\
$\begin{array}{l}\text { Maximum DC } \\
\text { recruited area }\end{array}$ \\
$\begin{array}{l}\text { Width } \\
\text { mm }\end{array}$
\end{tabular}

\subsection{Results}

\subsubsection{Constant and variable anodal current ratios}

$\mathrm{I}_{\mathrm{DC}}$ and $\mathrm{I}_{\mathrm{DR}}$, usage range and the maximum DC recruited area (including depth and width of the recruited area) were determined for VAR as a function of CCR and compared with the respective values of CAR. In this comparative study, the leads were separated by $2.5 \mathrm{~mm}$ edge-to-edge (Fig. 4).

Under VAR conditions, $\mathrm{I}_{\mathrm{DC}}$ decreased while $\mathrm{I}_{\mathrm{DR}}$ increased from the left to the midline lead at all CCR values (Fig. 4). The decrease of $I_{D C}$ was small (less than 10\%) compared to the increase of $I_{D R}$. $I_{D R}$ and usage range showed an increase up to $50 \%$ at some CCR values. The maximum $I_{D R}(9.49 \mathrm{~mA})$ and the 
maximum UR (3.97) were present at 100\% CCR (represents the case of a single lead with longitudinal guarded cathode placed on the spinal cord midline). The largest DC recruited area was also obtained at 100\% CCR (Fig. 4).
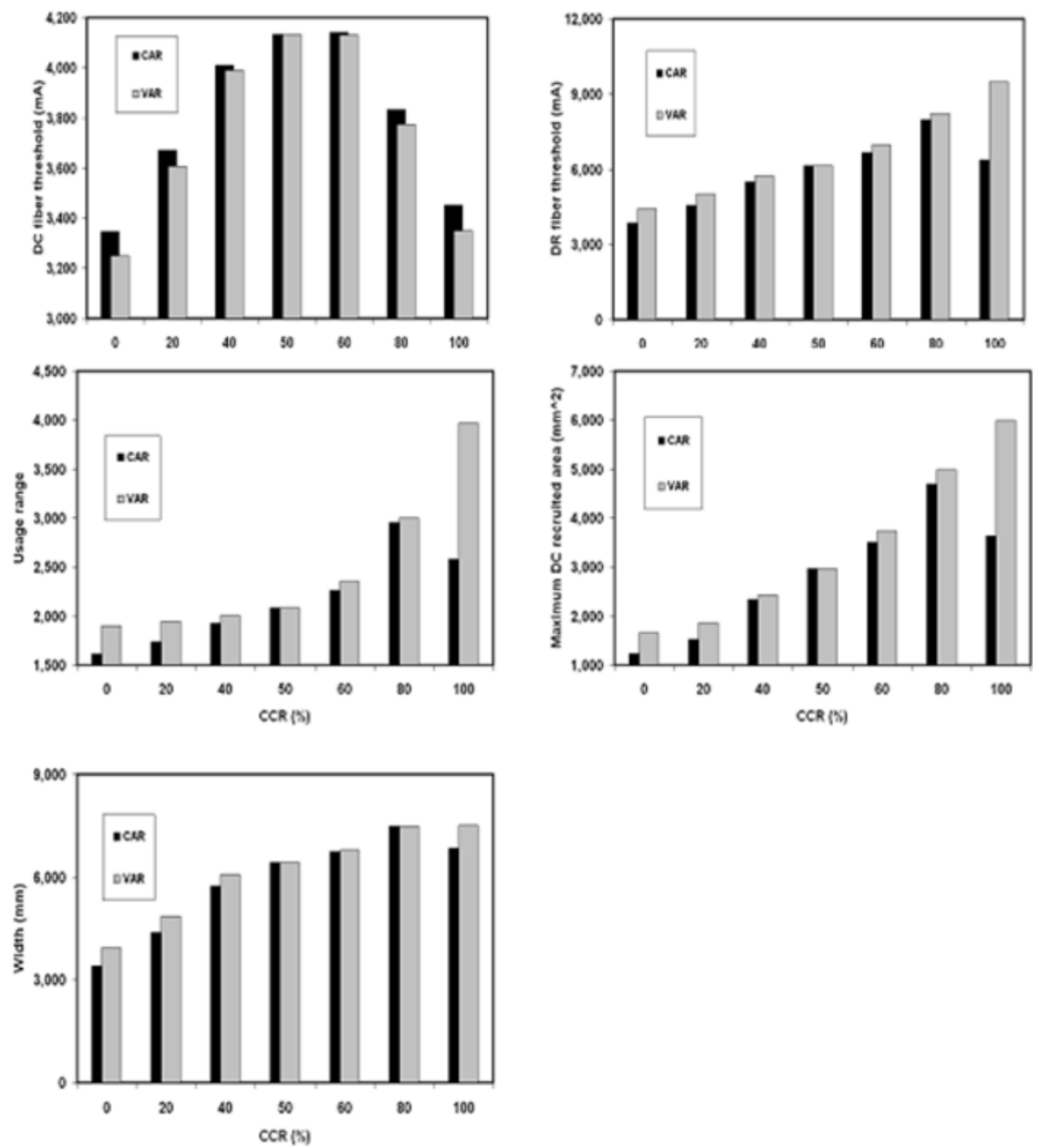

Figure 4 Output parameters ( $I_{D C}, I_{D R}$, usage range, maximum DC recruited area, width) for VAR and CAR at a transverse lead separation of $2.5 \mathrm{~mm}$ edge-to-edge. The output parameters for VAR (except DC fiber threshold, $\left.I_{D C}\right)$ are larger than with CAR at all CCR values. The maximum difference between their values occurs at 100\% CCR. Note: CCR of 0\% denotes that all cathodal current is on the lateral lead and $100 \%$ denote that all cathodal current is on the midline lead. 


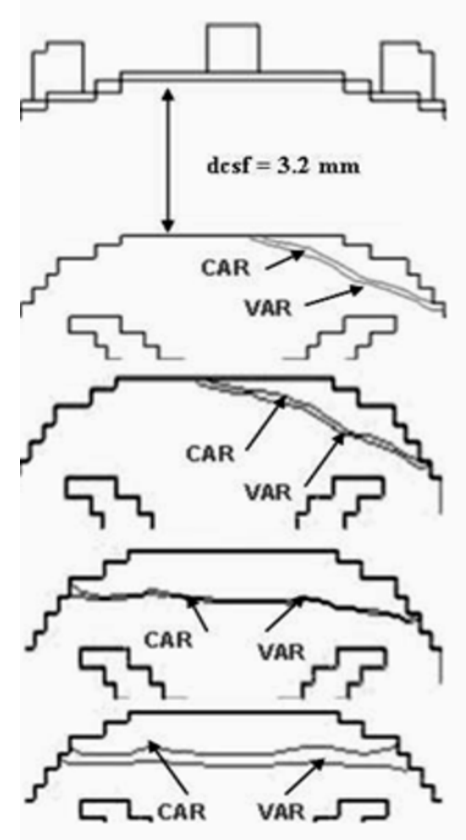

Figure 5 Recruited DC areas for VAR and CAR at 0\% (top), 20\%, 80\% and 100\% (bottom) CCRs. The differences between the recruited areas are largest at 100\% CCR. A prominent difference is also observed at $0 \%$ CCR.

The contours of the maximum DC recruited area are compared in more detail in Figure 5. The recruitment contours, indicating the ventral boundary of the DC recruited area were compared for both CAR and VAR conditions and at CCRs of 0, 20, 80 and 100\%. It was shown that the maximum DC recruited area (both depth and width of the DC recruited area) is larger under VAR than CAR conditions at all CCR values. However, these differences were largest at 100\% CCR.

Since the above results show that the maximum DC recruited area is always larger under VAR than under CAR conditions, the results below are presented only for VAR.

\subsubsection{Effect of transverse lead separation on fiber thresholds, usage range and DC recruitment}

Paresthesia and discomfort: The effects of transverse lead separation on the paresthesia and discomfort thresholds (PT and DT) as a function of CCR are shown in Figure 6. The lowest activation threshold of the DC and DR fibers was defined as paresthesia threshold (9). PT had its minimum value when the entire current was applied to one of the cathodes (medial or lateral) and reached a maximum value when the total cathodal current was equally divided among the two cathodes. 


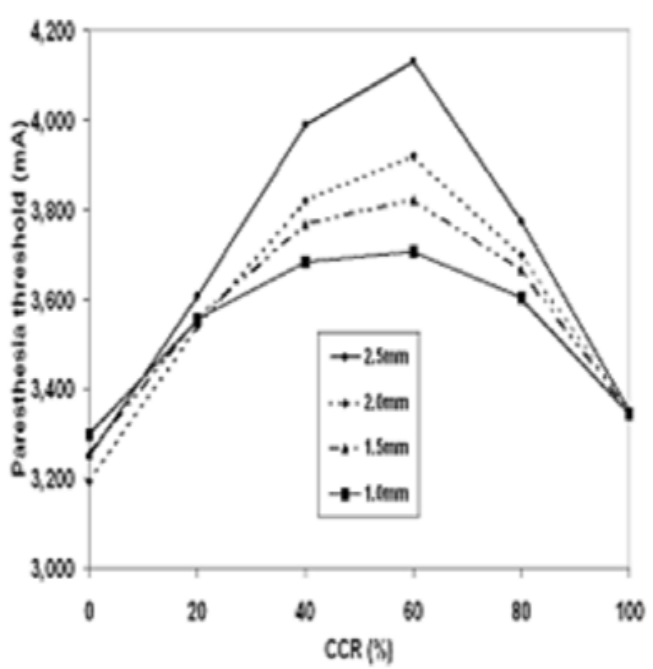

(a)

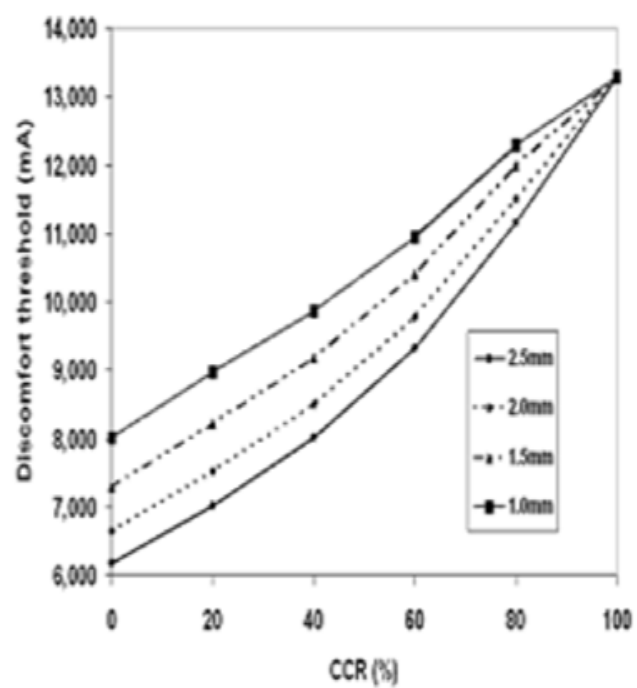

(b)

Figure 6 Variation of (a) Paresthesia threshold (PT) and (b) Discomfort threshold (DT) for VAR as a function of CCR at various transverse lead separations of 1, 1.5, 2.0 and $2.5 \mathrm{~mm}$.

The threshold current of the fibers in the DRs associated with discomfort is termed discomfort threshold (DT); numerically, it is defined as $1.4 * \mathrm{I}_{\mathrm{DR}}(21)$. DT had a minimum value when the entire current was applied to the lateral cathode and reached a maximum value when the entire current was passed through to the midline cathode.

PT showed a decrease when the transverse lead separations were decreased. The DT showed an increase. The transverse lead separation thus had a contrasting effect on the fiber thresholds. It can also be seen that for larger lead separations, the CCR has a greater effect on the PT and DT. 
Usage range and DC recruitment: The effect of transverse lead separation on usage range and maximum DC recruited area as a function of CCR is shown in Figure 7.

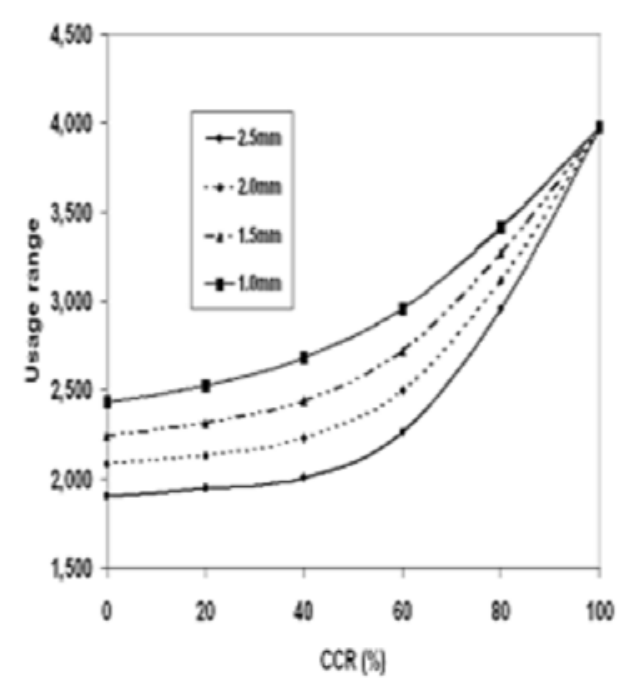

(a)

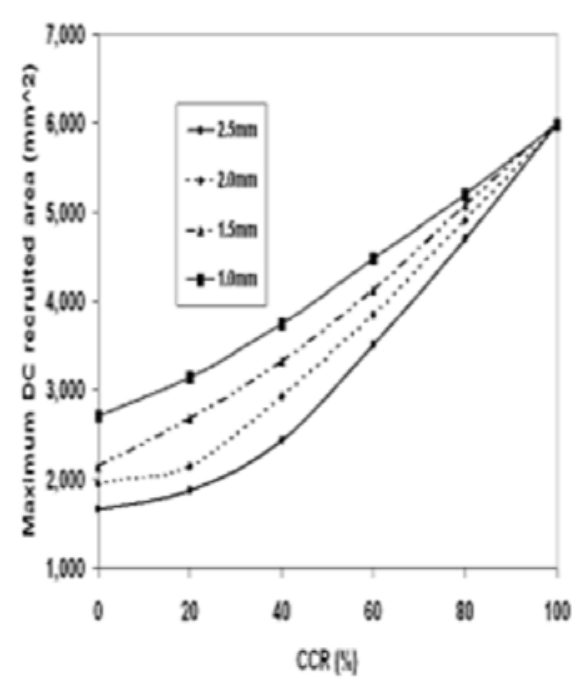

(b)

Figure 7 Variation of (a) Usage range and (b) Maximum DC recruited area for VAR as a function of CCR at various transverse lead separations of 1, 1.5, 2.0 and $2.5 \mathrm{~mm}$.

Both, maximum DC recruited area and usage range varied in a similar pattern: for CCRs, from 0 to $100 \%$. They reached their minimum when the entire current was applied to the lateral cathode and their maximum when the entire current was applied to the medial cathode. The contours of the maximum DC recruited areas are shown from top to bottom in Figure 8 for each transverse lead separation. Indicated from left to right in the same figure are the DC recruitment contours at CCRs of 0, 50 and 100\%. With the CCR varying from the lateral (0\%) to the medial cathode (100\%), the recruited DC area - initially located on the lateral (left) side - extended to the contra-lateral side and became more symmetrical. When the transverse separation between the leads was smaller $(2.0 \mathrm{~mm}$ to $1.5 \mathrm{~mm}$ to $1.0 \mathrm{~mm})$, the DC recruited area (both depth and width) became both larger and less variable (Fig. 8).

The triple lead configuration with $1 \mathrm{~mm}$ transverse separation between the leads had the maximum and least varying $S_{\mathrm{RA}}$ and UR across the whole range of CCRs. 

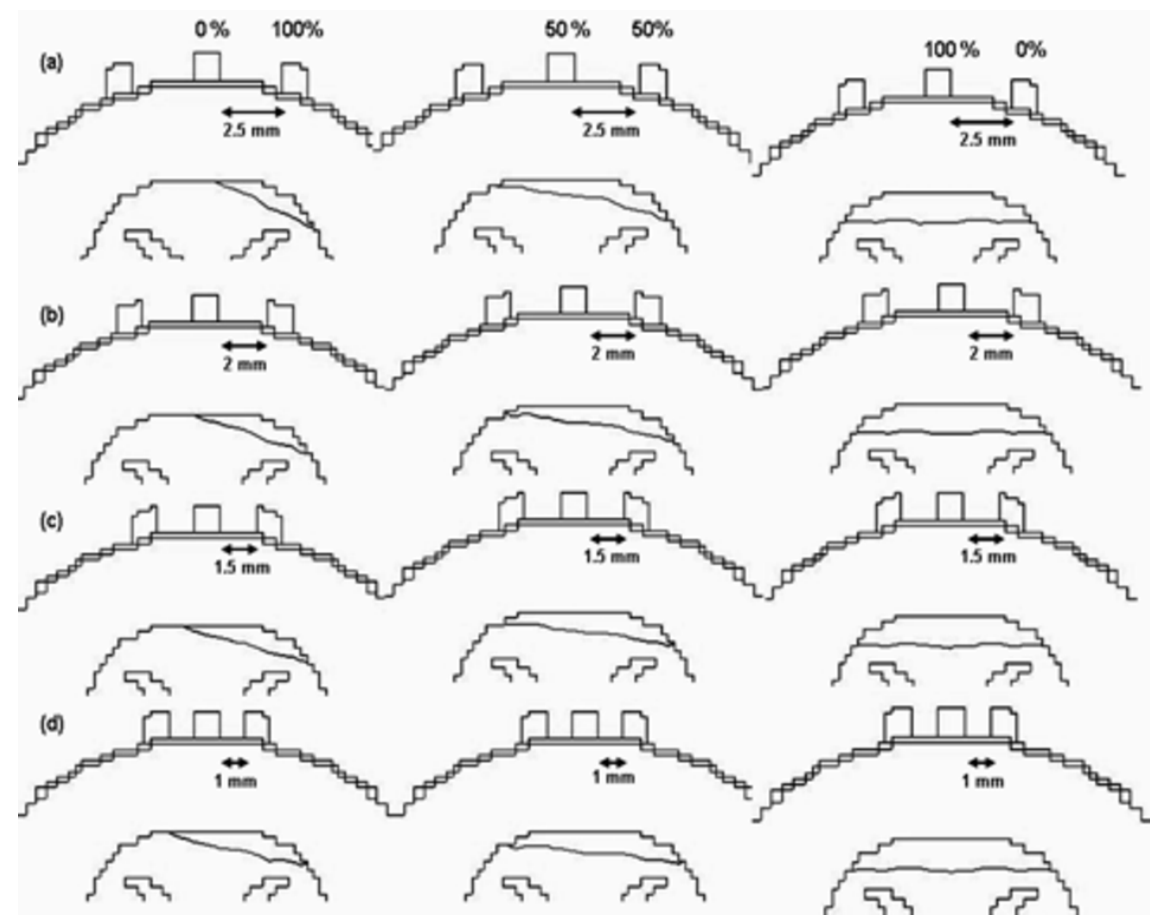

Figure 8 Maximum DC recruitment contours $\left(S_{R A}\right)$ for VAR at lead separations of (a) $2.5 \mathrm{~mm}$, (b) $2 \mathrm{~mm}$, (c) $1.5 \mathrm{~mm}$ and (d) $1 \mathrm{~mm}$; for each lead separation, the maximum DC recruited area is shown as the CCR is altered from $0 \%$ (smallest DC area) to $100 \%$ (largest DC area).

\subsection{Discussion}

The primary aim of this modeling study was to simulate the conditions in SCS, to maximize the recruitment of the low-thoracic DCs and paresthesia in the corresponding dermatomes using triple leads programmed as longitudinal guarded cathodes (+-+).

\subsubsection{Triple leads versus dual leads}

Clinical studies have shown both improved $(22)$ and worse $(23,24)$ performances of dual leads in comparison to single leads. In previous modeling studies into dual-lead longitudinal guarded cathodes positioned symmetrically to the spinal cord midline, cathodal steering capabilities were demonstrated, but these came at the cost of a reduced recruited area and width of the DCs compared to a midline lead (9). Moreover, with dual leads straddling the spinal cord midline, the DC recruitment was always bilateral if the current was evenly distributed in both the leads. This is where simulation with the third lead placed on the spinal cord midline might prove to be beneficial in covering the full lateral extent of the DCs at lowthoracic vertebral regions. Also, it should be noted that by inserting triple leads, there is always an option and hence a potential advantage of any 2 leads being chosen for stimulation (either the 2 lateral leads, or 
the midline and one of the lateral leads). The choice can be made based on the optimal programming parameters and pain condition of the patient.

The current modeling study with triple leads has involved stimulation with the midline lead and the second lead on each side. The results showed that medio-lateral current steering between the left and the midline lead extended the recruitment of DCs from being completely in the left DCs to equally distributed in both the DCs, until the recruited DC area was maximized (Fig. 8). Similarly, current steering between the right and the midline lead would extend the recruitment of DCs from being completely in right DCs to equally distribute in both the DCs. There is a probability that the center lead is unable to be placed on the spinal cord midline or lateral lead migration displaces the center lead to one of the sides. In this case, there is still the option of stimulation with the lead on the opposite side of the spinal column. This can greatly help in correcting the unilateral DC recruited area and thereby the paresthesias (9). These DC recruited areas are of course less than that of a single lead longitudinal guarded cathode placed on the spinal cord midline. However, triple leads are desirable because of their ability in coping with mediolateral displacement of the leads and providing greater post-operative flexibility than single and dual leads, in modifying the stimulation-induced electrical field. This enables multiple dermatomes to be activated which is considered to be beneficial in the reduction of widespread or complex pain.

This modeling study did not take into account the effect of lead insertion on the volume of epidural space. The anticipated volume increase may push the dura mater anteriorly and thereby reduce dCSF. In this way, triple leads may reduce the distance between the leads and the spinal cord more than single and dual leads (7).

\subsubsection{Longitudinal guarded cathodes versus transverse tripoles}

Both with dual leads placed symmetrical (the 2 lateral leads) and asymmetrical (midline lead and one of the lateral leads) to the spinal cord midline (Fig. 8), medio-lateral current steering extended the recruitment of DCs until the recruited DC area was maximized. According to the topography of the DCs, maximized medio-lateral recruitment would achieve the broadest paresthesia coverage. If the DC recruitment were clinically significant, it would imply initiation of paresthesia in a particular area (dermatomes associated with the left or the right DCs), extend to the adjacent dermatomes (corresponding to the adjacent part of DCs) until the maximum coverage is reached. Complex pain complaints, such as the ones of patients who have pain in the low back and in one or both lower extremities, require a high degree of flexibility in the implanted SCS configuration. The configuration must electronically steer the current in the medio-lateral direction and to activate multiple electrical contacts simultaneously. These goals can be best met by a system that allows triple leads programmed to perform as longitudinal guarded cathodes. 
The concept of longitudinal guarded cathodes differs basically from the Transverse tripolar stimulation concept, in that, the guarding effect of the longitudinal tripoles is predominant over anodal shielding effect of the lateral anodes. In transverse tripolar stimulation, introduced in 1996, by Struijk and Holsheimer, steering was done with 3 contacts simultaneously. As steering progresses from one side of the DCs to the other, paresthesias shift gradually in a limited number of dermatomes to the other side of the body instead of extending to the other side, as with dual leads (25-27). Also, as current steering progresses beyond a certain point, paresthesia would get lost in the dermatomes on the contra lateral side. Continued steering from one side of the body to another would mean loss of recruitment of the most lateral parts of the opposite DC. Hence, the transverse guarded cathode can be useful only in areas where the sweet spot is narrow, such as low-back pain, and stimulation outside the sweet spot results in stimulation of unwanted structures (28).

\subsubsection{Transverse lead separation}

Our modeling results showed that the transverse lead separation has a predominant effect on the nerve fiber thresholds. The authors simulated only the largest cutaneous afferent fiber parts (either DC or DR fibers). It is therefore assumed that the paresthesia threshold, PT is immediately related to the activation of those first cutaneous fibers (either DC or DR fibers) having the lowest threshold (9). PT decreased when the leads were closer together (Fig. 6a). A smaller separation between the leads causes the site of DC stimulation to be determined by the superposition of fields induced by the two leads. A smaller lead separation results in an increase of the electric field due to superposition and a more effective stimulation of the DCs. DT was increased when the separation between the leads decreased (Fig. 6b). This was expected because at reduced spacing, the most lateral lead moves away from the DR on that side, resulting in an increase of $\mathrm{I}_{\mathrm{DR}}$ on that side and hence on $D T$.

Usage range, defined as the ratio of DT and PT, was also substantially affected by the lead separation (Fig. 7). PT did not change much in comparison with DT. Hence, usage range was strongly influenced by DT. A smaller transverse lead separation, which resulted in a larger DT, provided the largest usage range. A larger usage range may permit the augmented activation of DC fibers. The recruited DC area and the symmetry of DC recruitment increased. With a smaller lead separation, the combined electric fields increased and the DCs were easier to stimulate (evident from the larger depth and width of the recruited DC area) at the expense of medio-lateral steerability. The triple lead configuration -with 1mm transverse separation between the leads - had the largest DC recruited area and usage range for the whole range of CCRs (Fig. 8). Because $1 \mathrm{~mm}$ was the smallest transverse lead separation tested by modeling, a smaller separation might give an even better performance. However, the steering capability and surgical feasibility of such configurations is remote and hence none were simulated. 
The results from Figure 6a and Figure 6b may be obvious but it leads to a non-obvious result in Figure 7 and Figure 8. It is a common notion among physicians that if leads are placed far apart from each other, a larger DC area will be recruited. However this study shows the opposite result. Leads placed closer to each other obtain a larger usage range and recruit a larger DC area. Also, by programming the leads as longitudinal guarded cathodes with a small lateral lead separation, it may compensate for the negative effect of the lateral leads being close to the DRs. When the transversal separation between the leads was large, the CCR had a greater influence on the thresholds. In clinical terms, the sensations of paresthesia and discomfort are more sensitive to changes in current for larger transverse lead separations.

\subsubsection{Anodal steering}

In our modeling study, VAR provided better stimulation outcomes than CAR. The recruitment of DCs was improved and an increased coverage of the DC area was achieved; a larger usage range was maintained throughout the CCR, by suppressing the activation of DRs (Fig. 5). For VAR, the $\mathrm{I}_{\mathrm{DC}}$ was low and $I_{D R}$ was high for all CCRs (Fig. 4). In the VAR configuration, the total current is balanced in the rostro-caudal direction with all CCRs. This means that all the current flows between the electrodes (cathodes and anodes) and is oriented in a rostro-caudal direction, which is not the case if the current is not balanced across the lead (CAR). A predominant, rostro-caudal current flow coincides with the orientation of fibers in the DCs, causing a decrease in their activation thresholds, thus facilitating their stimulation. The superiority of a longitudinal guarded cathode configuration has been confirmed by clinical data (2) and can be explained by the fact that the activating function of the anodes may superimpose on the activating function of the central cathode, thereby increasing the overall activating function near the cathode and reducing the excitation threshold of DC fibers (5).

The $\mathrm{I}_{\mathrm{DR}}$ stayed surprisingly high for VAR. The idea of steering the anodal currents proportional to the cathodal currents in the respective leads was intended to decrease the $\mathrm{I}_{\mathrm{DC}}$; however the DRs appeared to be more sensitive to the direction of current flow. Throughout the steering range from a medial to a lateral direction, the DRs were more difficult to stimulate when the currents were balanced. The difference between thresholds was high when a higher percentage of the current was applied to just one of the cathodes (maximum when CCR is 100\%) but became less when the current was spread between two cathodes (Fig. 4). At CCR of 100\%, non-balancing of currents exposes the lateral leads with $50 \%$ anodal current in CAR and with none of the anodal currents in VAR. It was thought that the shielding effect of the anodal currents on the DRs would reduce lateral recruitment and yield a higher usage range in CAR. However the guarding effect of the longitudinal tripole along with the balancing of currents across the lead in VAR was dominant than the anodal shielding effect in CAR and enabled a higher usage range (due to a higher $\mathrm{I}_{\mathrm{DR}}$ and lower $\mathrm{I}_{\mathrm{DC}}$ ) and DC recruited area. When the current was spread among the 
cathodes, the effect of anodal steering was less pronounced and the performance of the two techniques (CAR and VAR) becomes similar.

\subsubsection{Paddle leads and effect of pulse width}

This modeling study was performed only with percutaneous leads. The percutaneous leads were placed just behind the dura mater, a criterion very easily achieved by modeling, but not guaranteed in clinical practice. A position of these leads dorsally in the epidural fat will result in a strong deterioration of their performance (9). The attained results with percutaneous leads also show that transverse lead separation is vital in affecting paresthesia coverage. The above mentioned reasons can be arguments in favor of paddle lead placement and design, particularly in the low-thoracic vertebral levels. However, the current fields and paresthesia distributions will be different with the paddle leads. Therefore, before any concrete conclusions can be made, paddle leads with similar configurations must be modeled and simulated.

In recent clinical studies by Holsheimer et al. $(29,30)$, the relationship between pulse width of stimulation and paresthesia area was observed. The total paresthesia area increased and extended caudally with increasing pulse width. Holsheimer et al. proposed a theoretical explanation of this phenomenon based on the different medio-lateral distributions of large and small fibers in the DCs. The authors of this article believe that altering pulse width might also have an influence on the medio-lateral extent of DC recruitment using triple lead longitudinal guarded cathodes. However, our presently used spinal cord stimulation model does not include these mechanisms yet and therefore cannot explain these clinical observations.

\subsection{Conclusion}

Triple leads programmed to perform as longitudinal guarded cathodes provide more post-operative flexibility than single and dual leads in covering a larger width of the low-thoracic DCs and a larger lower limb area covered with paresthesia. VAR always enabled increased DC recruitment than CAR, by balancing the currents in the rostro-caudal direction. The transverse separation between the leads is a major determinant in the area and distribution of paresthesia. 


\section{References}

[1] Barolat G. Spinal cord stimulation for chronic pain management. Arch Med Res. 2000;31:258-262.

[2] North RB, Ewend MG, Lawton MT, Piantadosi S. Spinal cord stimulation of chronic, intractable pain: superiority of 'multi-channel' devices. Pain. 1991;44:119-130.

[3] North RB. Spinal cord stimulation for chronic, intractable pain. Adv neurol. 1993; 63:289-301.

[4] Holsheimer J, Struijk JJ, Tas NR. Effects of electrode geometry and combination on nerve fiber selectivity in spinal cord stimulation. Med Biol Eng Comput. 1995;33: 676-682.

[5] Holsheimer J. Effectiveness of spinal cord stimulation in the management of chronic pain: analysis of technical drawbacks and solutions. Neurosurgery. 1997;40:990-999.

[6] Struijk JJ, Holsheimer J, Boom HBK. Excitation of dorsal root fibers in spinal cord stimulation: a theoretical study. IEEE Trans Biomed Eng. 1993;40:632-639.

[7] Holsheimer J, Wesselink WA. Effect of anode-cathode configuration on paresthesia coverage in spinal cord stimulation. Neurosurgery. 1997;41:654-659.

[8] Holsheimer J, Wesselink WA. Optimum electrode geometry for spinal cord stimulation: the narrow bipole and tripole. Med Biol Eng. Comput. 1997;35:493-497.

[9] Manola L, Holsheimer J, Veltink P. Technical performance of percutaneous leads for spinal cord stimulation: a modeling study. Neuromodulation. 2005;8:88-99.

[10] North RB, Kidd DH, Olin J, Sieracki JM, Farrokhi F, Petrucci L et al. Spinal cord stimulation for axial low back pain: a prospective, controlled trial comparing dual with single percutaneous electrodes. Spine. 2005; 30:1412-1418.

[11] Feirabend HKP, Choufoer H, Ploeger S, Holsheimer J, Van Gool JD. Morphometry of human superficial dorsal and dorso-lateral column fibres: significance to spinal cord stimulation. Brain. 2002;125:1137-1149.

[12] Law JD. Spinal stimulation: Statistical superiority of monophasic stimulation of narrowly separated, longitudinal bipoles having rostral cathodes. Appl. Neurophysiol. 1983;46:129-137.

[13] Law JD. Targeting a spinal stimulator to treat failed back surgery syndrome. Appl. Neurophysiol. 1987;50:437-438.

[14] Alo K, Varga C, Krames E, Prager J, Bradley K. Variability of contact impedance by vertebral placement in spinal cord stimulation. Abstracts of the $54^{\text {th }}$ Congress of Neurological Surgeons San Francisco, CA, October 16-21, 2004.

[15] Kreis PG, Fishman SM. Spinal cord stimulation: percutaneous implantation techniques. Oxford University Press 2009.

[16] McNeal DR. Analysis of a model for excitation of myelinated nerve. IEEE Trans Biomed Eng. 1976;23:329-337. 
[17] Frankenhaeuser B, Huxley AF. The action potential in the myelinated nerve fiber of xenopus laevis as computed on the basis of voltage clamp data. $J$ Physiol. 1964;171:302-315.

[18] Struijk J, Holsheimer J, van der Heide G, Boom HBK. Recruitment of dorsal column fibers in spinal cord stimulation: influence of collateral branching. IEEE Trans Biomed Eng. 1992;39:903-912.

[19] Wesselink WA, Holsheimer J, Boom HBK. A model of the electrical behaviour of myelinated sensory nerve fibres based on human data. Med Biol Eng Comput. 1999;37:228-235.

[20] Fyffe REW. Afferent fibers. In:Davidoff R A, editor Handbook of the spinal cord. New York: Marcel Dekker. 1994:79-136.

[21] He J, Barolat G, Ketcik B. Stimulation usage range for chronic pain management. Analgesia 1995; 1:75-80.

[22] Aló KM, Redko V, Charnov J. Four year follow-up of dual electrode spinal cord stimulation for chronic pain. Neuromodulation 2002;5:79-88.

[23] North RB, Fowler K, Nigrin DJ, Szymanski R. Patient interactive computer controlled neurological stimulation system: clinical efficacy in spinal cord stimulator adjustment. J Neurosurg. 1992;76:967972.

[24] North RB, Kidd DH, Olin J, Sieracki JM, Petrucci L. Spinal cord stimulation for axial low back pain: a prospective controlled trial comparing 16-contact insulated electrodes with 4-contact percutaneous electrodes. Neuromodulation. 2006;9:56-67.

[25] Struijk JJ, Holsheimer J. Transverse tripolar spinal cord stimulation: theoretical performance of a dual channel system. Med Biol Eng Comput. 1996;34:273-279.

[26] Holsheimer J, Nuttin B, King GW, Wesselink WA, Gybels JM, de Slutter P. Clinical evaluation of paresthesia steering with a new system for spinal cord stimulation. Neurosurgery. 1998;42:541-549.

[27] Oakley JC, Espinosa F, Bothe H, McKean J, Allen P, Burchiel K et al. Transverse tripolar spinal cord stimulation: results of an International multicenter study. Neuromodulation. 2006;9:192-203.

[28] Wesselink WA, Holsheimer J, King GW, Torgerson NA, Boom HBK. Quantitative aspects of the clinical performance of transverse tripolar spinal cord stimulation. Neuromodulation. 1999;2:5-14.

[29] Holsheimer J, Buitenweg JR, Das J, de Sutter P, Manola L, Nuttin B. The effect of pulse width and contact configuration on paresthesia coverage in spinal cord stimulation. Neurosurgery. 2011;68:1452-1461.

[30] Lee D, Hershey B, Bradley K, Yearwood T. Predicted effects of pulse width programming in spinal cord stimulation: a mathematical modeling study. Med Biol Eng Comput. 2011;49:765-774. 
Chapter 2 


\title{
CHAPTER 3
}

\section{Electrode alignment of transverse tripoles using a percutaneous triple lead approach in SCS}

\author{
Vishwanath Sankarasubramanian, Jan. R. Buitenweg, Jan Holsheimer, Peter Veltink \\ MIRA, Institute for Biomedical Technology and Technical Medicine, University of Twente, Enschede, \\ The Netherlands
}

Published in J Neural Eng., 8(1):016010, January 2011 


\begin{abstract}
Objective: The aim of this modeling study is to determine the influence of electrode alignment of transverse tripoles on the paresthesia coverage of the pain area, in spinal cord stimulation (SCS), using a percutaneous triple- lead approach.

Methods: Transverse tripoles, comprising a central cathode and two lateral anodes, were modeled on the low-thoracic vertebral region (T10-T12) using percutaneous triple-lead configurations, with the center lead on the spinal cord midline. The triple leads were oriented both aligned and staggered. In the staggered configuration, the anodes were offset either caudally (caudally-staggered) or rostrally (rostrallystaggered) with respect to the midline cathode. Transverse tripolar field steering with the aligned and staggered configurations enabled the estimation of dorsal column fiber thresholds $\left(\mathrm{I}_{\mathrm{DC}}\right)$ and dorsal root fiber thresholds $\left(\mathrm{I}_{\mathrm{DR}}\right)$ at various anodal current ratios.
\end{abstract}

Results: $I_{D C}$ and $I_{D R}$ were considerably higher for the aligned transverse tripoles as compared to the staggered transverse tripoles. The aligned transverse tripoles facilitated deeper penetration into the medial dorsal columns (DCs). The staggered transverse tripoles always enabled broad and bilateral DC activation, at the expense of medio-lateral steerability. The largest DC recruited area was obtained with the rostrally-staggered transverse tripole.

Conclusions: Transverse tripolar geometries, using percutaneous leads allow selective targeting of either medial or lateral DC fibers, if and only if the transverse tripole is aligned. Steering of anodal currents between the lateral leads of the staggered transverse tripoles cannot target medially-confined populations of DC fibers in the spinal cord. An aligned transverse tripolar configuration is strongly recommended, because of its ability in providing more post-operative flexibility than other configurations. 


\subsection{Introduction}

Spinal cord stimulation (SCS) is a clinically established neuromodulation technique increasingly used in the treatment of chronic pain. The success of SCS in suppressing chronic pain is determined primarily by the ability in directing the paresthesias to the painful areas (1-3). The conventional methods employed in SCS use longitudinal electrode arrays driven by a single-channel pulse generator (4). The dorsal column (DC) area is targeted by selecting the best anode-cathode combinations and their positions with respect to the spinal cord. With the Transverse Tripolar Lead (TTL) configuration published in 1996 the concept of transverse steering of the electric field combined with anodal shielding of dorsal roots (DRs) on either side $(5,6)$ was introduced. The TTL is a paddle lead with a transverse guarded cathode (central cathode and an anode on either side) driven by a dual-channel pulse generator. Computer modeling studies (5) have demonstrated that the TTL in combination with the dual-channel pulse generator is able to preferentially stimulate segments of spinal cord DCs, and may be effective for the relief of low-back pain in patients with Failed Back Surgery Syndrome. Clinical trials validated the computational model and confirmed that the configuration is indeed successful in shifting the paresthesia to various anatomical locations $(4,7,8)$.

In spite of having a high degree of freedom over the activation of DC fibers and changing the topography of paresthesias, the TTL is limited in its application. This is because commercial systems used only a single source to generate the stimulation field. The full capability of the TTL configuration could not be exploited, resulting in lack of sufficient clinical trials. Moreover, the TTL is implanted in patients through a laminotomy or laminectomy procedure. Laminotomy/laminectomy demands open surgery to allow both access to the dura and proper positioning of the SCS paddle (9). Additionally, laminotomy/laminectomy poses the risk of extensive surgical trauma and other complications (10). Due to the relatively complicated nature of the surgical procedure, the procedure is typically performed only by a neurosurgeon.

Percutaneous leads, on the other hand, can be placed in the dorsal epidural space by a minimally invasive approach. The technique offers relatively easy access to multiple vertebral levels (T9-T12), facilitating paresthesia mapping during positioning (11). The inherent structure of the spine is thereby not altered, less surgery time is required and most importantly the recovery time is shorter. If the transverse tripole is designed to permit implantation using the percutaneous route, both anesthesiologists and neurosurgeons could perform the implant. Hence, a practical solution would be to construct a TTL configuration using three percutaneous leads (figure 1). 


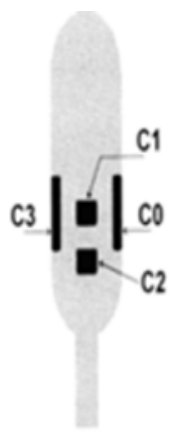

(a)

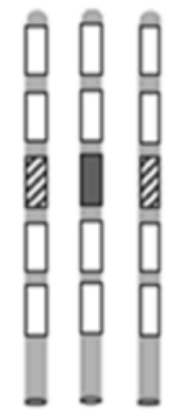

(b)

Figure 1 (a) TTL paddle designed by Struijk and Holsheimer (5). Lateral anodes are longer but thinner than the central cathode to keep the electrode contact surface at $12 \mathrm{~mm}^{2}$. (b) Transverse tripole constructed using three percutaneous leads. Only the contacts marked are used for stimulation. The anodes are hatched and the cathode is filled. The anodes have the same size as the cathode. The extra contact C2 in figure 1(a) enabling stimulation with a central bipole (C1-C2) is not modeled.

Although the insertion technique of percutaneous leads itself is indeed convenient, positioning the leads inside the dorsal epidural space is rather difficult and not always successful (12). When multiple percutaneous leads are inserted, the ends of the leads must be adjusted precisely at the desired location to maintain both the longitudinal orientation and spacing of its contacts. Moreover, percutaneous leads are prone to longitudinal (non-alignment) or lateral migration (asymmetrical lead positions) which reduce or eliminate pain/paresthesia overlap $(11,13,14)$. The percutaneous version of the TTL can cope with lateral lead migrations by transverse steering of the electrical currents. Therefore, the three percutaneous leads mainly need to be sufficiently aligned longitudinally to mimic the configuration of a transverse tripole. If the optimal transverse geometry is not achieved, due to a rostro-caudal offset (staggering/non-alignment) of the lateral leads, will the stimulation result in a decrease or loss of the therapeutic effect? With the development of technology, multichannel current-controlled stimulators are designed and are being introduced into SCS practice. Unlike a single-channel, voltage-controlled stimulator, a multichannel, current-controlled stimulator provides the possibility to apply different currents to each contact independently. Thus, it has become of practical interest to see how much a group of recruited DC fibers can be altered by means of electrical steering with different lead configurations and combinations.

Computer-based models that mimic the behavior of spinal cord structures are useful tools to predict the effect of electrical stimuli on the activation of neural structures (5,15-17).This computer modeling study aims to explore the influence of electrode alignment of transversely oriented guarded cathodes on the paresthesia coverage of the pain area. For this purpose, perfectly aligned and staggered configurations of transverse tripoles, built up from triple percutaneous leads are modeled. 


\subsection{Methods}

The University of Twente Spinal Cord Stimulation software (UT-SCS) is used in this modeling study. This software permits the implementation of a three-dimensional volume conductor model of the spinal column, including electrode arrays and nerve fibers $(18,19)$.

\subsubsection{Volume conductor model}

The volume conductor model consists of a spinal cord model around vertebral levels T10-T12. Its transverse section is shown in figure 2. Thickness of the dorso-medial CSF layer (dCSF) was $3.2 \mathrm{~mm}$. The geometries and electrical conductivities of the human anatomical structures from earlier modeling studies were used (18). The total dimension of the model was $24.2 * 25.7 * 59.35 \mathrm{~mm}$ divided into $64 * 64 * 80$ non-equidistant cubic elements in the medio-lateral, dorso-ventral and rostro-caudal direction respectively. Three different configurations of three percutaneous leads were modeled.

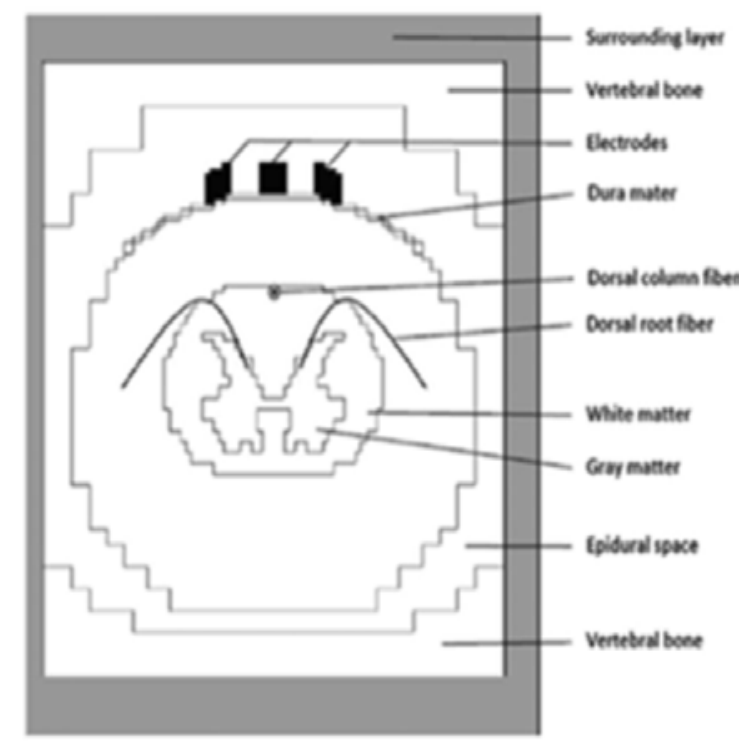

Figure 2 Transverse section of the UT-SCS volume conductor model of the low-thoracic spinal cord with epidural electrodes and DRs.

\subsubsection{Percutaneous SCS lead types}

For all three configurations, percutaneous leads (model Advanced Bionics AB SC2108) were used. Although there are 5 contacts per lead, only one of the contacts was used for stimulation. The anodes are hatched and the cathodes are filled (figure 1(b) and figure 3(a-c)).The cylindrical contacts with a diameter of $1.35 \mathrm{~mm}$ were modeled as four rectangular surfaces around an insulating square bar. The length of the contacts was chosen to be $3 \mathrm{~mm}$ to as in the clinical lead. The two transverse dimensions of the contacts were chosen to be $1.0 \mathrm{~mm}$ each. The insulation inside the contacts was modeled as a low-conductive $(0.0001 \mathrm{~S} / \mathrm{m})$ compartment. The leads were placed inside the dorsal epidural space adjacent to the dura 
mater. As mentioned earlier, a triple lead configuration was used in all models. On the center lead, an electrode is defined as the cathode. On both lateral leads, a shielding anode is defined. The anodal contacts have the same length as the cathode. The electrode configuration is placed at the midline of the spinal column.

Aligned transverse tripoles. This triple lead configuration is modeled with the cathode and anodes at the same rostro-caudal level (aligned). The gap or medio-lateral spacing between the adjacent leads is $1.5 \mathrm{~mm}$ edge-to-edge. The resulting medio-lateral span, defined as the center-to-center distance of the outer electrodes, is $5.0 \mathrm{~mm}$. Hence, the configuration represents a transverse guarded cathode. A schematic overview of this model is shown in figure 3a.

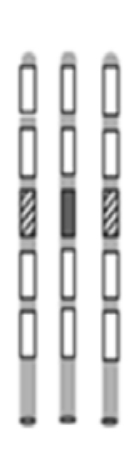

(a)

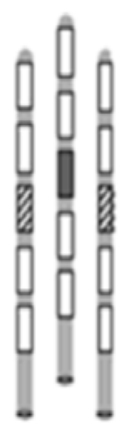

(b)

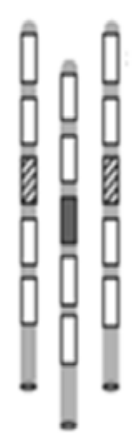

(c)

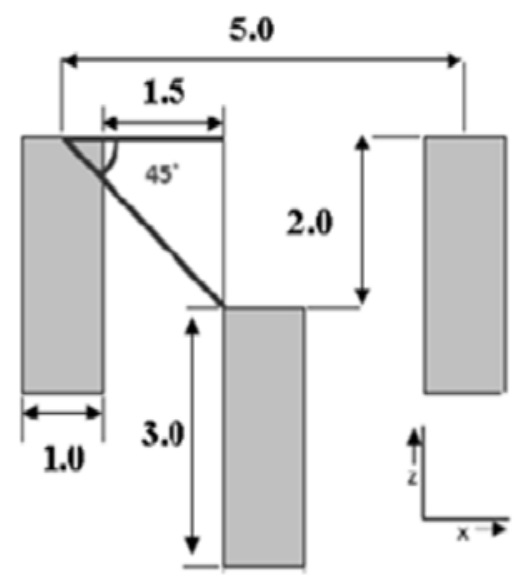

(d)

Figure 3 (a) Aligned transverse guarded cathode. The cathodes and anodes are at the same rostrocaudal level. (b) Caudally-staggered transverse guarded cathode. The anodes are shifted $2.0 \mathrm{~mm}$ caudally with respect to the central cathode. (c) Rostrally-staggered transverse guarded cathode. The anodes are shifted $2.0 \mathrm{~mm}$ rostrally with respect to the central cathode. (d) Schematic overview of the rostrallystaggered transverse guarded cathode. Dimensions are in millimeters. The anodes are hatched.

Staggered transverse tripoles. Two staggered transverse tripolar configurations are defined, both having the center lead with the cathode positioned on the spinal cord midline.

In the first configuration the anodes are offset caudally with respect to the midline cathode by $2 \mathrm{~mm}$, as shown in figure 3(b). In the second configuration, the anodes are offset rostrally by $2 \mathrm{~mm}$ with respect to the cathode as shown in figure 3(c).

In earlier modeling studies (5), the field in the DCs had only been calculated for anodal ratios 50/50\% (symmetrical) and 100/0, 0/100\% (extreme asymmetry). It is not known how smooth the shape of the 
electric field varies when small steps in anodal ratios are given. Hence, in this study, across all 3 configurations, transverse electric field steering was modeled by varying the ratio of anodal currents in small increments of 10\% each (0/100, 10/90, 20/80, 30/70, 40/60, 50/50\% and vice-versa till 100/0\%). These numbers represent the percentage of anodal current applied to the right lead anode and the left lead anode respectively. The pulse width was 210 usec.

Because of the variability in clinically positioning the three percutaneous leads, an additional set of simulation (0/100, 20/80, 50/50, 80/20 and 100/0\%) was performed for the caudally and rostrally staggered configurations, offset $1 \mathrm{~mm}$ with respect to the midline cathode.

\subsubsection{Nerve fiber model}

DC and DR nerve fibers were positioned in the volume conductor model. The DC and DR fibers used in this study have the following standard diameters:

DC fibers (without collaterals): $12 \mu \mathrm{m}$ in the median $66 \%$ of the DCs and linearly increasing to $15 \mu \mathrm{m}$ at the lateral borders, thus mimicking the lower fiber threshold due to the presence of an increasing density of collaterals near the bifurcation of the corresponding DR fibers $(20,21)$. The longitudinal DC-fiber is placed just below the dorsal border of the DCs. The DC fiber diameter is assumed to be the same independent of fiber depth.

DR fibers: $15 \mu \mathrm{m}$, with an ascending and a descending $12 \mu \mathrm{m}$ DC fiber with collaterals attached. Since a low-thoracic segment was modeled, we chose to model the 'type A1' DR fiber, as described by Struijk et

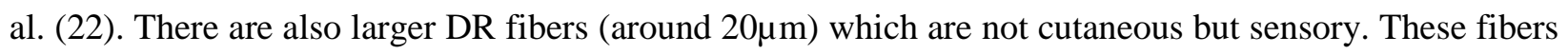
have other functions, such as in reflex movements, and are not involved in the perception of paresthesia. Therefore, these fibers were not modeled and simulated.

The curvature of the left and right DR fiber in a transverse plane is shown in figure 4(a). The projections of left and right DR fiber on coronal and sagittal planes are shown in figure 4(b) and figure 4(c) respectively. The parameters of these modeled nerve fibers were taken from earlier studies (16). Transverse electrical field steering with the aligned and staggered configurations enabled the estimation of DC and DR fiber thresholds at various anodal current ratios. Fiber thresholds were calculated at the minimal cathodal current required to elicit an action potential. 
Transverse

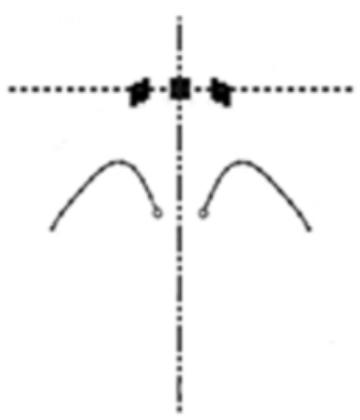

(a)
Coronal

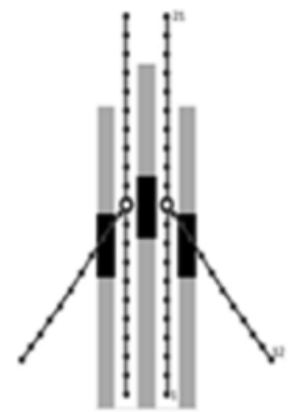

(b)
Sagittal

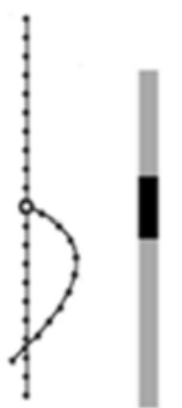

(c)

Figure 4 Cross sectional view of the volume conductor model showing the spatial relation between electrodes and DR fibers. Projections of the left and the right curved DR fiber in a (a) transverse plane (b) coronal plane and (c) sagittal plane. The longitudinal fiber consists of 21 nodes. The curved fiber consists of 11 nodes and has a diameter of $15 \mu \mathrm{m}$. The curved fiber is attached to the $11^{\text {th }}$ node of the longitudinal fiber. The collaterals are not shown.

\subsubsection{Outcome variables}

The implementation of the fiber model and its connection with the solution of the volume conductor model enables the analysis of several clinically relevant SCS outcome variables.

- $\mathrm{I}_{\mathrm{DC}}(\mathrm{mA})$ : DC fiber activation threshold, corresponding to the lowest activation threshold among all DC fibers

- $\mathrm{I}_{\mathrm{DR}}(\mathrm{mA})$ : DR fiber activation threshold, corresponding to the minimum of $\mathrm{I}_{\mathrm{DR}, \mathrm{L}}$ and $\mathrm{I}_{\mathrm{DR}, \mathrm{R}}$

- $\mathrm{I}_{\mathrm{PT}}(\mathrm{mA})$ : Perception threshold, corresponding to the current required to activate the lowest threshold fiber, being either a DC or DR fiber. Hence, it is calculated as the lowest value between $\mathrm{I}_{\mathrm{DC}}$ and $\mathrm{I}_{\mathrm{DR}}$

- $\mathrm{I}_{\mathrm{DT}}$ (mA): Discomfort threshold, corresponding to the current at initial stimulation of proprioceptive DR fibers. It is defined as $\mathrm{I}_{\mathrm{DT}}=1.4 * \mathrm{I}_{\mathrm{DR}}(23)$.

- UR: Usage range, defined as the ratio between $\mathrm{I}_{\mathrm{DT}}$ and $\mathrm{I}_{\mathrm{PT}}$ for which initial paresthesia is perceived. UR $=\mathrm{I}_{\mathrm{DT}} / \mathrm{I}_{\mathrm{PT}}$. The UR is an indicator of the extent of DC activation.

- $\mathrm{S}_{\mathrm{RA}}\left(\mathrm{mm}^{2}\right)$ : Maximum recruited DC area, which is the region in the spinal cord comprising the DC fibers that are activated at $\mathrm{I}_{\mathrm{DT}}$.

- W (mm): Width of recruited area, defined as the maximum medio-lateral extent of the recruited $\mathrm{DC}$ area at $\mathrm{I}_{\mathrm{DT}}$

- D (mm): Depth of recruited area, defined as the maximum dorso-ventral extent of the recruited $\mathrm{DC}$ area at $\mathrm{I}_{\mathrm{DT}}$. 
- $\mathrm{AS}_{\mathrm{RA}}(\%)$ : Asymmetry of the recruited DC area, defined as the percentage of $\mathrm{S}_{\mathrm{RA}}$ in either the left or right DC.

$I_{D C}$ and $I_{D R}$ are the first outcome variables to be computed. The threshold of the DC-fiber is evaluated at several medio-lateral positions, since the x-coordinate of the fiber having the lowest threshold is expected to vary with the anodal current ratio. $\mathrm{I}_{\mathrm{DC}}$ is taken to be the lowest value from all the computed DC fiber thresholds.

$I_{D R}$ is calculated as the minimum of $I_{D R, L}$ and $I_{D R}, R$. In order to obtain these thresholds, it is necessary that the respective fiber systems are displaced (here in steps of $1 \mathrm{~mm}$ ) in a rostral and caudal direction, until the lowest threshold is found. The procedure is justified by the fact that, at one spinal level, there is only one DR. The DRs, then split up into different rootlets, which enter the dorsal root entry zone (DREZ) at different angles and rostro-caudal intervals of approximately $1 \mathrm{~mm}(18,24)$. Therefore, moving the fiber systems rostrally and caudally results in finding the rootlet having the lowest threshold.

\subsection{Results}

\subsubsection{Effect of transverse tripole orientation on nerve fiber thresholds and usage range}

The effects of varying the anodal currents on the $\mathrm{I}_{\mathrm{DC}}$ and $\mathrm{I}_{\mathrm{DR}}$, as well as usage range were analyzed for both aligned and staggered triple lead configurations. Because of the variability in clinically positioning the three leads, some indication of the range in output variables ( $\mathrm{I}_{\mathrm{DC}}, \mathrm{I}_{\mathrm{DR}}$, and usage range) due to changes in the rostro-caudal distances (for both $1 \mathrm{~mm}$ and $2 \mathrm{~mm}$ ) between the leads was thought to be useful.

$\mathrm{I}_{\mathrm{DC}}$ for the aligned and staggered configurations as a function of the current ratio in the left anodes is showed in figure $5 . \mathrm{I}_{\mathrm{DC}}$ had a minimum value when the entire current was applied to one of the anodes (0/100\%), and reached a maximum value when the stimulation was symmetrical (50/50\%). The values of $\mathrm{I}_{\mathrm{DC}}$ were considerably higher for the aligned as compared to the staggered tripoles (the maximum difference of 56.8\% was observed between the aligned and the $2 \mathrm{~mm}$ offset staggered transverse tripole at the $50 / 50 \%$ current ratio). There is no difference in the $I_{D C}$ values of the rostrally-staggered and caudallystaggered transverse tripoles and hence their DC activation threshold curves overlap (for both 1mm and 2mm offsets). 


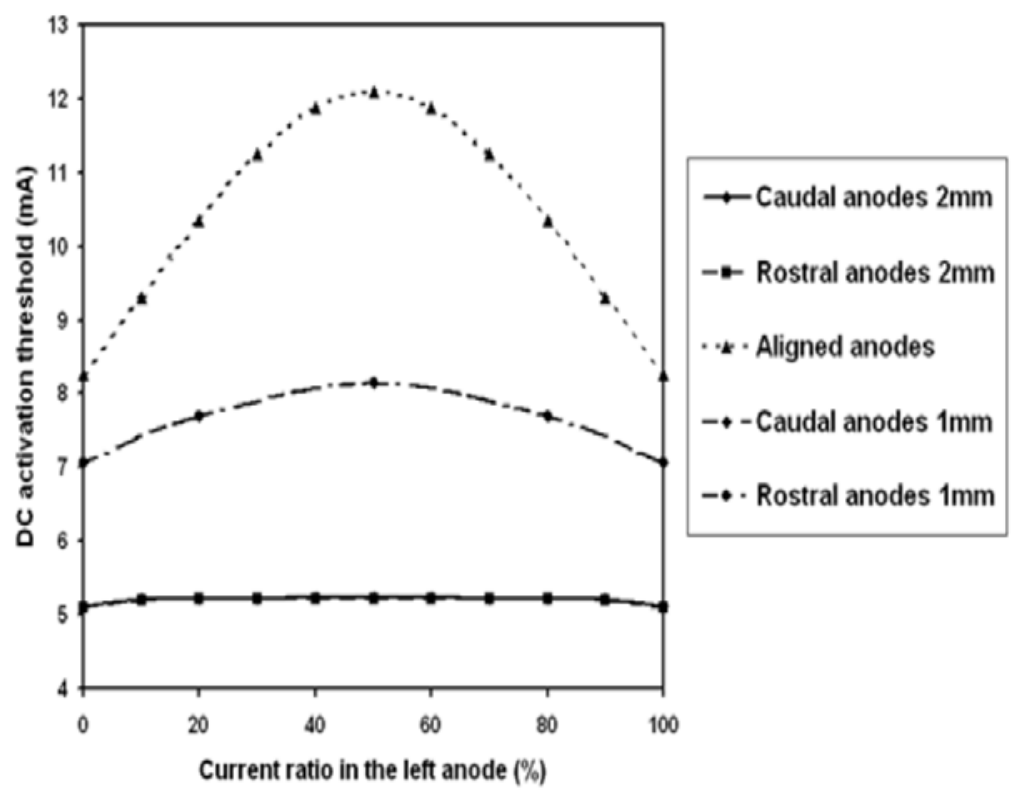

Figure 5 Variation of $I_{D C}(m A)$ with the percentage of current applied to the left anode (\%), for all the five transverse guarded cathode configurations (rostrally and caudally-staggered, both $1 \mathrm{~mm}$ and $2 \mathrm{~mm}$, and aligned). The curves of the rostrally and caudally-staggered anodes (for both $1 \mathrm{~mm}$ and $2 \mathrm{~mm}$ offsets) overlap and hence is seen as one curve. As a result, there are three curves instead of five.

$I_{D R}$ for the five configurations as a function of their anodal current ratio is shown in figure 6(a). $I_{D R}$ had a minimum when the entire current was applied to one of the anodes. $\mathrm{I}_{\mathrm{DR}}$ reached a maximum, when the stimulation was symmetrical (50/50\% ratio). $\mathrm{I}_{\mathrm{DR}}$ was largest for the aligned tripoles at symmetrical stimulation (equal anodal currents on either side). Moreover, the differences in $I_{D R}$ values for the aligned tripoles were substantial at particular anodal current ratios (38\% increase from 20/80 to 30/70 and 51.1\% increase from $30 / 70$ to $40 / 60 \%$ and vice versa) showing an increased sensitivity of the DR fiber to variations in anodal current densities (figure 6(a)). $\mathrm{I}_{\mathrm{DR}}$ and its sensitivity to certain current ratios were low for the staggered orientation of the tripoles (figure 6(a)). Also, inverting the orientation of the tripoles yielded a different $I_{D R}$. The rostrally-staggered configuration exhibited higher $I_{D R}$ than the caudallystaggered configuration for the entire range of current ratios (the maximum difference of $22.7 \%$ for the $2 \mathrm{~mm}$ offset configuration at the 50/50\% current ratio), as shown in figure $6(\mathrm{a})$. The $\mathrm{I}_{\mathrm{DR}}$ of the $1 \mathrm{~mm}$ offset staggered configurations were lower than that of the aligned configuration and higher as compared to the $2 \mathrm{~mm}$ offset staggered configurations.

At anodal current ratios 0/100 and 100/0\%, $\mathrm{I}_{\mathrm{DR}}$ was less than $\mathrm{I}_{\mathrm{DC}}$, indicating preferential DR recruitment. At all other anodal current ratios of both aligned and staggered configurations, $\mathrm{I}_{\mathrm{DC}}$ was lower than $\mathrm{I}_{\mathrm{DR}}$, 
and hence the perception threshold $\left(\mathrm{I}_{\mathrm{PT}}\right)$ was determined by $\mathrm{I}_{\mathrm{DC}}$. The absolute values of usage range and their sensitivities to anodal current ratios are shown in figure 6(b) respectively. The overall profiles of $I_{D R}$ and usage range for all five configurations were similar. UR reached a maximum, when the stimulation was symmetrical (50/50\% ratio). UR was largest for the aligned tripoles at symmetrical stimulation (equal anodal currents on either side).

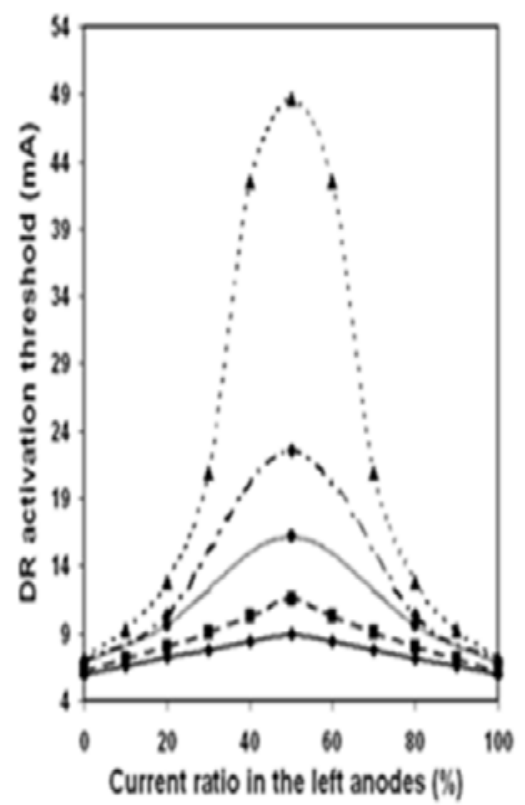

(a)

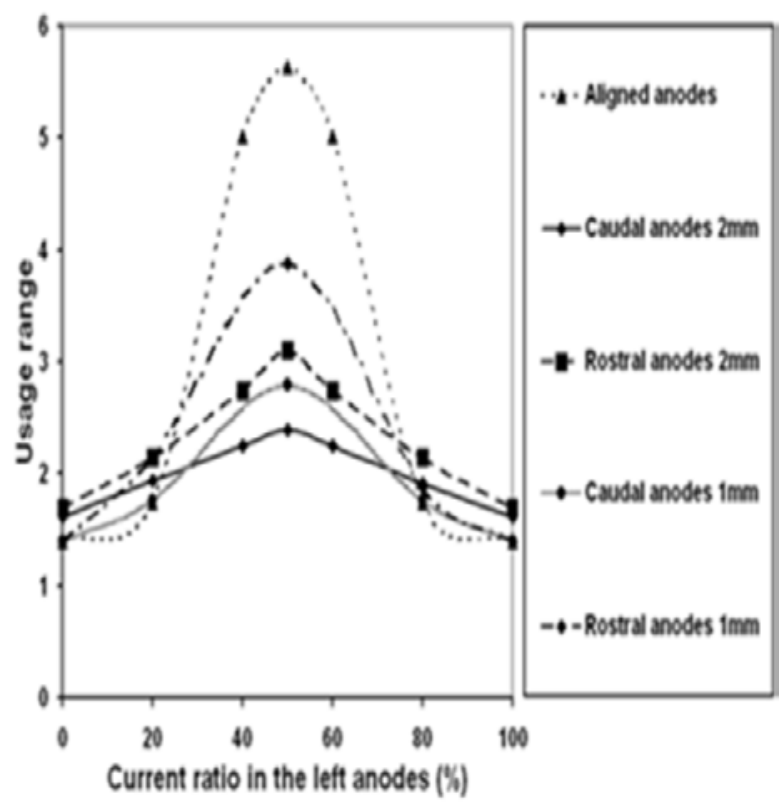

(b)

Figure 6 (a) $I_{D R}$ of all the five transverse guarded cathode configurations as a function of the percentage of current applied to the left anode. (b) UR of the five configurations as a function of the percentage of current applied to the left anode.

\subsubsection{Effect of transverse tripole orientation on dorsal column recruitment}

Figure 7a-d depicts the DC areas recruited at $\mathrm{I}_{\mathrm{DT}}$ for the aligned and staggered configurations, at anodal current ratios of 0/100, 20/80, 40/60 (asymmetrical stimulation) and 50/50\% (symmetrical stimulation) respectively. The asymmetry of DC recruitment is indicated in table 1 for the same anodal current ratios. The depth and width of DC recruitment are plotted in figure 8. 


\section{(a)}

(b)

(c)

(d)

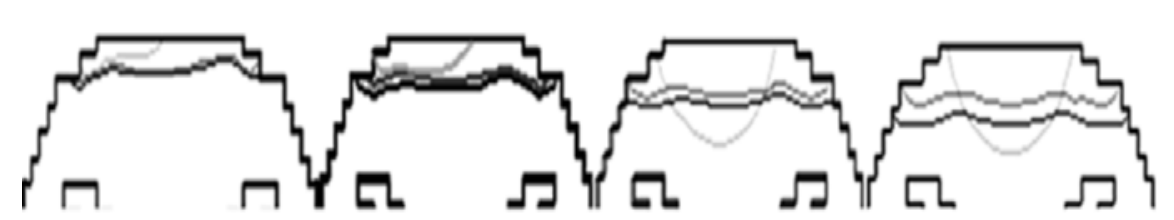

Figure 7 Recruitment contours indicating the ventral boundary of the DC area at $I_{D T}$ for the aligned (light grey line) and staggered (dark grey line for caudally-staggered and black line for rostrallystaggered) configurations, at anodal current ratios of (a) 0/100, (b) 20/80, (c) 40/60 and (d) 50/50\% respectively.

Table 1 Symmetrical (50/50\%) and asymmetrical (0/100, 20/80, 40/60, 60/40, 80/20 and 100/0\%) stimulation with aligned and staggered transverse guarded cathodes. The asymmetry of DC recruitment $\left(A S_{R A}\right)$ for all the three transverse guarded cathode configurations as a function of the percentage of current applied to the left anode. The ratios of 60/40 to 100/0\% are not shown, because they are the same as from $0 / 100$ to $40 / 60 \%$.

\begin{tabular}{ccc}
\hline Parameter & $\begin{array}{c}\text { Anodal current ratio } \\
(\%)\end{array}$ & $\mathbf{A S}_{\mathbf{R A}} \mathbf{( \% )}$ \\
\hline Aligned transverse tripole & $0 / 100$ & $\mathrm{R}=100 \quad \mathrm{~L}=0$ \\
& $20 / 80$ & $\mathrm{R}=91.2 \quad \mathrm{~L}=8.8$ \\
& $40 / 60$ & $\mathrm{R}=64.7 \quad \mathrm{~L}=35.3$ \\
& $\mathbf{5 0 / 5 0}$ & $\boldsymbol{R}=\mathbf{L}=5 \mathbf{0}$ \\
Caudally-staggered & $0 / 100$ & $\mathrm{R}=57 \quad \mathrm{~L}=43$ \\
transverse tripole & $20 / 80$ & $\mathrm{R}=50 \quad \mathrm{~L}=50$ \\
& $40 / 60$ & $\mathrm{R}=49.4 \mathrm{~L}=50.6$ \\
& $\mathbf{5 0 / 5 0}$ & $\mathrm{R}=\mathbf{4 8 . 6} \mathbf{L}=\mathbf{5 1 . 4}$ \\
Rostrally-staggered & $0 / 100$ & $\mathrm{R}=56 \quad \mathrm{~L}=44$ \\
transverse tripole & $20 / 80$ & $\mathrm{R}=51 \quad \mathrm{~L}=49$ \\
& $40 / 60$ & $\mathrm{R}=49.7 \mathrm{~L}=50.3$ \\
& $\mathbf{5 0 / 5 0}$ & $\boldsymbol{R}=\mathbf{4 8 . 9} \mathbf{L}=5 \mathbf{5 1 . 1}$ \\
\hline
\end{tabular}




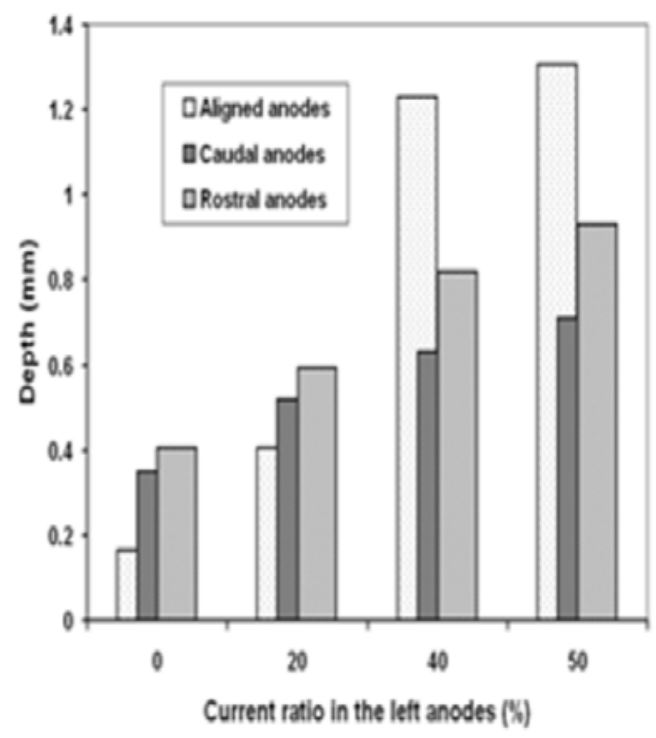

(a)

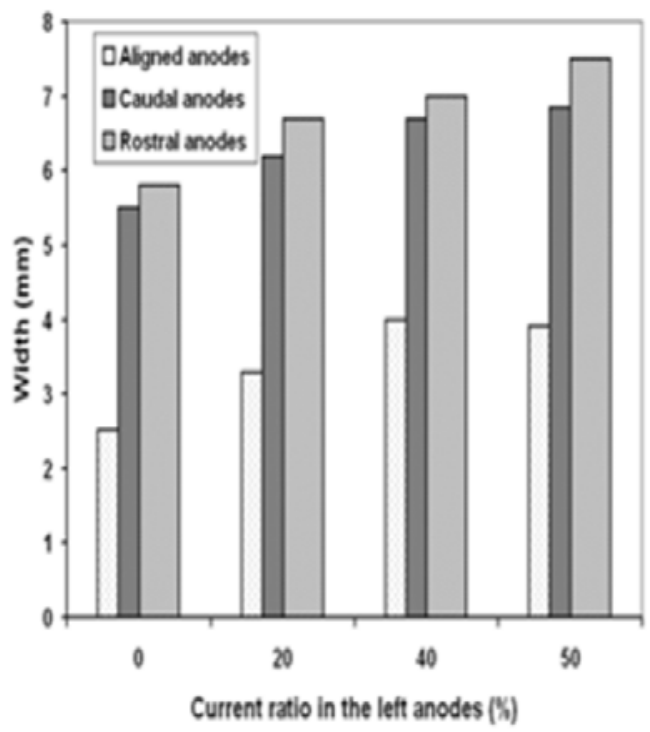

(b)

Figure 8 (a) Depth of all the three transverse guarded cathode configurations as a function of the percentage of current applied to the left anode. (b) Width of the three configurations as a function of the percentage of current applied to the left anode. The ratios of 60/40 to 100/0\% are not shown, because they are the same as from 0/100 to 40/60\%.

For aligned transverse tripoles, the area of activation was restricted to the medial part of the DCs (figure 7d), when equal anodal currents were applied through the lateral leads on either sides of the spinal column midline (symmetrical stimulation). When the anodal current is steered from left lead (0/100\%) to midline lead (50/50\%), the DC recruitment contour moved from right to midline (table $1, \mathrm{AS}_{\mathrm{RA}}$ of right DC decreases from 100 to 50\%); that is, the shift was towards the side having a decreased injection of anodal current, as evident in figure 7a-d and table 1.

The staggered transverse tripoles enabled broad and bilateral DC activation at all anodal current ratios (table 1, the $\mathrm{AS}_{\mathrm{RA}}$ of the left and the right DCs do not differ a lot). This also means that steering of anodal currents between the lateral leads of the staggered transverse tripoles cannot target medially-confined populations of DCs in the spinal cord. The rostrally-staggered transverse tripole, in particular, recruited a larger depth and width of DC area, throughout the current range, as compared to the caudally-staggered transverse tripole configuration (figure 7a-d and figure 8a-b). 


\subsection{Discussion}

The primary aim of this modeling study was to determine the influence of the electrode alignment of transversely oriented guarded cathodes (aligned and staggered guarded cathodes) on the paresthesia coverage of pain area.

\subsubsection{Aligned versus staggered transverse guarded cathode: DC fiber recruitment}

The computer modeling results show that aligned transverse tripoles on percutaneous leads require higher currents for DC activation (figure 5). The reasons for the higher currents are due to the confined electric field evoked by the small cathode-anode distance, and the transverse nature of the current with respect to the orientation of DC fibers. Paresthesia coverage of pain area depends on the part of the DCs where its largest fibers are activated by stimulation. The DC topography is related with the dermatomal topography and pain topography (25). The aligned transverse tripole achieves deeper penetration of the medial DCs with symmetrical stimulation (figure $7 \mathrm{~d}$ and figure $8 \mathrm{a}$ ). Since the $\mathrm{DC}$ recruitment is focused near the midline cathode, paresthesia areas will probably be limited. However, the depth of DC activation is likely to evoke intense paresthesias. When stimulation is applied asymmetrically (unequal anodal currents on either side) across the spinal cord, the symmetry of DC recruitment is largely affected (table 1 , the $\mathrm{AS}_{\mathrm{RA}}$ of right and left DCs differ a lot). The DC recruitment contours shift from right to left (figure 7a-d) as the anodal currents are steered from left to right and also vice-versa. Clinically this means that as steering progresses from one side of the DC s to other, paresthesias shift gradually in a limited number of dermatomes to the other side of the body. The laterality of the stimulation is easily controlled, by current steering using the percutaneous aligned transverse tripolar configuration. Therefore, lead asymmetries can be compensated, which cannot be obtained with a conventional single-channel pulse generator.

The aligned transverse guarded cathode on a percuatenous lead resembles the TTL paddle, both in geometry and in performance. It can be seen from figure 1 and figure 3 that the electrode contact surface of both the configurations is $12 \mathrm{~mm}^{2}$, although the lateral anodes of the TTL are longer and thinner. Performance wise, for the two configurations, it is the increase of usage range, depth of activation of medial DC fibers and symmetry of DC recruitment which are similar. A multiple independent current control (MICC) design would however be preferred over a dual-channel pulse generator because its performance is not affected by unpredictable and time-varying impedances seen by the contacts. A recent clinical case report by Buvanendran et al showed that transverse tripolar stimulation is achieved using 3 percutaneous leads (1 octopolar and 2 quadripolar leads). It contributed to maximum DC stimulation and minimal DR stimulation and provided analgesia to the lower back (26).

The staggered transverse tripoles require lower currents for DC activation as compared to aligned transverse tripoles (figure 5). The $\mathrm{I}_{\mathrm{DC}}$ of the rostrally and caudally staggered configurations (for both $1 \mathrm{~mm}$ and $2 \mathrm{~mm}$ offsets) showed no difference with varying anodal current ratios. The computational 
model also showed that the asymmetry coefficients $\left(\mathrm{AS}_{\mathrm{RA}}\right)$ of DC fibers are only marginally affected (table 1). This implies that steering of anodal currents between the lateral leads of the staggered transverse tripoles cannot exclusively target medially-confined populations of DC fibers in the spinal cord. At the expense of medio-lateral steerability, staggered transverse tripoles always enable broad and bilateral DC activation at a relatively small dorso-ventral depth (figure 7a-d). A wide DC area (figure 8b) in contrast to the aligned transverse tripoles is recruited, which can additionally favor paresthesias in rostral dermatomes. The rostrally-staggered transverse tripole produces the largest recruited DC area at symmetrical stimulation covering a large lateral extent of the DCs at $\mathrm{I}_{\mathrm{DT}}$. The rostro-caudal asymmetry of the curved DR fiber accounts for this unequal threshold and paresthesia distribution (18). Wider DC recruitment can enable multiple dermatomes to be activated beneficially in the reduction of widespread or complex pain.

\subsubsection{Aligned versus staggered transverse guarded cathode: DR fiber recruitment}

The computer modeling results show that aligned transverse tripoles on percutaneous leads, require higher currents for DR fiber activation (figure 6(a)). The higher DR activation thresholds ( $\left.\mathrm{I}_{\mathrm{DR}}\right)$ and discomfort thresholds $\left(\mathrm{I}_{\mathrm{DT}}\right)$ are caused by the shielding of the DRs with the lateral anodes. Clinical evidence to date supports the observation that discomfort sensations are related to activation of fibers in the DRs $(23,27)$. If the three leads are perfectly aligned, the thresholds of radicular responses can be increased significantly. This can be desirable particularly when stimulating at mid-thoracic levels, where DR fiber activation is often a limiting factor (28). The aligned tripoles show a large usage range (figure 6(b)) throughout the medio-lateral span of current ratios and particularly at symmetrical stimulation. The usage range, defined as the ratio of $\mathrm{I}_{\mathrm{DT}}$ and $\mathrm{I}_{\mathrm{PT}}$, is an indicator of the extent of DC activation and thus the extent of maximum paresthesia coverage. An increased number of activation of DC fibers (both medially and laterally) is a result of a larger usage range. The shielding of DRs by the lateral anodes of the aligned transverse tripole considerably increases the $\mathrm{I}_{\mathrm{DR}}$ and $\mathrm{I}_{\mathrm{DT}}$. The high $\mathrm{I}_{\mathrm{DR}}$ increases the usage range thereby allowing penetration into the medial DCs. Lateral anodes limit recruitment of lateral DCs. Since more medial DCs are recruited, paresthesia areas are likely to be limited.

Staggering of lateral leads in the rostro-caudal direction displaces the anodal contacts relative to the cathodes, and hence the electric field. The result is a differential activation of DRs (figure 6(a)). It is known that the DRs, in general, have higher excitability than DCs (29). But the difference depends on several factors, such as electrode configuration and dorsal thickness of CSF (dCSF). When stimulating at the low-thoracic portions of the spine, where the dCSF is generally small, a mixed activation of DC and DR fibers is likely to occur (27). DR stimulation can be identified by segmentary paresthesias and muscle contractions whereas DC stimulation largely evokes paresthesia in multiple dermatomes. The DRs are less shielded by the anodes of the staggered tripoles and require lower currents for their activation (figure 
6(a)). As a result, a wide area of DC recruitment is enabled (figure 7a-d and figure 8b), similar to that obtained with monopolar and longitudinal guarded cathode configurations. Recruitment of lateral fibers in the DCs can additionally extend the paresthesias to rostral body parts. Thus, in spite of reduced DR activation thresholds, a large usage range is obtained (figure 6(b)), due to an increased stimulation of DC fibers.

Quadripolar anodal configurations may be able to confine currents better than dual anodes. For example, a virtual transverse tripole (combination of caudally and rostrally-staggered transverse tripole) with 4 anodes can be effective in reducing the energy consumption and also in increasing the anodal shielding of DRs (30).

The normally high thresholds (both $\mathrm{I}_{\mathrm{DC}}$ and $\mathrm{I}_{\mathrm{DR}}$ ) of aligned and staggered percutaneous triple lead configurations from this computational study as compared to the study by Wesselink et al (8) are probably due to the large dCSF values (3.2mm) assumed at low-thoracic spinal levels. The relatively smaller width or span (center to center distance of the outer electrodes, 5mm) of the percutaneous triple lead configurations could have also accounted for a larger $\mathrm{I}_{\mathrm{DR}}$ (figure 6(a)) as compared to the TTL (8), which has a paddle width of $10 \mathrm{~mm}$.

\subsubsection{Percutaneous versus paddle leads}

Transverse tripolar geometries using percutaneous leads allow selective targeting of either medial or lateral DC fibers, if and only if the transverse tripole is aligned. Steering of anodal currents between the lateral leads of the staggered transverse tripoles cannot exclusively target medio-lateral populations of DC fibers in the spinal cord. A broad and bilateral DC activated area is recruited, similar to that obtained with monopoles and longitudinal guarded cathodes. A three lead configuration (aligned/staggered), like in this case may be appropriate based on the symmetrical characteristics of the pain pattern. An aligned transverse tripolar configuration using percutaneous leads is recommended, because of its ability in providing more post-operative flexibility. Percutaneous leads enable trial stimulation in awake patients to assess the suitability of a permanent implant. This characteristic of percutaneous leads proves advantageous over paddle leads, which are placed under general anesthesia eliminating the patient's feedback on stimulation coverage.

\section{Acknowledgements}

The authors gratefully thank Boston Scientific Neuromodulation (Valencia, CA, USA) for their grant to support this research. 


\section{References}

[1] Barolat G. Spinal cord stimulation for chronic pain management. Arch. Med. Res. 2000;31: 258-262.

[2] North RB, Ewend MG, Lawton MT, Piantadosi S. Spinal cord stimulation of chronic, intractable pain: superiority of 'multi-channel' devices. Pain. 1991;44:119-130.

[3] North RB. Spinal cord stimulation for chronic, intractable pain. Adv. neurol. 1993;63:289-301.

[4] Oakley JC, Espinosa F, Bothe H, McKean J, Allen P, Burchiel K, Quartey G, Spincemaille G, Nuttin B, Gielen F, King G, Holsheimer J. Transverse tripolar spinal cord stimulation: results of an International multicenter study. Neuromodulation. 2006;9:192-203.

[5] Struijk JJ, Holsheimer J. Transverse tripolar spinal cord stimulation: theoretical performance of a dual channel system. Med. Biol. Eng. Comput. 1996;34:273-279.

[6] Struijk JJ, Holsheimer J, Spincemaille GH, Gielen FL, Hoekema R. Theoretical performance and clinical evaluation of transverse tripolar spinal cord stimulation. IEEE Trans. Rehab. Eng. 1998;6:277-285.

[7] Holsheimer J, Nuttin B, King GW, Wesselink WA, Gybels JM, de Slutter P. Clinical evaluation of paresthesia steering with a new system for spinal cord stimulation Neurosurgery. 1998;42:541-549.

[8] Wesselink WA, Holsheimer J, King GW, Torgerson NA, Boom HBK. Quantitative aspects of the clinical performance of transverse tripolar spinal cord stimulation. Neuromodulation. 1999;2:5-14.

[9] Ebel H, Balogh A, Volz M, Klug N. Augmentative treatment of chronic deafferentation pain syndromes after peripheral nerve lesions. Minim. Inv. Neurosurgery. 2000;43:44-50.

[10] North RB, Kidd DH, Petrucci L, Dorsi MJ. Spinal cord stimulation electrode design: a prospective, randomized, controlled trial comparing percutaneous with laminectomy electrodes: part II-clinical outcomes. Neurosurgery. 2005;57:990-996.

[11] North RB, Lanning A, Hessels R, Cutchis PN. Spinal cord stimulation with percutaneous and plate electrodes: side effects and quantitative comparisons. Neurosurg. Focus. 1997;2.

[12] Manola L, Holsheimer J. Technical performance of percutaneous and laminectomy leads analyzed by modeling. Neuromodulation. 2004;7:231-241.

[13] North RB, Kidd DH, Olin JC, Sieracki JM. Spinal cord stimulation electrode design: prospective, randomized, controlled trial comparing percutaneous and laminectomy electrodes-part I: technical outcomes. Neurosurgery. 2002;51:381-390.

[14] Villavicencio A, Leveque J, Rubin L, Bulsara K, Gorecki J. Laminectomy versus percutaneous electrode placement for spinal cord stimulation. Neurosurgery. 2000;46:399-406.

[15] Holsheimer J, Wesselink WA. Optimum electrode geometry for spinal cord stimulation: the narrow bipole and tripole. Med. Biol. Eng. Comput. 1997;35:493-497. 
[16] Wesselink WA, Holsheimer J, Boom HBK. A model of the electrical behaviour of myelinated sensory nerve fibres based on human data Med. Biol. Eng. Comput. 1998;37:228-235.

[17] Manola L, Holsheimer J, Veltink P, Bradley K, Peterson D. Theoretical investigation into longitudinal cathodal field steering in spinal cord stimulation. Neuromodulation. 2007;10: 120-132.

[18] Struijk JJ, Holsheimer J, Boom HBK. Excitation of dorsal root fibers in spinal cord stimulation: a theoretical study. IEEE Trans. Biomed. Eng. 1993;40:632-639.

[19] Holsheimer J. Computer modeling of spinal cord stimulation and its contribution to therapeutic efficacy (Review). Spinal cord. 1998;36:531-540.

[20] Struijk J, Holsheimer J, van der Heide G, Boom HBK. Recruitment of dorsal column fibers in spinal cord stimulation: influence of collateral branching. IEEE Trans. Biomed Eng. 1992;39:903-912.

[21] Fyffe REW. Afferent fibers. In: Davidoff RA, editor Handbook of the spinal cord. New York: Marcel Dekker. 1994;79-136.

[22] Struijk JJ, Holsheimer J and Boom HBK. Excitation of dorsal root fibers in spinal cord stimulation: a theoretical study. IEEE Trans. Biomed. Eng. 1993;40:632-639.

[23] He J, Barolat G and Ketcik B. Stimulation usage range for chronic pain management. Analgesia. 1995;1:75-80.

[24] Carpenter MB. Core text of neuroanatomy. Baltimore: Williams \& Wilkins. 1972.

[25] Barolat G, Massaro F, He J, Zeme S, Ketcik B. Mapping of sensory responses to epidural stimulation of the intraspinal neural structures in man. J Neurosurg. 1993;78:233-239.

[26] Buvanendran A, Lubenow TJ. Efficacy of transverse tripolar spinal cord stimulator for the relief of chronic low back pain from failed back surgery. Pain Physician. 2008;11:333-338

[27] Holsheimer J. Effectiveness of spinal cord stimulation in the management of chronic pain: analysis of technical drawbacks and solutions. Neurosurgery. 1997;40:990-999.

[28] Barolat G, Zeme S, Ketcik B. Multifactorial analysis of epidural spinal cord stimulation Stereotact. Funct. Neurosurg. 1991;56:77-103.

[29] Holsheimer J. Which neuronal elements are activated directly by spinal cord stimulation Neuromodulation. 2002;5:25-31.

[30] Computer modeling of spinal cord stimulation for low back pain. White paper prepared by Medtronic. 2007. 
Triple lead transverse tripoles 
Chapter 3 


\title{
CHAPTER 4
}

\section{Staggered transverse tripoles with quadripolar lateral anodes using percutaneous and surgical leads in SCS}

\author{
Vishwanath Sankarasubramanian, Jan. R. Buitenweg, Jan Holsheimer, Peter Veltink \\ MIRA, Institute for Biomedical Technology and Technical Medicine, University of Twente, Enschede, \\ The Netherlands
}

Accepted in Neurosurgery, doi: 10.1227, November 2012 


\begin{abstract}
Background: In Spinal Cord Stimulation (SCS) for low-back pain, the use of electrode arrays with both low power requirements and selective activation of target dorsal column (DC) fibers is desired. The Aligned Transverse Tripolar Lead (TTL) configuration offers the best DC selectivity. Electrode alignment of the same configuration using three parallel percutaneous leads is possible, but compromised by longitudinal migration resulting in loss of DC selectivity. This loss might be repaired by using the adjacent anodal contacts on the lateral leads.
\end{abstract}

Objective: To investigate if stimulation using adjacent anodal contacts on the lateral percutaneous leads of a staggered transverse tripole can restore DC selectivity. The effect of contact spacing and insulation is determined by comparing the performance of the above configuration with the same realized on a commercial, 3-column surgical lead.

Methods: Staggered transverse tripoles with quadripolar lateral anodes were modeled on the low-thoracic vertebral region (T10-T12) of the spinal cord using (a) PERC QD and (b) LAM QD, of same contact dimensions. The commercial LAM 565 surgical lead having 16 widely-spaced contacts was also modeled. For comparison with PERC QD, staggered transverse tripoles with dual lateral anodes were modeled using PERC ST.

Results: The PERC QD improved the depth of DC penetration and enabled selective recruitment of DCs as compared to PERC ST. Medio-lateral selectivity of DCs could not be achieved with the LAM 565.

Conclusion: Stimulation using PERC QD improves anodal shielding of DRs and restores DC selectivity._Based on our modeling study, we would hypothesize that, in clinical practice, LAM QD can provide an improved performance compared to the PERC QD. Our model also predicts that the same configuration realized on the commercial LAM 565 surgical lead with widely-spaced contacts cannot selectively stimulate DCs essential in treating low-back pain.

Key words: Paresthesia, Percutaneous leads, Spinal cord stimulation, Surgical leads, Transverse tripole, Usage range. 


\subsection{Introduction}

Spinal cord stimulation (SCS) is a minimally-invasive technique used in neuropathic pain modulation via stimulation of spinal dorsal column (DC) fibers (1-5). SCS is accompanied by a tingling sensation called paresthesia in the corresponding dermatomes. An important condition for analgesia is that the stimulation induced paresthesia covers the pain area completely $(1,6,7)$. SCS is effective, especially if complex, multidermatomal pain is to be treated as it provides a relatively easy accessibility of the spine and representation of all dermatomes caudal to the level of implantation (8). However, not all dermatomes can be easily captured by SCS. Areas which are hard to cover with paresthesia include most parts of the trunk, and low, mid and upper back (9). The low-back is a challenging target for stimulation due to multiple contributing factors, most important being the deep and medial position of low-back sensory fibers within the DCs and the high power required to stimulate these fibers (9-12).

Literature suggests that the most effective lead placement for low-back pain is a single midline lead or two leads straddling the midline placed at T8-T10 (8,13-16).A transverse tripolar arrangement is another option for capturing dermatomes in the low-back (17), although this technique currently requires placement of three parallel percutaneous leads or a surgical lead. The Transverse Tripolar configuration on a surgical lead (TTL), in combination with a dual-channel pulse generator is able to preferentially stimulate segments of spinal cord DCs combined with anodal shielding of DRs on either side (9,18-20). Such a selective and confined DC recruitment is most likely to help capture the dermatomes of the lower back.

Computer modeling studies with transverse tripoles on three parallel percutaneous leads (figure 1(A)) have shown to allow selective targeting of medial DC fibers, but only if the anodal and cathodal contacts are aligned (21). Unsuccessful alignment or migration of leads in the longitudinal direction resulted in a broad and bilateral DC activation, similar to that obtained with monopolar and longitudinal guarded cathode configurations (21). Such a wide DC area is believed to be recruited due to the diminished shielding of the DRs by the staggered anodes of the transverse tripole (figure 1(B)). Clinically, a wide DC recruitment represents a loss of medial DC fiber selectivity and hence potentially an absence of low-back paresthesias. This loss might be repaired by using the adjacent anodal contacts that are present on the lateral percutaneous leads (figure $1(\mathrm{C})$ ). This computer modeling study aims to primarily investigate if stimulation using the additional adjacent anodal contacts on the lateral percutaneous leads of a staggered transverse tripole can improve DR shielding and thereby restore selectivity of DC fibers, essential for treating low-back pain. 


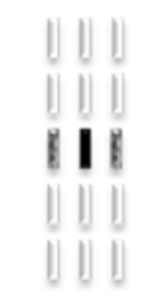

PERC AL

(A)

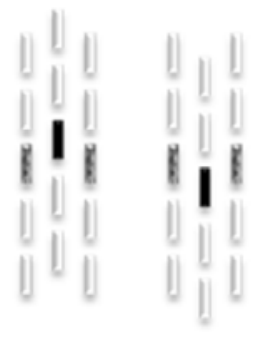

PERC ST $_{C}$ PERC ST $_{R}$

(B)

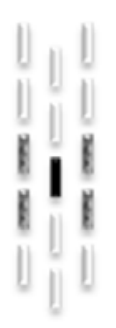

PERC QD

(C)

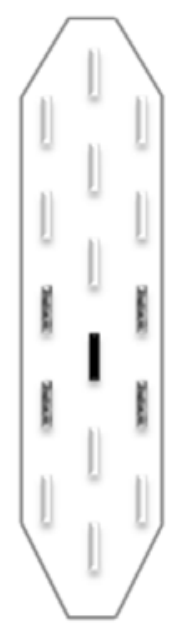

LAM 565

(D)

Figure 1 (A) Aligned transverse tripoles using three percutaneous leads - PERC AL, (B) Staggered transverse tripoles - PERC ST (caudal) and PERC ST $_{R}$ (rostral), (C) Staggered transverse tripoles with additional (quadripolar) adjacent anodes on the lateral leads- PERC QD and (D) Commercially available LAM 565 lead configured with staggered quadripolar lateral anodal contacts which are staggered with respect to the central cathode. The anodes are hatched and the cathodes are filled in (A), (B), (C) and (D).

Staggered quadripolar lateral anodal contacts can also be configured on commercially available surgical leads. A representation of such a contact configuration is possible with the commercially available 16electrode, 3-column surgical lead (LAM 565); with widely-spaced electrode contacts (figure 1(D)). Clinical studies and case reports have shown that at low-thoracic levels, there is a difference in performance of percutaneous and surgical leads (22). Surgical leads were shown to be technically superior to percutaneous leads in the management of low-back pain, in that they demonstrated improved pain coverage by stimulation paresthesias and required lower stimulation power $(22,23)$. Moreover, surgical leads due to their insulated backing allows for a more efficient, unidirectional stimulation field and stable lead position having a low tendency to migrate. Such a lead seldomly exhibits similar stimulation characteristics to that of a percutaneous lead. Therefore, the secondary aim of this modeling study is to determine the effect of contact spacing and insulation by comparing the underlying DC recruitment areas and power consumptions of the staggered quadripolar lateral anodal percutaneous lead configuration with the same realized on a commercially available 3-column surgical lead with 16 widelyspaced contacts (LAM 565). 


\subsection{Methods}

In order to simulate the effects of SCS, the University of Twente Spinal Cord Stimulation software was used (24-26). This software permits the implementation of a three-dimensional volume conductor model of the spinal column, including electrode arrays and nerve fibers $(24,26)$. The model has been validated and was used to identify the most important parameters for stimulation as well as novel lead designs $(14,15,18,26,27)$.

\subsubsection{Volume conductor model}

The 3D inhomogeneous volume conductor model consists of a spinal cord model around vertebral levels T10-T12. Its transverse geometry is shown in figure 2 (left).

A low-thoracic segment is modeled since it is the common spinal level for SCS implants. The model comprises Gray Mater (GM), White Mater (WM), Cerebro-Spinal Fluid (CSF), Dura Mater (DM), Epidural Space (ES) and a low conductivity layer around the ES. This layer represents the peripheral parts, like the vertebral bone, muscle, fat and skin. The geometries and electrical conductivities of these human anatomical structures were obtained from earlier modeling studies (26). The values of the DM and the surrounding layer were adjusted to match lead contact impedance data (28). Thickness of the dorsomedial CSF layer (dCSF) was $3.2 \mathrm{~mm}$, which is the mean value at the T10-T12 vertebral level (18). The conductivities of all the compartments are summarized in figure 2 (right). Except for the WM, all conductivities are isotropic. 


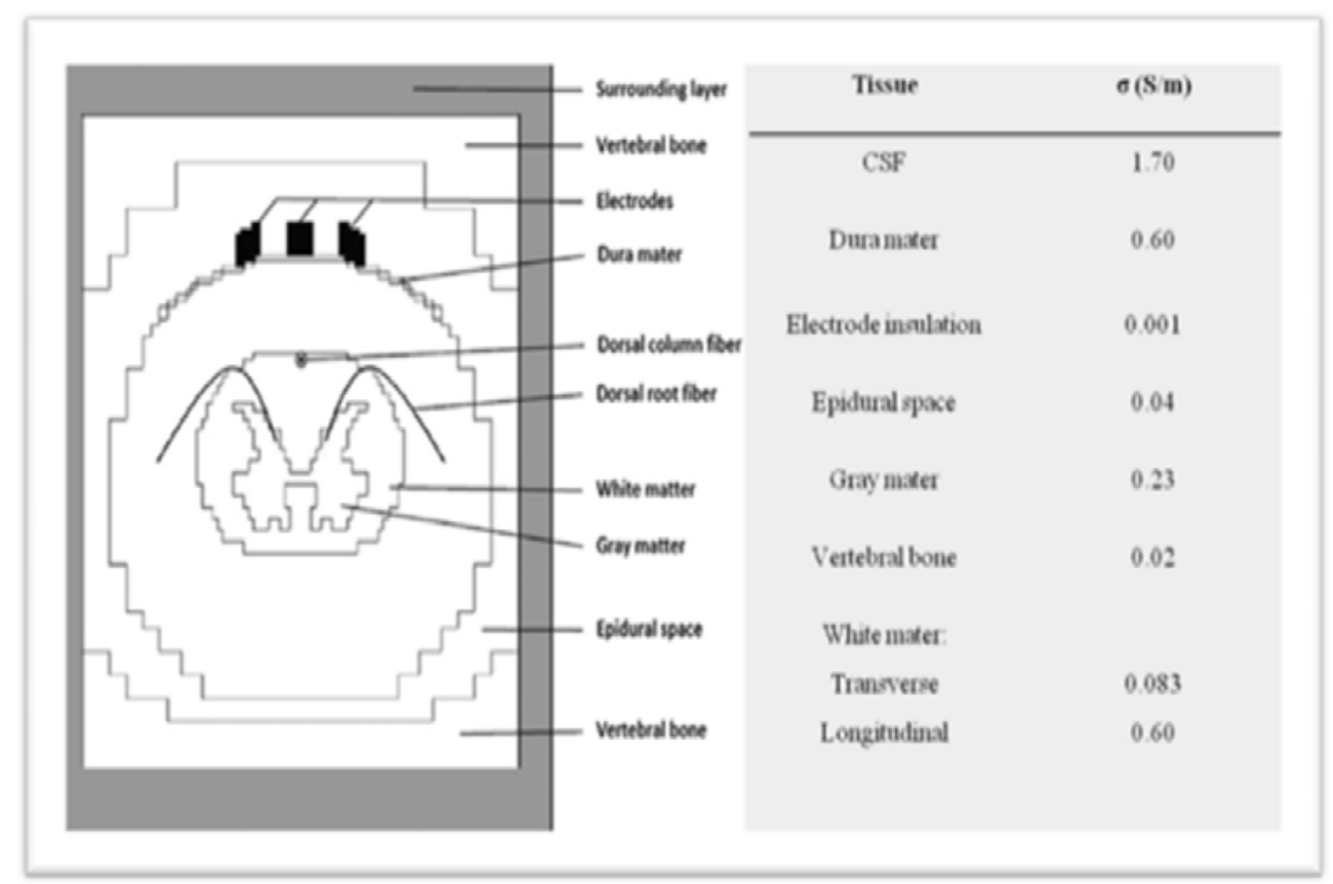

Figure 2 Left: Transverse section of the low-thoracic UT-SCS model of the spinal cord with the volume conductor elements, electrodes and nerve fibers. The grids are not depicted. Right: The conductivities of the volume conductor elements/tissues.

\subsubsection{Percutaneous and surgical lead types}

The 3D volume conductor model of the UT-SCS software includes the leads placed dorso-medially in the epidural space for electrical stimulation. Transverse tripolar configurations with quadripolar anodes staggered with respect to the central cathode were modeled using surgical and percutaneous leads.

Percutaneous lead with staggered quadripolar lateral anodal configuration -PERC QD. This percutaneous triple lead configuration modeled on the low-thoracic vertebral region of the spinal cord consists of five contacts in the transverse plane, namely a central cathode and four lateral anodes (two anodes on each side). The length of the contacts is $3 \mathrm{~mm}$ and the width and height of the contacts are 1 mm each. The centre lead with the cathode is placed over the spinal cord midline. The two lateral leads are offset longitudinally by $2 \mathrm{~mm}$ with respect to the centre lead. The anodes on the lateral leads are separated by a longitudinal edge-to-edge distance of $1 \mathrm{~mm}$. The lateral leads are placed medio-laterally at an edge-to-edge distance of $1.5 \mathrm{~mm}$ from the centre lead. The transverse steering span of the DCs represents the distance between the centres of the outer electrodes, and is $5 \mathrm{~mm}$. The configuration is abbreviated PERC QD (figure 3(A)). 
Laminotomy/Surgical lead with staggered quadripolar lateral anodal configuration-LAM QD. For this surgical lead configuration, the back of the electrode (dorsal structures) is insulated. This lead configuration is abbreviated $L A M Q D$ and is dimensionally similar to the PERC QD mentioned above (figure 3(B)). The total width of the surgical lead paddle is $7 \mathrm{~mm}$.

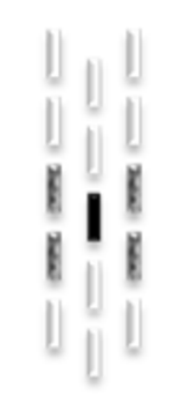

PERC QD

(A)

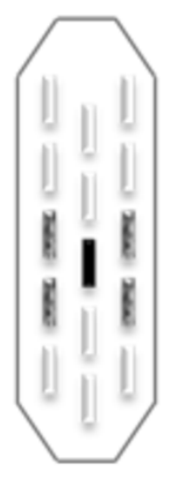

LAM QD

(B)

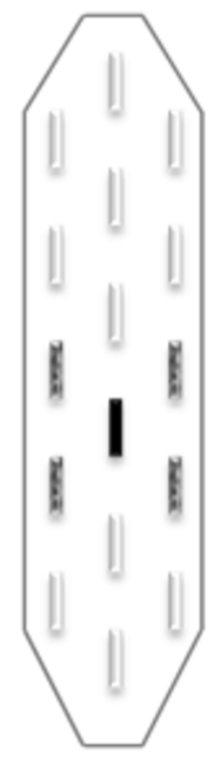

LAM 565

(C)

Figure $32 D$ projections of the lead models (A) PERC QD configuration. The lateral leads are longitudinally offset with respect to the central lead. (B) The LAM QD configuration. For both (A) and (B), the contact width and length is $1 \mathrm{~mm}$ and $3 \mathrm{~mm}$ respectively. The longitudinal edge-to-edge spacing between the contacts is $1 \mathrm{~mm}$. (C) The LAM 565 configuration. The contact length is $4 \mathrm{~mm}$ and the contact width is $1.5 \mathrm{~mm}$. The longitudinal edge-to-edge spacing between the contacts is $5 \mathrm{~mm}$. The anodes are hatched and the cathodes are filled in $(A),(B)$ and $(C)$.

Laminotomy/Surgical lead with widely-spaced staggered quadripolar lateral anodal configuration-LAM 565. A lead with a larger lead dimension as compared to the LAM QD configuration is modeled and simulated. This modeled lead configuration mimics the commercially available 16-electrode, 3-column surgical lead and is abbreviated LAM 565. The length of the contacts is $4 \mathrm{~mm}$ and the width is $1.5 \mathrm{~mm}$. The longitudinal edge-to-edge distance between the anodal contacts is $5 \mathrm{~mm}$, which is 5 times larger as compared to the modeled $L A M Q D$. The transverse steering span of the DCs is $6 \mathrm{~mm}$. The total width of the lead paddle is $10 \mathrm{~mm}$ (figure 3(C)). 
In all the lead configurations, both percutaneous and surgical, the lead position was always symmetrical with respect to the spinal cord midline. The spinal cord midline in our model coincides with the radiological midline. The leads were placed just behind the DM, which is generally the most favourable position in clinical applications.

\subsubsection{Nerve fiber model}

The fibers are modeled according to McNeal with modifications as described by Wesselink et al. (20,29). The fiber models used are compartmental models whose geometrical and electrical characteristics were chosen such that behaviour similar to that of human sensory fibers is mimicked (20). The myelinated nerve fiber model was defined at its anatomical position and its response to the applied field was calculated using potentials at the positions of its Ranvier nodes, obtained by interpolation of the grid potentials.

DC and DR nerve fibers which are part of the same primary afferent system were positioned in the volume conductor model. In comparison with the longitudinal DC fibers, the predominant features of the DR fibers are their curved shape and different orientation with respect to the spinal cord and the electrodes. The dimensions of the DC and DR fibers used in this study were obtained from Sankarasubramanian et al. 2011 (21).

\subsubsection{Stimulation parameters}

Transverse current-controlled electrical field steering was modeled by varying the anodal current ratio in several steps: 100/0, 90/10, 80/20, 70/30, 60/40, 50/50, 40/60, 30/70, 20/80, 10/90 and 0/100. These numbers represent the percentage of anodal current applied to the right lead anodes and to the left lead anodes respectively. For the two anodes in the same lead, the current is distributed equally. The authors arbitrarily assumed the pulse width to be $210 \mu \mathrm{sec}$, since in clinical studies, the average pulse width ranges from 175-600 $\mu \mathrm{sec}$ (30).

\subsubsection{Output parameters}

To enable a quantitative comparison of the performance of the modeled percutaneous and surgical lead types, several model output parameters were calculated. These parameters are defined below.

- $\mathrm{I}_{\mathrm{DC}}(\mathrm{mA})$ : DC fiber threshold, corresponding to the lowest activation threshold among all DC fibers.

- $\mathrm{I}_{\mathrm{DR}}(\mathrm{mA})$ : DR fiber threshold, corresponding to the lowest activation threshold of the DR fiber. It is calculated as the minimum of left DR fiber threshold $\left(\mathrm{I}_{\mathrm{DR}, \mathrm{L}}\right)$ and right $\mathrm{DR}$ fiber threshold $\left(\mathrm{I}_{\mathrm{DR}}\right.$, R). 
- $\mathrm{I}_{\mathrm{PT}}(\mathrm{mA})$ : Paresthesia threshold, corresponding to the current required to activate the lowest threshold fiber, being either a DC or DR fiber. Hence, it is calculated as the lowest value between $\mathrm{I}_{\mathrm{DC}}$ and $\mathrm{I}_{\mathrm{DR}}$.

- $\mathrm{I}_{\mathrm{DT}}$ (mA): Discomfort threshold, corresponding to the current at initial stimulation of proprioceptive DR fibers. It is defined as $\mathrm{I}_{\mathrm{DT}}=1.4 * \mathrm{I}_{\mathrm{DR}}$ (27).

- $\quad U R:$ Usage range, defined as the ratio between $\mathrm{I}_{\mathrm{DT}}$ and $\mathrm{I}_{\mathrm{PT}}$. $\mathrm{UR}=\mathrm{I}_{\mathrm{DT}} / \mathrm{I}_{\mathrm{PT}}$.

- $\mathrm{S}_{\mathrm{RA}}\left(\mathrm{mm}^{2}\right)$ : Maximum recruited DC area, which is the area between the dorsal border of the DCs and the recruitment contour at $\mathrm{I}_{\mathrm{DT}}$.

- W (mm): Width of recruited area, corresponding to the maximum medio-lateral extent of the recruited DC area at $\mathrm{I}_{\mathrm{DT}}$. It is also referred to as the span of DC recruitment.

- D (mm): Depth of recruited area, corresponding to the maximum dorso-ventral extent of the recruited DC area at $\mathrm{I}_{\mathrm{DT}}$.

- $\mathrm{Z}_{\mathrm{TIS}}(\mathrm{Ohm})$ : Tissue impedance, between the anodes and the cathodes. $\mathrm{Z}_{\mathrm{TIS}}=\mathrm{Z}_{\text {Cathode }}+0.25 \mathrm{Z}_{\text {Anode }}$ for a transverse tripolar configuration with quadripolar anodes. $\mathrm{Z}_{\text {Cathode }}$ and $\mathrm{Z}_{\text {Anode }}$ are the impedances at the cathode and the anode respectively.

- $\mathrm{Z}_{\mathrm{W}}(\mathrm{Ohm})$ : Wire impedance between the lead contact and the stimulator output. The $\mathrm{Z}_{\mathrm{W}}$ of the commercially available PERC QD and LAM 565 are $3 \mathrm{Ohm}$ and $36 \mathrm{Ohm}$ respectively. The $\mathrm{Z}_{\mathrm{W}}$ of the LAM QD was arbitrarily assumed to be 3 Ohm.

- $\mathrm{Z}_{\mathrm{G}}(\mathrm{Ohm})$ : Load or Total impedance, seen between the stimulator outputs. $\mathrm{Z}_{\mathrm{G}}=\mathrm{Z}_{\mathrm{TIS}}+1.25 \mathrm{Z}_{\mathrm{W}}$ for a transverse tripolar configuration with quadripolar anodes.

- $\quad \mathrm{E}_{\mathrm{DT}}(\mu \mathrm{J})$ : Energy per pulse at $\mathrm{I}_{\mathrm{DT}}$ delivered by the stimulator. It is calculated as $\mathrm{E}_{\mathrm{DT}}=\mathrm{I}_{\mathrm{DT}}{ }^{2} * \mathrm{Z}_{\mathrm{G}}$ * $\mathrm{T}$ (Equation 1), where $\mathrm{T}$ is the pulse width, being $210 \mu$ sec in all simulations.

Transverse tripolar stimulation enabled the estimation of $I_{D C}$ and $I_{D R}$ at various anodal current ratios. The area of recruited fibers in the DCs as well as the energy consumption was also calculated.

\subsection{Results}

\subsubsection{Transverse tripoles using percutaneous leads}

The lowest activation threshold among fibers placed near the dorsal border of the DCs $\left(\mathrm{I}_{\mathrm{DC}}\right)$ is plotted in figure 4 (Left) at various anodal steering ratios for the percutaneous triple lead transverse tripolar configurations (PERC AL, PERC ST (caudal), PERC ST $_{R}$ (rostral) and PERC QD). 

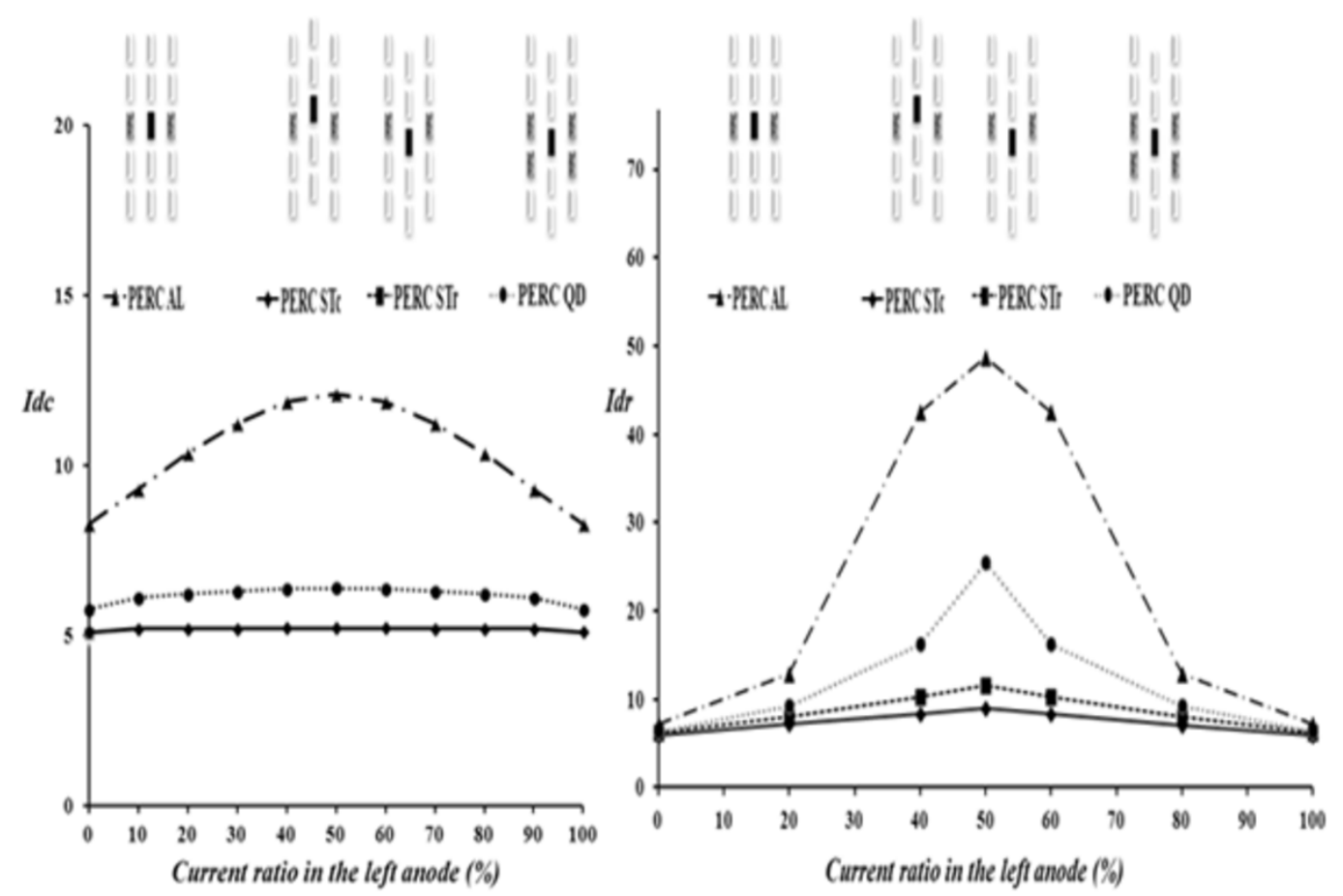

Figure 4 (Left) $I_{D C}$ and (Right) $I_{D R}$ for all the four transverse tripolar configurations on percutaneous leads as a function of the percentage of current applied to the left anode. In (A) the curves of the rostral and caudal anodes overlap and hence is seen as one curve. As a result there are three curves instead of four.

The $I_{D R}$ which corresponds to the minimum of the left and the right DR fiber thresholds is plotted in figure 4 (Right) at all steering ratios for the same lead configurations. The $\mathrm{I}_{\mathrm{DC}}$ and $\mathrm{I}_{\mathrm{DR}}$ values of the PERC QD were higher than PERC ST configurations but lower than the PERC AL configuration.

Highest $\mathrm{E}_{\mathrm{DT}}$, but highest medial DC selectivity was achieved with the PERC AL (figure 5(A)). Stimulation with PERC $\mathrm{ST}_{\mathrm{C}}$ and PERC $\mathrm{ST}_{\mathrm{R}}$ enabled a wide and bilateral DC recruitment at very low $\mathrm{E}_{\mathrm{DT}}$ (figure 5 (B) and $5(\mathrm{C})$ ) respectively. Deep penetration of the medial DCs was regained by stimulation with the PERC QD (figure 5 (D)), as compared to the PERC ST $\mathrm{C}_{\mathrm{C}}$ and PERC ST $\mathrm{R}_{\mathrm{R}}$. Also, a depth of DC recruitment similar to the PERC AL was attained with the PERC QD at a comparatively lower $\mathrm{E}_{\mathrm{DT}}$ (figure 5 (D)). 
(A)

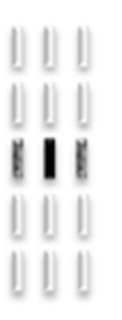

(B)

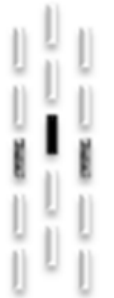

(C)

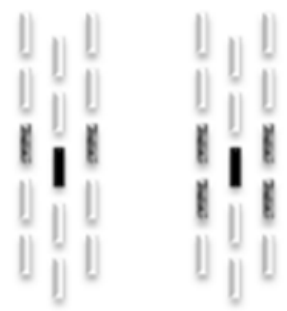

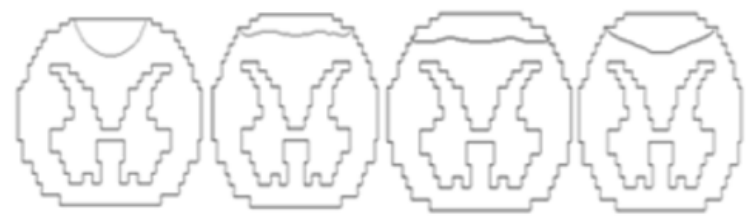

\begin{tabular}{|c|c|c|c|c|}
\hline Parameters & $\begin{array}{c}\text { PERC AL } \\
(\text { A) }\end{array}$ & $\begin{array}{c}\text { PERC ST } \\
\text { (B) }\end{array}$ & $\begin{array}{c}\text { PERC ST }_{\text {R }} \\
\text { (C) }\end{array}$ & $\begin{array}{c}\text { PERC QD } \\
\text { (D) }\end{array}$ \\
\hline W (mm) & 3.9 & 6.8 & 7.5 & 6.5 \\
\hline D (mm) & 1.3 & 0.7 & 0.9 & 1.3 \\
\hline $\mathrm{S}_{\mathrm{RA}}\left(\mathrm{mm}^{2}\right)$ & 3.8 & 3.5 & 5.2 & 6.0 \\
\hline $\mathrm{E}_{\mathrm{DT}}(\mu \mathrm{J})$ & 9.6 & 5.8 & 6.6 & 6.1 \\
\hline UR & 5.4 & 2.4 & 3.1 & 5.6 \\
\hline
\end{tabular}

Figure 5 Top: Recruitment contours in the DCs for all the four transverse tripolar configurations on percutaneous leads at $I_{D T}$. (A) Transverse tripoles with aligned anodes - PERC AL (B) Transverse tripoles with caudal anodes - PERC ST $C(C)$ Transverse tripoles with rostral anodes - PERC ST $T_{R}(D)$ Transverse tripoles with quadripolar anodes - PERC QD. Bottom: Width (W), Depth (D), maximum DC recruited area $\left(S_{R A}\right)$, energy at $I_{D T}\left(E_{D T}\right)$ and usage range (UR) for the same configurations.

\subsubsection{Transverse tripoles using surgical leads}

The calculated impedances, $\mathrm{E}_{\mathrm{DT}}$, UR and $\mathrm{S}_{\mathrm{RA}}$ at $\mathrm{I}_{\mathrm{DT}}$ for transverse tripolar stimulation with staggered quadripolar anodes configured on surgical leads is shown in figure 6(B) for LAM QD and 6(C) for LAM 565 respectively. For comparison, the same parameters for PERC QD are also depicted in figure 6(A).

\section{Comparison of PERC QD and LAM QD configurations.}

Transverse tripolar stimulation with the LAM QD recruits only a $5.3 \%$ larger and a $4.5 \%$ deeper DC area than the PERC QD at $\mathrm{I}_{\mathrm{DT}}$. The UR is 10.4 \% larger when stimulated with the LAM QD (figure 6(A) and 6(B)). The LAM QD configuration is therefore predicted to have a slightly better performance than the PERC QD configuration. 
(A) $\quad$ (B) $\quad$ (C)

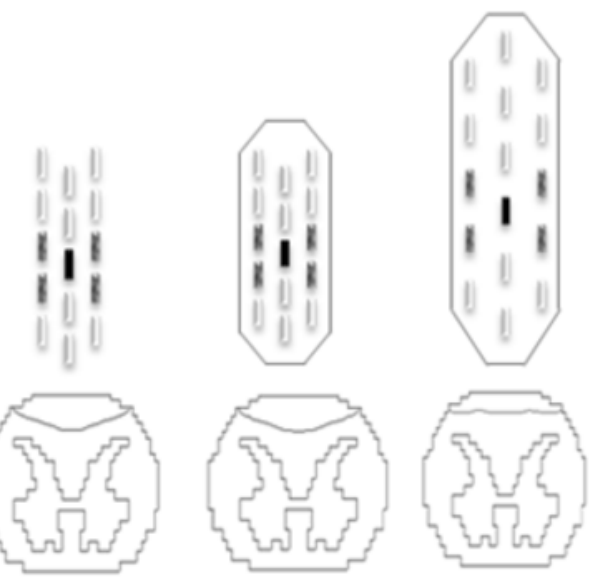

\begin{tabular}{|c|c|c|c|}
\hline Lead type & $\begin{array}{c}\text { PERC QD } \\
(\mathbf{A})\end{array}$ & $\begin{array}{c}\text { LAM QD } \\
(\mathbf{B})\end{array}$ & $\begin{array}{c}\text { LAM 565 } \\
(\mathbf{C})\end{array}$ \\
\hline $\mathrm{I}_{\mathrm{DT}}(\mathrm{mA})$ & 10.7 & 10.5 & 6.6 \\
\hline $\mathrm{Z}_{\mathrm{it}}(\mathrm{Ohm})$ & 249 & 299 & 215 \\
\hline $\mathrm{Z}_{\mathbb{W}}(\mathrm{Ohm})$ & 3 & 3 (assumed) & 36 \\
\hline $\mathrm{Z}_{\mathrm{O}}(\mathrm{Ohm})$ & 253 & 303 & 260 \\
\hline $\mathrm{E}_{\mathrm{DT}}(\mu \mathrm{\mu J})$ & 6.1 & 7.0 & 2.4 \\
\hline $\mathrm{UR}^{\mathrm{U}}$ & 5.6 & 6.2 & 2.5 \\
\hline $\mathrm{S}_{\mathrm{RA}}\left(\mathrm{mm}^{2}\right)$ & 6 & 6.3 & 4.1 \\
\hline
\end{tabular}

Figure 6 Top: Recruitment contours in the DCs with, (A) PERC QD (B) LAM QD and (C) LAM 565. Bottom: Calculated impedance values $\left(Z_{T i s}\right.$ and $\left.Z_{G}\right)$, energy consumptions $\left(E_{D T}\right)$, Usage Ranges (UR) and maximum DC recruited areas $\left(S_{R A}\right)$ for the same configurations.

The $\mathrm{Z}_{\text {Tis }}$ as seen by the contacts and $\mathrm{Z}_{\mathrm{G}}$ for the respective configurations are summarized in figure 6(A) and 6(B). The $\mathrm{I}_{\mathrm{DT}}$ is also indicated. As shown, the surgical lead has a higher $\mathrm{Z}_{\text {Tis }}$ than the percutaneous lead having the same transverse tripolar contact configuration and dimension, i.e the $\mathrm{Z}_{\text {Tis }}$ of LAM QD is higher than the $\mathrm{Z}_{\text {Tis }}$ of PERC QD. Assuming that the $\mathrm{Z}_{\mathrm{W}}$ of the LAM QD is the same as the PERC QD (3 Ohms), the total impedance $\left(\mathrm{Z}_{\mathrm{G}}\right)$ of the LAM QD is relatively higher than that of the PERC QD. Also, the $I_{D T}$ is slightly lower for LAM QD than for the PERC QD. Higher $E_{D T}$ is consumed when a LAM QD is used for stimulation (figure 6B). 


\section{Comparison of LAM QD and LAM 565 configurations.}

From figure 6(B) and 6(C) it is seen that, the $S_{R A}$ is larger $\left(6.3 \mathrm{~mm}^{2}\right)$ when stimulated with the LAM QD configuration than with that of the commercial LAM 565 configuration $\left(4.1 \mathrm{~mm}^{2}\right)$. Also, the LAM QD achieves the largest UR (6.2) and depth of DC recruitment (1.4 mm). However, it also requires the highest $\mathrm{E}_{\mathrm{DT}}\left(\mathrm{E}_{\mathrm{DT}}=7.0 \mu \mathrm{J}\right)$.

Since the contact area of the commercial LAM $565[4 *(4 \mathrm{~mm} * 1.5 \mathrm{~mm})]$ is larger than the LAM QD [4 *(3 mm* $1 \mathrm{~mm})$ ] and PERC QD [4*(3 mm*1 mm)], its $\mathrm{Z}_{\text {Tis }}$ is substantially lower. Due to a sufficiently large $Z_{W}(36 \mathrm{Ohm})$ of the LAM 565, its total impedance $\left(Z_{G}\right)$ is considerably larger than its $Z_{\text {Tis }}$. Overall, the LAM 565 had the lowest $\mathrm{E}_{\mathrm{DT}}(2.4 \mu \mathrm{J})$ as compared to the LAM QD and the PERC QD (figure 6).

(A)

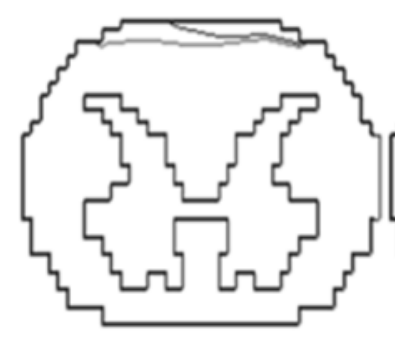

(B)

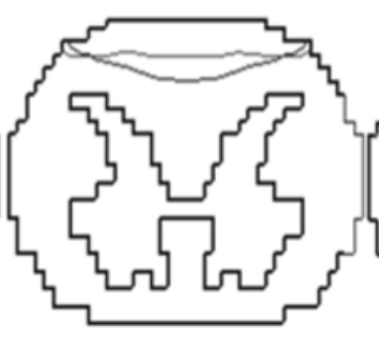

(C)

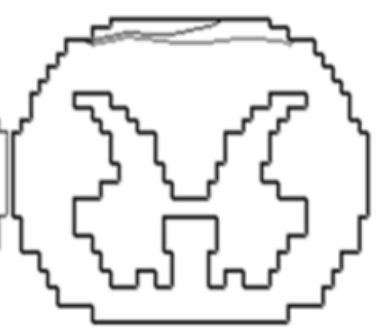

Figure 7 Recruitment contours indicating the ventral boundary of the $D C$ area at $I_{D T}$ for the $L A M Q D$ (black line) and the LAM 565 (grey line) configurations at anodal current ratios of (A) 100/0, (B) 50/50 and (C) 0/100 \% respectively.

Figure 7 depicts the DC recruitment contours at $\mathrm{I}_{\mathrm{DT}}$ for the LAM QD and commercial LAM 565 configurations, at anodal current ratios of 0/100, 50/50 and 100/0 \% respectively. For the LAM QD configuration, the area of DC activation was bilateral (figure 7 (B)), when equal anodal currents were applied through the lateral anodes on either sides. When the anodal current was steered from the right lead (100/0 \%) to left lead (0/100 \%), the DC recruitment contour moved from left to right; that is the shift was towards the side having a decreased injection of anodal current (figure 7(A) and 7(C)).

Medio-lateral selectivity of the DCs could not be achieved with the commercial LAM 565 configuration (figure 7 (A-C)). The configuration predominantly recruited a wide and bilateral DC area. This also means that steering of anodal currents between the lateral leads of this configuration cannot target medially-confined populations of DCs in the spinal cord. 


\subsection{Discussion}

The primary aim of this computer modeling study was to investigate if stimulation using adjacent anodal contacts on the lateral percutaneous leads of a staggered transverse tripole can restore selectivity of DCs necessary for low-back pain relief. The secondary aim of this computer modeling study was to determine the effect of contact spacing and insulation by comparing the underlying DC recruitment areas and power consumptions of the quadripolar lateral anodal percutaneous lead configuration (PERC QD) with the same realized on the commercially available 3-column surgical lead with 16 widely-spaced contacts (LAM 565). The effects of staggered_quadripolar lateral anodal stimulation, contact insulation and longitudinal contact spacing are discussed in the three sections below.

\subsubsection{Stimulation using dual and quadripolar anodes}

Results showed that stimulation using the PERC QD configuration required higher currents for DC and DR fiber activation than that of PERC ST configurations (figure 4). The higher currents are needed because of the confined electric field elicited by the quadripolar anodes of the PERC QD as compared to dual anodes of the PERC ST. A higher $\mathrm{I}_{\mathrm{DR}}$ (figure 4, right) in particular, suggests an improved shielding of DR fibers by the adjacent anodal contacts on the lateral leads of the PERC QD configuration. The effect is manifested in the form of a reduced width of recruited DC area (figure 5D).

$I_{D R}, E_{D T}$ and medial DC selectivity is highest for the aligned configuration (PERC AL) among all the transverse tripolar percutaneous lead configurations (figure 4 right). $I_{D T}$ derives from $I_{D R}$ and thus the same observation holds for $\mathrm{I}_{\mathrm{DT}}$. Even though $\mathrm{I}_{\mathrm{DT}}$ was largest for the PERC AL, the usage range (UR), which takes both $\mathrm{I}_{\mathrm{PT}}$ and $\mathrm{I}_{\mathrm{DT}}$ into account, was the largest for the PERC QD (figure 5D).

A large UR is indicative of the fact that DR fibers have higher activation thresholds compared to DC fibers. Hence, greater penetration into the DCs with less lateral spread of current can be obtained without stimulating the nearby DR fibers. Rightly so, the deepest DC recruitment $(1.3 \mathrm{~mm})$ similar to the PERC AL was attained with the PERC QD configuration (figure 5D). This implies that, deep low-back sensory fibers in the medial DCs can be reached without DR activation. Compared to the PERC AL, the PERC QD is likely to be a better candidate for a clinically applicable contact configuration to stimulate low-back fibers; it can favor preferential activation of DCs vs. DRs for a large UR and lower $\mathrm{E}_{\mathrm{DT}}$, without causing adverse effects.

Unlike the PERC ST configurations, steering the anodal currents of the PERC QD from left to right enables right-left control of DC activation. Therefore, DC selectivity is restored by stimulation using the additional adjacent anodal contacts on the lateral percutaneous leads of the staggered transverse tripole. By restoring DC selectivity, the PERC QD configuration can be useful in treating low-back pain. 


\subsubsection{Effect of contact insulation - Comparison of PERC QD and LAM QD}

The modeling results showed that the LAM QD recruited only a slightly larger and deeper DC area than the PERC QD configuration. The LAM QD configuration also required higher energy for stimulation $\left(\mathrm{E}_{\mathrm{DT}}\right)$ as compared to the PERC QD (figures 6(A) and 6(B)).

The contacts of the LAM QD and PERC QD were modeled as a polygonal surface on an insulating paddle, and as four rectangular surfaces around an insulating square bar, respectively. Their dimensions were set to their respective contact areas of $12 \mathrm{~mm}^{2}$. The LAM QD differs from the PERC QD only because of its stable insulated backing at the dorsal side of the contact. The contacts of the PERC QD, on the other hand, are around the surface of the electrode with the current free to spread circumferentially. Therefore, the $\mathrm{Z}_{\text {Tis }}$ of the PERC QD is lower than the LAM QD (figures 6(A) and 6(B)). Since, the $\mathrm{Z}_{\mathrm{W}}$ of the LAM QD is assumed to be the same as the PERC QD, the difference in total/load impedance $\left(Z_{G}\right)$ of the configurations is similar to the respective differences in their tissue impedances $\left(\mathrm{Z}_{\text {Tis }}\right)$. Consequently, according to equation 1 , the energy per pulse $\left(\mathrm{E}_{\mathrm{DT}}\right)$ delivered by the stimulator is lower with the PERC QD than the LAM QD (figure 6(A)).

In clinical practice, the measured $\mathrm{Z}_{\mathrm{Tis}}$ and $\mathrm{E}_{\mathrm{DT}}$ of the PERC QD configuration will be higher than the values predicted above by computer modeling. In clinical scenario, due to a substantially more epidural space (ES) dorsally in the T10-T12 region, the likelihood of the PERC QD leads to have dural contact is less. Because of this, the dorso-ventral position of the leads may vary from a position just adjacent to the dura mater (DM) to a position more dorsal in the ES. Therefore, the PERC QD leads are more likely to have contacts surrounded by epidural fat, resulting in a higher $\mathrm{Z}_{\text {Tis }}$ and therefore higher measured $\mathrm{E}_{\mathrm{DT}}(8)$.

Our modeling results predicted the LAM QD configuration to have a slightly better performance than the PERC QD configuration. Only a 10.4 \% larger UR, 4.5 \% deeper DC penetration and 5.3 \% larger area of DC activation was achieved by stimulation with the LAM QD as compared to the PERC QD. These results are obtained without considering the reduction of dCSF due to the volume of the surgical lead paddle. Inclusion of the surgical lead paddle volume, as would occur in clinical scenarios volume would mean that, the LAM QD configuration positioned next to the DM will push the dura ventrally, reducing dCSF (8). The reduced dCSF indicates a smaller distance to the neural target and reduced stimulation thresholds. Because $I_{D C}$ is reduced more steeply than $I_{D R}$ when the distance is reduced, this will result in an increased UR and thus increased paresthesia coverage (15). Moreover the energy consumption is also reduced as compared to the PERC QD. Hence, in clinical practice, staggered quadripolar lateral anodes configured on a 3-column surgical lead (LAM QD) can provide an improved performance than the PERC QD in treating low-back pain complaints. 
The LAM QD is a hypothetical 3-column surgical lead modeled having the same staggered quadripolar lateral anodal configuration and contact dimensions as the PERC QD. Earlier clinical studies with surgical leads that reported results for low-back pain used single-column and dual-column surgical leads $(22,23,31)$. Bipolar contact combinations were configured on the leads. Besides, such leads have electrode contacts with larger active contact surface area, and that are widely-spaced as compared to the LAM QD. Therefore, comparison of the underlying DC recruitment areas and energy consumptions of the LAM QD with the widely-spaced single-column and dual-column surgical lead configurations is not relevant.

\subsubsection{Effect of contact spacing - Comparison of LAM QD AND LAM 565}

The commercial, 3-column LAM 565 surgical lead configuration, with widely-spaced contacts, required the lowest energy but also resulted in lowest UR (figure 6C). This is in general agreement with the earlier clinical trials that compared the performance of percutaneous and surgical leads $(22,23,31)$. Moreover, a small pilot study by Rigoard et al. showed that stimulation using the commercial LAM 565 lead can provide bilateral paresthesia coverage of the back in patients with Failed Back Surgery Syndrome (32). Our modeling study with the same configuration of LAM 565 shows that the ability to selectively recruit DC fibers is diminished as compared to the LAM QD (figure 7). The decreased DC selectivity and decreased performance of LAM 565 as compared to LAM QD is due to the large contact spacing between the anodes and the cathodes of the LAM 565. The LAM 565 configuration always enabled a wide and bilateral DC recruitment. This means that steering of anodal currents between the lateral leads of this configuration cannot target medially-confined populations of DCs in the spinal cord.

The commercial LAM 565 configuration has larger longitudinal contact spacing as compared to the LAM QD. The larger the contact spacing, the weaker is the influence of the anodes on the cathodal field, so that the performance of the tripole approaches the performance of the monopole in all respects. Larger contact spacing also resulted in a lower usage range (UR), indicative of decreased DC versus DR fiber selectivity (figure 6C). On the other hand, smaller contact spacing as in the LAM QD configuration resulted in an increase of the electric field due to superposition and a more effective stimulation of the DCs. Therefore, the LAM QD recruited more fibers medial to the electrode than the LAM 565 configuration. This implies that the main parameter determining DC fiber selectivity is the longitudinal contact spacing.

The lateral or transverse contact spacing of the 3-column transverse tripolar configuration is shown to minimally impact the DC fiber selectivity and hence was not modeled and discussed in this study (33). Tightly spaced contacts of a quadripolar lateral anodal configuration, staggered with respect to the midline cathode, increase the ability in regaining the medio-lateral steering capability in the DCs at the cost of large energy consumption. Definitely, a trade-off between therapeutic effect and energy saving has 
to be made. Increased energy consumption may be acceptable if it brings benefits for the patient, especially as the new generation of implantable pulse generators (IPG) has rechargeable batteries with yet a larger capacity than the former ones. This concept has not yet been validated through clinical studies. However, it is noteworthy that 3-column surgical leads configured as transverse tripoles with tightlyspaced staggered quadripolar lateral anodes make available to the clinician theoretically optimal combinations, which leads with widely-spaced contacts do not.

\subsection{Conclusions}

Computer modeling predicts that stimulation using the adjacent anodal contacts on the lateral percutaneous leads of a staggered transverse tripole (PERC QD) improves anodal shielding of DRs, allows deeper penetration in the DCs, and restores DC selectivity. Based on our modeling study, we would hypothesize that, in clinical practice, transverse tripoles with staggered quadripolar lateral anodes configured on a 3-column surgical lead (LAM QD) can provide an improved performance compared to the PERC QD in treating low-back pain complaints. The consequences in clinical practice partly were not described by the model. Our model also predicts that the same configuration realized on the commercial LAM 565 surgical lead with widely-spaced contacts cannot selectively stimulate DCs essential in treating low-back pain. To target medio-lateral populations of DC fibers in the spinal cord, all active contacts of the transverse tripole lead configuration should be closely-spaced.

\section{Acknowledgements}

The authors gratefully thank Boston Scientific Neuromodulation (Valencia, CA, USA) for their grant to support this research. 


\section{References}

[1] Barolat G. Spinal cord stimulation for chronic pain management. Arch. Med. Res. 2000;31:258-262.

[2] Oakley JC, Prager JP. Spinal cord stimulation: Mechanisms of action. Spine. 2002;27:2574-2583.

[3] Holsheimer J. Effectiveness of spinal cord stimulation in the management of chronic pain: analysis of technical drawbacks and solutions. Neurosurgery. 1997;40:990-999.

[4] Van Buyten JP. Neurostimulation for chronic neuropathic back pain in failed back surgery syndrome. Journal of Pain and Symptom Management. 2006;31(4 Suppl):S25-29.

[5] Alo KM, Redko V, Chamov J. Four year follow-up of dual electrode spinal cord stimulation for chronic pain. Neuromodulation. 2002;5:79-88.

[6] North RB, Ewend MG, Lawton MT and Piantadosi S. Spinal cord stimulation for chronic, intractable pain: superiority of 'multi-channel' devices. Pain. 1991;44:119-130.

[7] North RB. Spinal cord stimulation for chronic, intractable pain. Adv. Neurol. 1993;63:289-301.

[8] Manola L, Holsheimer J, Veltink P. Technical performance of percutaneous leads spinal cord stimulation: a modeling study. Neuromodulation. 2005;8:88-99.

[9] Oakley JC. Spinal cord stimulation in axial low back pain: solving the dilemma. Pain Medicine 7. 2006;S58-63.

[10] Holsheimer J. Does dual lead stimulation favor stimulation of the lower back? Neuromodulation. 2000;3:55-57.

[11] Barolat G, Zeme S, Ketcik B. Mapping of sensory responses to epidural stimulation of the intraspinal neural structures in man. Neurosurg. 1993;78:233-239.

[12] Stojanovic MP, Abdi S. Spinal cord stimulation. Pain physician. 2002;5:156-166.

[13] Law JD. Spinal stimulation: statistical superiority of monophasic stimulation of narrowly separated, longitudinal bipoles having rostral cathodes. Appl Neurophysiol. 1983;46:129-137.

[14] Holsheimer J, Struijk JJ, Tas NR. Effects of electrode geometry and combination on nerve fiber selectivity in spinal cord stimulation. Med Biol Eng Comput. 1995;33:676-682.

[15] Holsheimer J, Wesselink WA. Effect of anode-cathode configuration on paresthesia coverage in spinal cord stimulation. Neurosurgery. 1997;41:654-659.

[16] North RB, Kidd DH, Olin J. Spinal cord stimulation for axial low back pain: a prospective, controlled trial comparing dual with single percutaneous electrodes. Spine. 2005;30:1412-1418.

[17] Hegarty D. Spinal cord stimulation: The clinical application of new technology (Review article). Anesthesiology Research and Practice. 2011.

[18] Holsheimer J, Nuttin B, King GW, Wesseling WA, Gybels JM, de Slutter P. Clinical evaluation of paresthesia steering with a new system for spinal cord stimulation. Neurosurgery. 1998;42:541-549. 
[19] Struijk JJ, Holsheimer J. Transverse tripolar spinal cord stimulation: theoretical performance of a dual channel system. Med. Biol. Eng. Comput. 1996;34:273-279.

[20] Wesselink WA, Holsheimer J, King GW, Torgerson NA, Boom HBK. Quantitative aspects of the clinical performance of transverse tripolar spinal cord stimulation. Neuromodulation. 1999; 2:5-14.

[21] Sankarasubramanian V, Buitenweg JR, Holsheimer J, Veltink P. Electrode alignment of transverse tripoles using a percutaneous triple-lead approach in spinal cord stimulation. J. Neural Eng. 2011. doi:10.1088/1741-2560/8/1/016010.

[22] North RB, Kidd DH, Olin JC, Sieracki JM. Spinal cord stimulation electrode design: prospective, randomized, controlled trial comparing percutaneous and laminectomy electrodes: part 1 . Technical outcomes. Neurosurgery. 2002;51:381-390.

[23] North RB, Lanning A, Hessels R, Cutchis PN. Spinal cord stimulation with percutaneous and plate electrodes: side effects and quantitative comparisons. Neurosurg. Focus. 1997;2:1-5.

[24] Holsheimer J. Computer modeling of spinal cord stimulation and its contribution to therapeutic efficacy (review). Spinal Cord. 1998;36:531-540.

[25] Struijk JJ, Holsheimer J, van der Heide G, Boom HBK. Recruitment of dorsal column fibers in spinal cord stimulation: influence of collateral branching. IEEE Trans. Biomed. Eng. 1992;39:903-912.

[26] Struijk JJ, Holsheimer J, Boom HBK. Excitation of dorsal root fibers in spinal cord stimulation: a theoretical study. IEEE Trans. Biomed. Eng. 1993;40:632-639.

[27] He J, Barolat G, Ketcik B. Stimulation usage range for chronic pain management. Analgesia. 1995;1: 75-80.

[28] Alo K, Varga C, Krames E, Prager J, Bradley K. Variability of contact impedance by vertebral placement in spinal cord stimulation. Abstracts of the $54^{\text {th }}$ Congress of Neurological Surgeons San Fransisco, CA, October 16-2. 2005.

[29] McNeal DR. Analysis of a model for excitation of myelinated nerve. IEEE Trans. Biomed. Eng. 1976; 23:329-337.

[30] Kries PG, Fishman SM. Spinal cord stimulation: percutaneous implantation techniques. Oxford: Oxford University Press. 2009.

[31] Villavicencio A, Leveque J, Rubin L, Bulsara K, Gorecki J. Laminectomy versus percutaneous electrode placement for spinal cord stimulation. Neurosurgery. 2000;46:399-406.

[32] Rigoard P, Delmotte A, D’Houtaud S, Misbert L, Diallo B, Roy-Moreau A, Durand S, Royoux S, Giot JP, Bataille B. Back pain: a real target for spinal cord stimulation? Neurosurgery. 2012;70:574585.

[33] Wesselink WM, North RB. Three column contact patterns for Spinal cord stimulation offer selective dorsal column fiber activation. Neurosurgery. 2006;59:470-471. 
Chapter 4 


\title{
CHAPTER 5
}

\section{Performance of transverse tripoles vs longitudinal tripoles with anode intensification: computational modeling study}

\author{
Vishwanath Sankarasubramanian, Jan. R. Buitenweg, Jan Holsheimer, Peter Veltink \\ MIRA, Institute for Biomedical Technology and Technical Medicine, University of Twente, Enschede, \\ The Netherlands \\ Submitted in Neuromodulation
}




\begin{abstract}
Background: Spinal Cord Stimulation (SCS) is a reversible neurostimulation technique used to alleviate refractory chronic pain, by stimulation of large cutaneous fibers in the spinal dorsal columns (DCs). Recruitment of large cutaneous fibers is challenged either by direct electrical stimulation of DC fibers or by their stimulation via dorsal root (DR) activation. The latter limits the coverage of the pain area by paresthesia and also evokes discomfort sensations. In configurations where the anodes are placed longitudinally on both sides of the cathode (referred to as Longitudinal Guarded Cathodes, LGC) and are closely spaced, efficient recruitment of the DCs is achieved but at the cost of large energy consumption. Increase in center-to-center (C-C) spacing of LGCs affects both preferential stimulation and energy consumption. In fact this increase in $\mathrm{C}-\mathrm{C}$ mimics the change of a tripole towards monopoles. On the contrary, a small spacing step (LGC+), if included in the contact spacing of the commercially available LGC can be an efficient method to study the local effects around the electrode. Transverse tripolar stimulation (TTS), on the other hand, enables preferential activation of DC over DR fibers. The currents from the laterally placed anodes effectively shield the DRs from activation. Therefore, the anodes have a strong effect over the area of DC activation, both when configured longitudinally and transversally with respect to the cathode. These anodal functions might be exploited more effectively by increasing the intensity of anodal currents, but this has never been studied in detail.
\end{abstract}

Objective: The primary aim of this computer modeling study is to investigate if enhanced DC recruitment is achieved when anodal currents in transverse tripolar and longitudinal tripolar combinations (both LGC and LGC+) are increased up to 30\% with respect to the cathodal current. Secondly, the merits of anodal intensification (AI) are evaluated by comparing the DC recruitment areas and energy consumption of LGC+ with AI, against stimulation using an LGC without AI.

Methods: Using the UT-SCS computer modelling platform, the local effects around the commercially available LGC lead with $4.0 \mathrm{~mm}$ center-to-center (C-C) spacing was studied by LGC+ (LGC lead with a wider C-C spacing) modeled on a single percutaneous lead with $4.5 \mathrm{~mm} \mathrm{C-C}$. TTS was modeled on triple percutaneous leads. Electrical fields of TTS were compared against stimulation using LGC and LGC+, all combined with AI. The maximum DC recruited area $\left(\mathrm{S}_{\mathrm{RA}}\right)$, usage range (UR) and energy consumptions $\left(\mathrm{E}_{\mathrm{DT}}\right)$ were computed at discomfort thresholds $\left(\mathrm{I}_{\mathrm{DT}}\right)$ for the respective configurations.

Results: TTS with 10\% AI recruited a smaller depth and width of medial DC recruited area as compared to the situation with no AI. In contrast, AI of LGC and LGC+ resulted in increasing the depths and widths of the recruited DC area respectively. Also, AI of LGC+ recruited a larger $S_{R A}$ and UR compared to that 
of LGC without AI. The additional $\mathrm{S}_{\mathrm{RA}}$ and UR of LGC+ with AI were achieved at lower $\mathrm{E}_{\mathrm{DT}}$ than that of LGC without AI.

Conclusions: AI of TTS is not advantageous, as the same DC recruitment can be achieved at lower stimulation amplitudes with balanced anodal and cathodal currents. LGC and LGC+ with AI allow additional DC stimulation, which may increase the likelihood of activating fibers inaccessible with conventional programming. LGC+ with AI can be more efficient than LGCs without AI, as a larger $\mathrm{S}_{\mathrm{RA}}$ and UR is achieved at lower energy expenditure. 
Chapter 5 


\subsection{Introduction}

Spinal cord stimulation (SCS) is a neurostimulation technique used to alleviate refractory chronic pain, via stimulation of spinal dorsal column (DC) fibers among other neuronal pathways $(1,2)$. Successful pain relief is attributed to a combination of factors, the most important being the paresthesia coverage of the targeted pain area (2,3-5). In SCS, an array of stimulating contacts placed in the dorsal epidural space is programmed to perform as either anodes or cathodes. The anodal currents that are injected in a longitudinal or transverse fashion with respect to the cathode, determine what DC structures need to be activated (6). It is shown that in comparison to the stimulation field in the DCs evoked by longitudinal tripolar configurations, transverse tripoles (TT) create a field deeper in the DCs but confined to its median part (7-10). In order to enhance the DC recruitment without stimulating the lateral dorsal roots (DR), an additional anodal current might be necessary. Can the additional injected anodal current enable deeper DC stimulation as compared to configurations that use conventional programming?

Stimulation of spinal DC fibers is essential for obtaining broad paresthesia coverage (11). Both computer modeling studies and clinical trials have shown that the DCs of the spinal cord are most efficiently stimulated by longitudinal tripolar configuration placed on the physiological spinal cord midline (4). In such a configuration, the longitudinally placed anodes with respect to the cathode modulate the stimulation field, and hence the recruitment of DC fibers. The main current component of this stimulation field corresponds to the orientation of DC fibers, thereby enabling efficient DC activation. Mathematical modeling has also highlighted the potential benefits of small center-to-center (C-C) contact spacing in longitudinal tripoles. The tripoles perform better when configured with small C-C spacing as Longitudinal Guarded Cathodes (LGC). Large paresthesia coverage is obtained when LGCs are used. Stimulation with LGCs and improved paresthesia coverage is accompanied by increased energy consumption $(12,13)$. Earlier studies have also shown that an increase in C-C spacing of LGCs affects both preferential stimulation and energy consumption $(11,12,14)$. Recognizing the effects of C-C contact spacing, and knowing that an increase in the spacing mimics the change of a tripole towards monopoles, a small spacing step is modelled in the LGC configuration (LGC+). This small spacing step can be an efficient method to study the local effects around the commercially available LGC electrode.

Also, stimulation of DC fibers is challenged by recruitment of DR fibers. In fact this increase in C-C mimics the change of a tripole towards monopoles. On the contrary, a small spacing step (LGC+), if included in the contact spacing of the commercially available LGC can be an efficient method to study the local effects around the electrode. DR stimulation evokes paresthesias only in one to two dermatomes. Also, recruitment of reflex motor nerve fibers intermingled among the sensory nerve fibers of the DR can lead to patient discomfort. The above effects can hinder optimum paresthesia coverage of the pain area. 
Optimized programming studies and clinical trials $(7,8,15)$ have shown that DR activation can be reduced by a concept termed anodal shielding. In other words, the anodes, if placed laterally with respect to the cathodes, practically function to prevent the DR structures from activation. Transverse Tripolar Stimulation (TTS) made use of this concept by injecting anodal currents from the transversally placed flanking anodes (7). The stimulating cathode is placed at the physiological midline of the spinal cord and is important to stimulate the desired DC fibers. Mathematical modeling has suggested that transverse tripolar (TT) configurations can recruit mostly deep medial fibers in the DCs before DR fibers start to limit the therapy.

In both TTS and longitudinal tripolar stimulation discussed above, the amount of injected cathodal and anodal currents is equal. Although the stimulation outcome is mostly determined by the cathode, the presence of nearby anodes modulates the stimulation field and hence the paresthesia coverage. By injecting an additional anodal current, a resulting net positive anodal current is obtained. Net cathodal currents can further be increased, for example, from the case of the implantable pulse generator (IPG) to recruit an additional volume in the DCs.

The primary aim of this computer modeling study is to investigate if enhanced DC recruitment is achieved when anodal currents in transverse tripolar and longitudinal tripolar (both LGC and LGC+) contact combinations are increased up to $30 \%$ with respect to the cathodal current. Secondly, the merits of anodal intensification (AI) are evaluated by comparing the DC recruitment areas and energy consumption of LGC+ with AI, against stimulation using a LGC without AI.

\subsection{Methods}

In order to evaluate the effects of the proposed Anode Intensification (AI) technique on the electrical field potential and the recruitment of nerve fibers, computer simulations were performed using the University of Twente - Spinal Cord Stimulation (UT-SCS) software $(16,17)$. Each model consisted of a 3D volume conductor, an array of lead contacts and nerve fiber models.

\subsubsection{Volume conductor model}

A 3D model of the low-thoracic vertebral region (T10-T12) of the spinal cord was used. The dimensions of the modeled anatomical compartments (spinal cord, dural sac, epidural space, etc.) were taken from an MRI study on spinal cross-sections (18). Thickness of the dorso-medial CSF layer (dCSF) was $3.2 \mathrm{~mm}$, which is the mean value at the T10-T12 vertebral level (8). A transverse cross-section of the model is shown in figure 1. Electrical conductivities of the compartments were obtained from Struijk et al. 1993 
(19) with modifications as described in Manola et al. 2004 (17). In order to obtain the stimulation-induced electrical potential field, a discretized form of the Laplace equation was solved numerically.

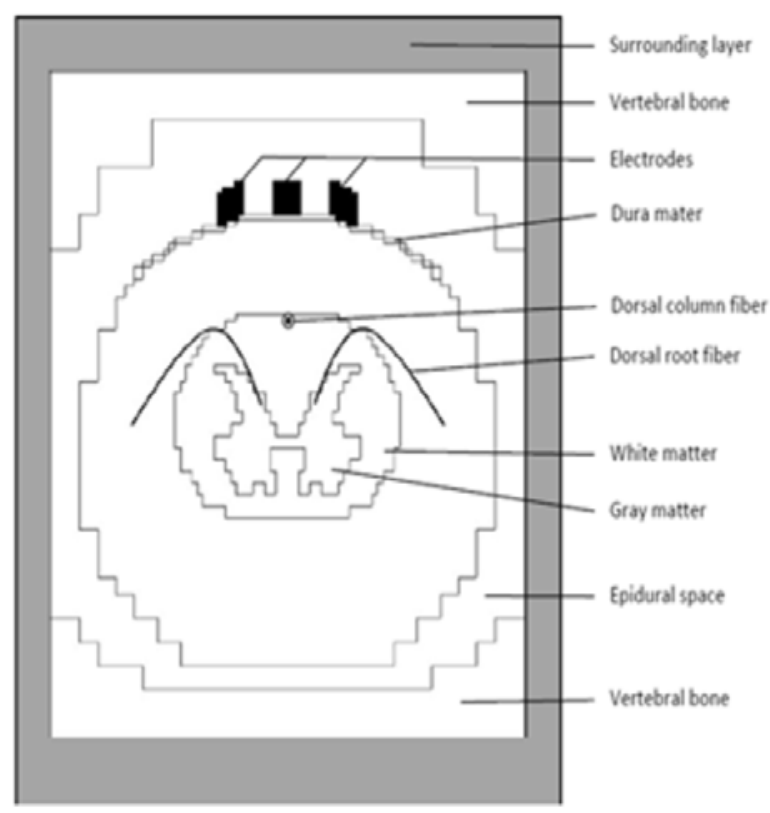

Figure 1 Transverse cross-section of the low-thoracic spinal cord stimulation model. The anatomical compartments and the epidural lead are labeled.

\subsubsection{Lead models}

In order to evaluate the influence of Anode Intensification (AI), lead types with different configurations and contact spacing were modeled.

Aligned Transverse Tripolar configuration using triple percutaneous leads. This percutaneous triple lead configuration consists of three contacts in the transverse plane, namely a central cathode and two lateral anodes. The cathode and anodes are at the same rostro-caudal level (aligned). The gap or medio-lateral spacing between the adjacent leads is $1.5 \mathrm{~mm}$ edge-to-edge. The resulting medio-lateral span, defined as the center-to-center (C-C) distance of the outer electrodes, is $5.0 \mathrm{~mm}$. The configuration is abbreviated PERC AL.

Longitudinal Guarded Cathode (LGC) using single percutaneous lead. This percutaneous single lead configuration consists of three contacts parallel to the longitudinal spinal axis. Longitudinal tripolar (+-+) contact combination was modelled on the lead. The center-to-center (C-C) contact spacing is $4.0 \mathrm{~mm}$. this is the commercially available contact spacing. 
Longitudinal Guarded Cathode with wide spacing (LGC+) using single percutaneous lead. This percutaneous single lead configuration consists of three contacts parallel to the longitudinal spinal axis. Longitudinal tripolar (+-+) contact combination was modeled on the lead. The center-to-center (C-C) contact spacing is $4.5 \mathrm{~mm}$.

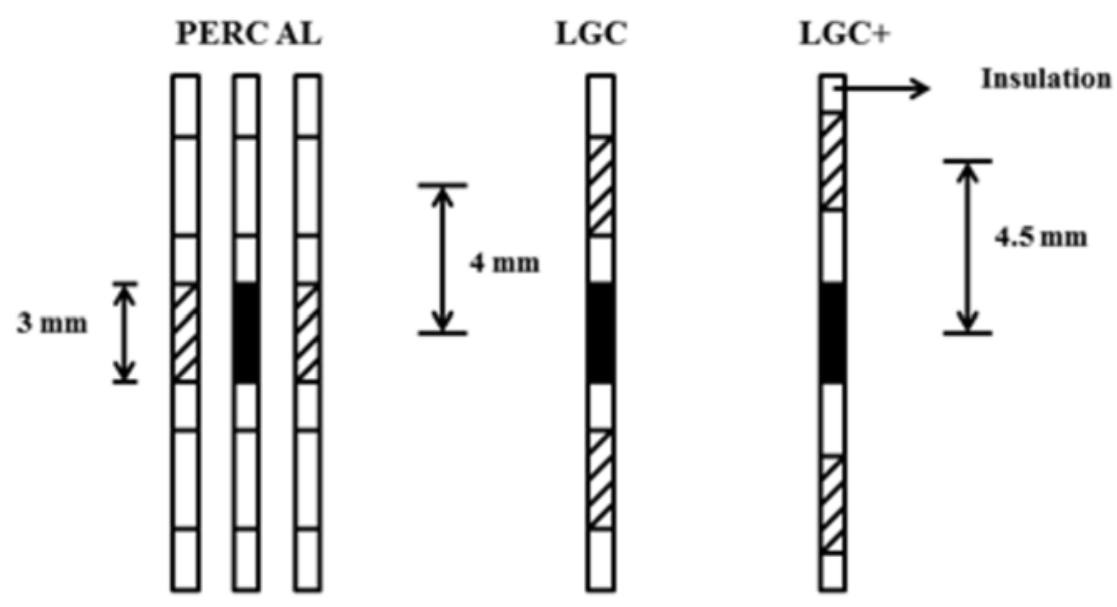

Figure $22 D$ projections of the lead models. PERC AL-Aligned transverse tripoles using triple percutaneous leads. LGC using a single percutaneous lead. The C-C contact spacing is $4.0 \mathrm{~mm}$. LGC+using single percutaneous lead. The C-C contact spacing is $4.5 \mathrm{~mm}$. The small size of the spacing step was modelled to study the local effects around the commercially available contact spacing of 4.0mm. The anodes are hatched and the cathodes are filled in all the three lead configurations.

All three lead types have contacts of the same shape and size. The length of the contacts is $3 \mathrm{~mm}$ and the width and height of the contacts are $1 \mathrm{~mm}$ each. In all the lead configurations, the lead position was always symmetrical with respect to the spinal cord midline. The spinal cord midline in our model coincides with the radiological midline. The leads were placed just behind the dura mater, which is also the most favourable position in clinical applications.

\subsubsection{Nerve fiber model}

In order to quantify the effect of Anode Intensification (AI), the response of both DC and DR fibers was simulated. The DC fiber model represented a $12 \mu \mathrm{m}$ diameter, straight, myelinated fiber whereas the DR fiber model represented a $15 \mu \mathrm{m}$ diameter, curved, myelinated fiber. The kinetics of the fiber membrane as described by Wesselink et al. was used (15). The position of the DC fiber model was varied in order to determine the extent of the fiber recruitment in the DCs. Similarly, the longitudinal span of DR recruitment was determined by varying the rostro-caudal position of the DR fiber model. The DC and DR 
fiber position was varied within their anatomical constraints. For details on the fiber models see Sankarasubramanian et al. 2011 (20).

\subsubsection{Stimulation strategy}

Stimulation by current-controlled pulse generators giving simultaneous pulses of the same pulse width but independent current control at each contact was modelled. In this way the current applied to each contact could be specified. Both current balance and Anode Intensification (AI) conditions were modelled on the LGC, LGC+ and PERC AL configurations. The anodal currents were increased by $10 \%, 20 \%$ and $30 \%$, while the cathodal currents were kept constant.

\subsubsection{Output parameters}

To enable a quantitative comparison of the performance of the modeled percutaneous and surgical lead types, several model output parameters were calculated. These parameters are defined below.

- $\mathrm{I}_{\mathrm{DC}}(\mathrm{mA})$ : DC fiber threshold, corresponding to the lowest activation threshold among all DC fibers.

- $\quad \mathrm{I}_{\mathrm{DR}}(\mathrm{mA})$ : DR fiber threshold, corresponding to the lowest activation threshold of the DR fiber. It is calculated as the minimum of left DR fiber threshold $\left(\mathrm{I}_{\mathrm{DR}, \mathrm{L}}\right)$ and right $\mathrm{DR}$ fiber threshold $\left(\mathrm{I}_{\mathrm{DR}}\right.$, R).

- $\mathrm{I}_{\mathrm{PT}}(\mathrm{mA})$ : Paresthesia threshold, corresponding to the current required to activate the lowest threshold fiber, being either a DC or DR fiber. Hence, it is calculated as the lowest value between $\mathrm{I}_{\mathrm{DC}}$ and $\mathrm{I}_{\mathrm{DR}}$.

- $\mathrm{I}_{\mathrm{DT}}(\mathrm{mA})$ : Discomfort threshold, corresponding to the current at initial stimulation of proprioceptive DR fibers. It is defined as $\mathrm{I}_{\mathrm{DT}}=1.4 * \mathrm{I}_{\mathrm{DR}}(21)$.

- UR: Usage range, defined as the ratio between $\mathrm{I}_{\mathrm{DT}}$ and $\mathrm{I}_{\mathrm{PT}}$. $\mathrm{UR}=\mathrm{I}_{\mathrm{DT}} / \mathrm{I}_{\mathrm{PT}}$.

- $\mathrm{S}_{\mathrm{RA}}\left(\mathrm{mm}^{2}\right)$ : Maximum recruited DC area, which is the area between the dorsal border of the DCs and the recruitment contour at $\mathrm{I}_{\mathrm{DT}}$.

- $\mathrm{Z}_{\mathrm{TIS}}(\mathrm{Ohm})$ : Tissue impedance, between the anodes and the cathodes. $\mathrm{Z}_{\mathrm{TIS}}=\mathrm{Z}_{\text {Cathode }}+0.5 \mathrm{Z}_{\text {Anode }}$ for a transverse tripolar configuration. $\mathrm{Z}_{\text {Cathode }}$ and $\mathrm{Z}_{\text {Anode }}$ are the impedances at the cathode and the anode respectively.

- $\mathrm{Z}_{\mathrm{W}}(\mathrm{Ohm})$ : Wire impedance between the lead contact and the stimulator output.

- $\mathrm{Z}_{\mathrm{G}}(\mathrm{Ohm})$ : Load or Total impedance, seen between the stimulator outputs. $\mathrm{Z}_{\mathrm{G}}=\mathrm{Z}_{\mathrm{TIS}}+1.5 \mathrm{Z}_{\mathrm{W}}$ for a tripolar configuration. 
- $\quad \mathrm{E}_{\mathrm{DT}}(\mu \mathrm{J})$ : Energy per pulse at $\mathrm{I}_{\mathrm{DT}}$ delivered by the stimulator. It is calculated as $\mathrm{E}_{\mathrm{DT}}=\mathrm{I}_{\mathrm{DT}}{ }^{2} * \mathrm{Z}_{\mathrm{G}}$ * $\mathrm{T}$ (Equation 1), where $\mathrm{T}$ is the pulse width, being $210 \mu \mathrm{sec}$ in all simulations.

Transverse tripolar stimulation and longitudinal tripolar stimulation using the configurations enabled the estimation of $I_{D C}$ and $I_{D R}$ at various anodal current ratios. The area of recruited fibers in the DCs as well as the energy consumption at $\mathrm{I}_{\mathrm{DT}}$ was also calculated.

\subsection{Results}

\subsubsection{Transverse tripoles with anode intensification}

TTS was performed using PERC AL for both current balance, and a 10\% Anode Intensified (AI) situation. The $\mathrm{I}_{\mathrm{DC}}$ and $\mathrm{I}_{\mathrm{DR}}$ were calculated and the respective DC recruitment contours were plotted at $\mathrm{I}_{\mathrm{DT}}$ (figure 3). It can be seen from figure 3 (b) that TTS with 10\% AI recruits a smaller medial DC recruited area as compared to the current balance situation at $\mathrm{I}_{\mathrm{DT}}$ shown in figure 3 (a). Both, a smaller depth and width of medial DC area is activated at $\mathrm{I}_{\mathrm{DT}}$. Also, as depicted in figure 3 (c), TTS at current balance at $40 \% \mathrm{I}_{\mathrm{DT}}$ recruited a DC area similar to the one achieved with $10 \% \mathrm{AI}$ at $\mathrm{I}_{\mathrm{DT}}$.

Therefore, AI of TT clearly has an effect on the area of recruitment of DC fibers. However, since the same effect can be achieved at lower stimulation currents in a current balance situation, AI of TTS is definitely not advantageous.

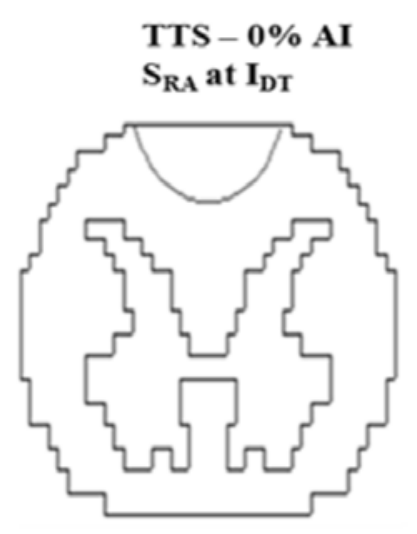

(a)

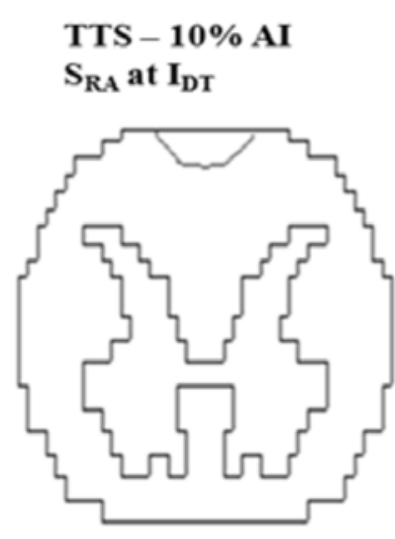

(b)

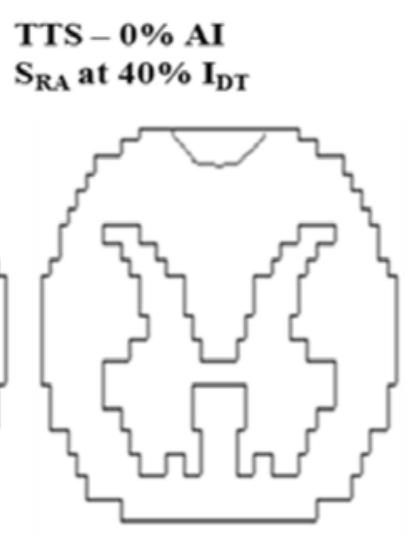

(c)

Figure 3 (a) TTS using PERC AL with 0\% AI (current balance). The maximum DC recruited area $\left(S_{R A}\right)$ at $I_{D T}$ is shown. (b)Transverse tripolar stimulation using PERC AL with $10 \%$ AI. The $S_{R A}$ at $I_{D T}$ is shown. (c) DC recruitment contours for TTS using PERC AL at current balance, at a stimulation current of $40 \%$ $I_{D T}$. 


\subsubsection{Longitudinal tripoles with anode intensification}

The effects of tripolar stimulation using LGC and LGC+ were analysed at current balance, and a 10\%, $20 \%$ and $30 \%$ AI situation. The calculated values of $I_{D T}$, UR, $E_{D T}$ and $S_{R A}$ are shown in table 1 for the respective configurations.

Tripolar stimulation of LGC with $10 \%, 20 \%$ and $30 \%$ AI recruits a $16 \%$, 33\% and $58 \%$ larger $\mathrm{S}_{\mathrm{RA}}$ than a balanced current situation. Similarly, tripolar stimulation of LGC+ with 10\%, 20\% and 30\% AI recruits a $16 \%, 27 \%$ and $45 \%$ larger $S_{\mathrm{RA}}$ than a balanced current situation. Therefore, AI of both LGC and LGC+ has a significant effect on the DC recruitment areas. The AI effect on the DC recruitment areas of LGC is higher as compared to the LGC+.

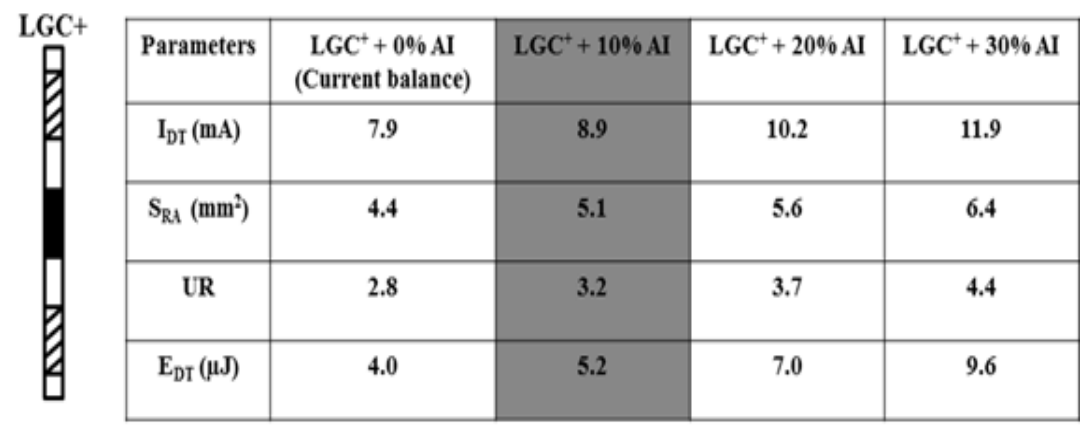

\begin{tabular}{|c|c|c|c|c|}
\hline Parameters & $\begin{array}{c}\text { LGC }+0 \% \text { AI } \\
\text { (Current balance) }\end{array}$ & LGC + 10\% AI & LGC + 20\% AI & LGC + 30\% AI \\
\hline $\mathrm{I}_{\mathrm{DI}}(\mathrm{mA})$ & 9.6 & 11.4 & 13.9 & 17.9 \\
\hline $\mathrm{S}_{\mathrm{RA}}\left(\mathrm{mm}^{2}\right)$ & 4.5 & 5.2 & 6.0 & 7.1 \\
\hline $\mathrm{UR}$ & 3.1 & 3.6 & 4.4 & 5.7 \\
\hline $\mathrm{E}_{\mathrm{DI}}(\mu \mathrm{J})$ & 5.7 & 8.2 & 12.5 & 20.8 \\
\hline
\end{tabular}

Table 1 Calculated $I_{D T}$, maximum DC recruited areas $\left(S_{R A}\right)$, usage range (UR) and energy consumptions $\left(E_{D T}\right)$ for tripolar stimulation of LGC and LGC+ with $0 \%, 10 \%, 20 \%$ and $30 \%$ anode intensification (AI). The shaded columns represent the comparison of LGC+ with $10 \%$ AI to LGC without AI described in the text. 


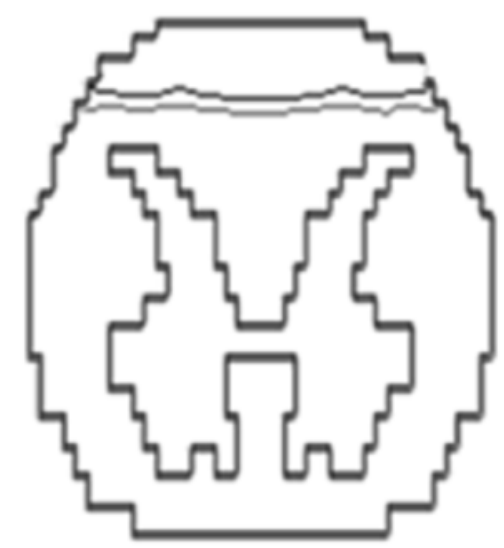

Figure 4 Recruitment contours in the DCs for tripolar stimulation of LGC+ with $10 \%$ AI (depicted by the grey line) and LGCs without AI (depicted by the black line).

Similar to $\mathrm{S}_{\mathrm{RA}}$, the UR is $14 \%, 32 \%$ and $57 \%$ larger in tripolar stimulation of LGC+ with $10 \%, 20 \%$ and $30 \%$ AI. The UR undergoes a larger change when AI is performed on the LGC. The increase in UR is $16 \%, 42 \%$ and $84 \%$ respectively.

The above results clearly predict that AI of both LGC and LGC+ can provide a larger $\mathrm{S}_{\mathrm{RA}}$ and UR compared to a balanced current situation. A larger $S_{R A}$ and $U R$ is advantageous in eliciting broader paresthesia coverage.

Direct comparison of the DC recruitment contours of LGC without AI and LGC+ with $10 \%$ AI is shown in figure 4. It can be seen that a $10 \% \mathrm{AI}$ of $\mathrm{LGC}+$ recruits a larger $\mathrm{S}_{\mathrm{RA}}$. It can also be seen from table 1 that a $10 \% \mathrm{AI}$ of LGC+ recruits a larger $\mathrm{S}_{\mathrm{RA}}$ and $\mathrm{UR}$ at lower $\mathrm{E}_{\mathrm{DT}}$ than that of stimulation of LGC without AI. Hence, AI of LGC+ is more efficient than the performance of LGC with conventional stimulation techniques. To a larger benefit, this can be attained at lower energy consumption.

\subsection{Discussion}

The primary aim of this computer modelling study was to investigate if enhanced DC recruitment is achieved when anodal currents in transverse tripolar and longitudinal tripolar (LGC and LGC+) contact combinations are increased up to $30 \%$ with respect to the cathodal current. Secondly, the merits of anodal intensification (AI) are evaluated by comparing the DC recruitment areas and energy consumption of LGC+ with AI, against stimulation using a LGC without AI. 


\subsubsection{Transverse tripoles with anode intensification}

From section 3.1 of the results, it is evident that AI of TTS clearly has an effect on the area of recruitment of DC fibers. Previous mathematical modeling has shown that TTS using three aligned percutaneous leads (PERC AL) can recruit a region of activation that is focussed to the medial DC fibers (22). As a potential improvement to recruit deeper DC fibers, AI of TTS using PERC AL was performed in this study. The electric fields from the stimulation and the resulting nerve fiber recruitment were calculated to see if the depth of penetration of medial DC fibers can be increased, without lateral spread of activation. It was observed that the $10 \%$ intensified TTS activated a smaller width of the medial DCs, as compared to the balanced current stimulation. This is because the intensified anodal currents seem to have hyperpolarised the lateral DCs, thus rendering them more resistant to depolarization. The net effect of the hyperpolarisation pushed the area of activation away from the anode, thus shaping the stimulated area of DC activation. The result is a recruitment of smaller width and depth of medial DCs.

Intensifying the anodes further (more than 10\%) resulted in extremely high perception threshold currents $\left(\mathrm{I}_{\mathrm{PT}}\right)$, with an absence of DC recruited area. It is worth recalling here that the medio-laterally aligned electrodes in TTS produce a stimulation field that is perpendicular to the orientation of the DCs of the spinal cord (7). Such a stimulation field requires high amplitudes of currents to activate the DCs and therefore produce better penetration of the cord. In a balanced current configuration, these high currents were sufficient to activate the large fibers of the DCs, resulting in deep penetration of the medial DCs. However, intensification of anodes by more than $10 \%$ produced extremely high threshold currents that could not stimulate deeper fibers of the medial DCs.

Although AI of TTS has an effect on the area of recruitment of DC fibers, it was at the expense of large stimulation currents. A similar depth and width of medial DC recruitment was observed when TTS was performed on the PERC AL without AI. At stimulation currents $40 \%$ lesser than $\mathrm{I}_{\mathrm{DT}}$, such recruitment was possible. Therefore, AI of transverse tripoles is definitely not advantageous in obtaining enhanced recruitment of DCs.

\subsubsection{Longitudinal tripoles with anode intensification}

Our aim was to investigate if less DR stimulation and enhanced DC stimulation was observed, when anodal currents in LGC and LGC+ are increased up to 30\% with respect to the cathodal current. From section 3.2 of the results, it is evident that AI of both LGC and LGC+ can provide a larger $\mathrm{S}_{\mathrm{RA}}$ and UR compared to a balanced current situation. A larger $S_{R A}$ and $U R$ is advantageous in eliciting broader paresthesia coverage. 
Compared to LGC+ stimulation without $\mathrm{AI}$, we found a significant increase in $\mathrm{I}_{\mathrm{DR}}$ and hence the $\mathrm{I}_{\mathrm{DT}}$ under the intensified LGC+ configuration with up to 30\% AI. The increase in DR thresholds implies that DRs are more sensitive to the direction of current flow and are therefore difficult to stimulate. $\mathrm{I}_{\mathrm{DC}}$ which determined the $\mathrm{I}_{\mathrm{PT}}$, did not change much in comparison with $\mathrm{I}_{\mathrm{DT}}$. The usage range, UR, defined as the ratio of $\mathrm{I}_{\mathrm{DT}}$ and $\mathrm{I}_{\mathrm{PT}}$ was strongly influenced by $\mathrm{I}_{\mathrm{DT}}$. UR is an indicator of the extent of DC activation and thus the extent of maximum paresthesia coverage. Large UR permits increased activation of DC fibers. A greater depth of DC recruitment is obtained as compared to the stimulation of LGC+ without AI.

Similar to the performance of LGC+, LGC with up to $30 \%$ AI also recruited a larger UR and $\mathrm{S}_{\mathrm{RA}}$ compared to that of LGC without AI. In the LGC, the cathode is flanked by 2 anodes that are more closely spaced than in LGC+. The result is an improved characteristic of the super- positioned cathodic and anodic stimulation fields. Based on earlier simulations of activation functions (AF) by Holsheimer (23), it can be recollected that an increased positive cathodic AF manifests in an increased depolarization and a decreased stimulation threshold of DC fibers $\left(\mathrm{I}_{\mathrm{DC}}\right)$. The DR thresholds $\left(\mathrm{I}_{\mathrm{DR}}\right)$ are high owing to the intensified anodal currents. Therefore, the perception threshold $\left(\mathrm{I}_{\mathrm{PT}}\right)$ is lower and the discomfort threshold $\left(\mathrm{I}_{\mathrm{DT}}\right)$ is higher, resulting in a larger UR and paresthesia area as compared to LGC without AI.

The concept of longitudinal tripoles differs basically from TTS in that the longitudinal guarding effect of the tripoles is more predominant than the lateral anodal shielding effect of the anodes. In TTS with three aligned percutaneous leads (PERC AL), the lateral leads that are placed on the left and right of the midline, closer to the DRs, are the ones that tend to effect DR stimulation. Leads placed on the midline, as in LGC and LGC+, and effecting longitudinal tripolar stimulation show balanced left-right stimulation and tend to produce less DR stimulation. Moreover, least stimulation current is needed for DC fiber activation in longitudinal tripolar stimulation, where the main direction of the current is parallel to the axes of the nerve fibers.

A large UR and improved DC recruitment is accompanied by a substantial increase in energy consumption, particularly with AI of LGC. This is due to the greater proximity of anodes and cathodes as compared to that of LGC+, resulting in a more confined electric field. The effect of AI can be better interpreted when comparing the $\mathrm{S}_{\mathrm{RA}}$, UR and $\mathrm{E}_{\mathrm{DT}}$ of LGC+ with AI to that of LGC without AI. It was observed that a $10 \% \mathrm{AI}$ of LGC+ recruited a larger $\mathrm{S}_{\mathrm{RA}}$ and $\mathrm{UR}$ at lower $\mathrm{E}_{\mathrm{DT}}$ than stimulation of LGC without AI. Hence, an improved performance of LGC+ can be achieved with AI as compared to LGC without AI. The larger $\mathrm{S}_{\mathrm{RA}}$ and UR of the anode intensified LGC+ at lower $\mathrm{E}_{\mathrm{DT}}$ as compared to the nonintensified LGC are significant factors for the efficacy of the AI technique. Therefore, AI of LGC+ may be more efficient than the performance of LGC with conventional stimulation techniques. However, it 
should be noted that increase in $\mathrm{S}_{\mathrm{RA}}$ and UR with AI and also at higher percentages of AI is important in itself, although it may be at the expense of higher energy consumption and narrow inter-electrode spacing.

Contact spacing's larger than $4.5 \mathrm{~mm}$ were not considered in this study. Further increase of the contact spacing will eventually result in monopolar stimulation in which the anodal currents no longer contribute to the sharpening of the combined electric field (required for increasing the activation function). Of course AI will increase the strength of the anodal contributions to the combined electric field also at larger spacing's, but not the sharpening of this field, as the curvature of the anodal field's decreases with distance. Although it would be interesting to explore the interaction between the effect of AI and increased contact spacing beyond the transition from bipolar to monopolar stimulation, these simulations were outside the scope of this study.

\subsubsection{Limitations of the model}

In this modeling study, we have not investigated whether electric fields created from different types of stimulation configurations (longitudinal versus transverse tripole) may activate smaller and larger diameter fibers differently, resulting in different patterns of stimulated DC fibers. It is assumed that $\mathrm{I}_{\mathrm{PT}}$ is immediately related to the activation of those first cutaneous fibers (either DC or DR fibers) having the lowest threshold. Since large nerve fibers have lower stimulation thresholds than small nerve fibers, the large nerve fibers will normally be stimulated before small nerve fibers, when located at the same distance from the active electrode. Therefore, the authors simulated only the largest cutaneous afferent fiber parts (either DC or DR fibers). Because of this, recruitment of smaller fibers is not modelled. We however hypothesize that AI of LGC and LGC+ can preferentially recruit smaller diameter fibers in the DC and enable more efficient recruitment of total DC fibers than the TTS configuration.

\subsection{Conclusions}

This study provides insight of significant correlation between AI of longitudinal and transverse tripoles, and DC paresthesia. The results strongly support the use of longitudinal tripolar stimulation as a means of improving the DC paresthesia in SCS. LGC+ and LGC with AI allow additional DC stimulation, which may increase the likelihood of activating fibers inaccessible with conventional programming. Also, LGC+ with AI can be more efficient than LGC without AI, as a larger DC recruited area and UR is achieved at lower energy expenditure. AI of transverse tripoles is not advantageous, as the same DC recruitment can be achieved at lower stimulation amplitudes with balanced anodal and cathodal currents. 


\section{Acknowledgements}

The authors gratefully thank Boston Scientific Neuromodulation (Valencia, CA, USA) for their grant to support this research.

\section{References}

[1] Hegarty D. Spinal cord stimulation: The clinical application of new technology (Review article). Anesthesiology Research and Practice. 2011.

[2] Alo KM, Redko V, Chamov J. Four year follow-up of dual electrode spinal cord stimulation for chronic pain. Neuromodulation. 2002;5:79-88.

[3] Barolat G. Current status of epidural spinal cord stimulation. Neurosurgery. 1995;5:98-124.

[4] North RB, Ewend MG, Lawton MT, Piantadosi S. Spinal cord stimulation for chronic, intractable pain: superiority of 'multi-channel' devices. Pain. 1991;44:119-130.

[5] Simpson BA. Spinal cord stimulation. Pain Reviews. 1994;1:199-230.

[6] Oakley JC, Prager JP. Spinal cord stimulation: Mechanisms of action. Spine. 2002;27:2574-2583.

[7] Struijk JJ, Holsheimer J. Transverse tripolar spinal cord stimulation: theoretical performance of a dual channel system. Med. Biol. Eng. Comput.1996;34:273-279.

[8] Holsheimer J, Nuttin B, King GW, Wesselink WA, Gybels JM, de Slutter P. Clinical evaluation of paresthesia steering with a new system for spinal cord stimulation. Neurosurgery.1998;42:541-549.

[9] Oakley JC. Spinal cord stimulation in axial low back pain: solving the dilemma. Pain Medicine 7. 2006;S58-63.

[10] Wesselink WA, Holsheimer J, Boom HBK. Analysis of current density and related parameters in spinal cord stimulation. IEEE Transactions on Rehabilitation Engineering. 1998;6:200-207.

[11] Holsheimer J. Effectiveness of spinal cord stimulation in the management of chronic pain: analysis of technical drawbacks and solutions. Neurosurgery. 1997;40:990-999.

[12] Holsheimer J, Wesselink WA. Effect of anode-cathode configuration on paresthesia coverage in spinal cord stimulation. Neurosurgery. 1997;41:654-659.

[13] Manola L, Holsheimer J, Veltink P. Technical performance of percutaneous leads spinal cord stimulation: a modeling study. Neuromodulation. 2005;8:88-99.

[14] Holsheimer J, Struijk JJ, Tas NR. Effects of electrode geometry and combination on nerve fiber selectivity in spinal cord stimulation. Med Biol Eng Comput. 1995;33:676-682.

[15] Wesselink WA, Holsheimer J, King GW, Torgerson NA, Boom HBK. Quantitative aspects of the clinical performance of transverse tripolar spinal cord stimulation. Neuromodulation. 1999; 2:5-14.

[16] Holsheimer J. Computer modeling of spinal cord stimulation and its contribution to therapeutic efficacy (review). Spinal Cord. 1998;36:531-540. 
[17] Struijk JJ, Holsheimer J, Boom HBK. Excitation of dorsal root fibers in spinal cord stimulation: a theoretical study. IEEE Trans. Biomed. Eng. 1993;40:632-639.

[18] Holsheimer J, Den Boer JA, Struijk JJ, Rozeboom AR. MR assessment of the normal position of the spinal cord in the spinal canal. Am J Neuroradiol. 1994;15:951-959.

[19] Manola L, Holsheimer J. Technical performance of percutaneous and laminectomy leads analyzed by modeling. Neuromodulation. 2004;7:231-241.

[20] Sankarasubramanian V, Buitenweg JR, Holsheimer J, Veltink P. Triple leads programmed to function as longitudinal guarded cathodes in spinal cord stimulation: a modeling study. Neuromodulation. 2011;14:401-411.

[21] He J, Barolat G, Ketcik B. Stimulation usage range for chronic pain management. Analgesia. 1995;1: 75-80.

[22] Sankarasubramanian V, Buitenweg JR, Holsheimer J, Veltink P. Electrode alignment of transverse tripoles using a percutaneous triple-lead approach in spinal cord stimulation. J. Neural Eng. 2011. doi:10.1088/1741-2560/8/1/016010.

[23] Holsheimer J. Principles of neurostimulation. Electrical stimulation and the relief of pain. Pain research and clinical management. 2003;15:17-36. 
Chapter 5 


\section{CHAPTER 6}

General discussion and final remarks 
Chapter 6 
In this thesis, the clinical and technical aspects of stimulation optimization techniques for chronic pain relief in SCS are presented. The optimization techniques are aimed to focus primarily on improving SCS equipment. In particular, the performance of novel percutaneous and surgical triple-lead configurations, with both longitudinal and transverse tripolar contact combinations, is investigated in a current-controlled stimulation approach.

\subsection{Improving SCS equipment}

Improving the equipment can result in increasing the efficacy of SCS. This is possible, if the improvement increases the number of successful outcomes in SCS, for example, by making the implantation technique fail-safe. The success of SCS also depends on the ability of the physician to choose the best available equipment to treat a specific pain condition. The technical goal of SCS in chronic neuropathic pain is to cover, or mask, the patients' pain area with a stimulation-induced paresthesia; this pain/paresthesia overlap is a necessary, but not sufficient condition to achieve pain relief (1). A further technical goal is to avoid the perception of extraneous stimulation. Lead design, and lead placement/alignment, together with IPG design are vital in achieving the above mentioned goals. Clinically relevant paresthesia, which exists in a limited usage range between perception and discomfort, can be enhanced by optimizing the above mentioned factors. Studies performed with the UT-SCS computer model provide a theoretical basis for decisions about the design, and placement of leads and IPGs and about ways to eventually improve SCS equipment.

\subsubsection{Lead design}

In SCS, theoretical models have helped clinicians better understand potential differences in current density, neuron activation patterns, and fiber selectivity. This has led to the design of a new generation of multi-column contact arrays that allow not only the use of longitudinal stimulation fields but also the activation of transverse stimulation fields. Some of the core design options for SCS leads include the number of active contact arrays, assignment of appropriate contact combinations and both longitudinal and transverse contact array spacing.

Number of electrode contact arrays: Earlier clinical studies and modeling work have shown that the parallel orientation and medial location of nerve fibers in the DCs favour the use of single and dual percutaneous contact arrays parallel to the axis of the spinal cord (2). In SCS therapy, clinical experience widely varies and the opinions still differ as to the usefulness of single and dual-lead stimulation approaches. The reality of SCS clinical practice, however, indicates that most physicians prefer using more than one percutaneous contact array. Many physicians have noted that the implantation of dual 
percutaneous lead arrays with 4 or 8 contacts has increased success rates among them who have technical difficulty capturing the ideal stimulation target area with a single contact array (3). However, chronic pain which is often present in diffuse distributions can have secondary pain areas that cannot be treated even with a dual-lead system. Treating wide areas of pain often poses quite a challenge. Considering the above challenges and observations, triple-, both percutaneous and surgical leads were modeled with the aim to provide a theoretical explanation and justification of the expected clinical phenomena (Chapter 2, chapter 3 , and chapter 4).

Appropriate electrode contact combinations: Effectiveness of SCS therapy can be improved by choosing appropriate electrode contact combinations to maximize paresthesia coverage (4). Maximizing paresthesia coverage maximizes the therapeutic effect of SCS. Multiple-lead arrays configured as longitudinal guarded cathodes (+-+), LGCs, definitely seem to improve therapeutic capabilities by directing the stimulation field within the DCs in parallel to the DC fibers to obtain reliable and reproducible paresthesia covering the pain area (5). Moreover, such a configuration is shown to be chosen by patients significantly more than other contact configurations (6). Transverse guarded cathode or transverse tripolar configurations, on the other hand, possess an added ability to produce independently-controlled currents from each anode (This added ability depends on the ability of the stimulator-only dual source stimulator can achieve this). This is capable of steering the electrical field deeper and also from right to left across the DCs (7). It also permits high-amplitude stimulation without the negative sensory and motor effects as observed with single or dual quadripolar leads. We demonstrated that LGCs (+-+) configured on triplelead percutaneous arrays, can serve as a potential improvement to dual-lead percutaneous arrays in covering a larger width of the low-thoracic DCs (Chapter 2). Triple percutaneous leads are shown to be desirable because of their ability in coping with medio-lateral displacement of the single and dual percutaneous leads, which is a common clinically-observed phenomenon. By doing so, triple-lead percutaneous arrays are predicted to help achieve a greater post-operative flexibility than single and dual leads in modifying the stimulation-induced electric field. This can in turn help enable activation of multiple dermatomes in the patient. Widespread and complex pain complaints can therefore best be addressed with such a triple-lead percutaneous array configuration. There are also likely risks involved in inserting multiple percutaneous leads into the epidural spinal cord layer of the patient. (1) Inappropriate patient anatomies and improper lead anchoring during implantation can attribute to increase in lead fracture and lead migration rates respectively (8). This can in turn result in technical failures such as loss of paresthesia coverage and repeated lead revisions. (2) Appropriate transversal spacing between the implanted percutaneous leads can be difficult to achieve and maintain. However, simple measures, such as, proper attention to selection of patient anatomies together with the adoption of novel, clinically 
emerging methods of securing percutaneous lead arrays can ameliorate the above mentioned technical errors. An important aspect to percutaneous triple lead implantation is that it always provides the physician an option and hence a potential advantage of any two leads being chosen for stimulation. The choice can be made based on the pain condition of the patient. Currently available IPGs are limited in the number of stimulating contacts that can be accommodated. Triple percutaneous leads, if technically and clinically successful, on the long-run can help improve the equipment for SCS therapy and encourage device manufacturers to create more flexible and versatile stimulation devices.

Transverse contact spacing: It was also shown from our computer modeling study that the transverse spacing between the inserted percutaneous lead arrays is a major determinant of the area and distribution of paresthesia (Chapter 2). The result breaks one of the basic postulates of current SCS implantation practice - it clearly predicts that putting two leads closer to each other will improve the electrical penetration of DCs and increase the usage range of stimulation. Clinical experience suggests that SCS systems which allow for current steering between the lead contacts should have the leads placed not more than $4 \mathrm{~mm}$ apart so that current can be directed to the midline. A rule of thumb is that if the CSF layer is thin (indicated by relatively low midline perception thresholds), then the lead arrays may be placed closer together. Conversely, a thick CSF layer would suggest a wider lead separation. Our modeling study has indicated that, apart from the CSF thickness factor, placing the lead arrays closer together is vital in obtaining broad paresthesia areas in the patient. Broad paresthesia areas are likely to be achieved at the expense of large power consumption, due to the close proximity of the implanted lead arrays. Definitely, a trade-off between therapeutic effect and power saving has to be made. Increased power consumption may be acceptable if it brings benefits for the patient, especially as the current generation of compact rechargeable IPGs meets the power requirements of SCS.

\subsubsection{Lead placement and alignment}

One can also optimize effectiveness of SCS by minimizing the incidence of complications. From a clinical perspective, meticulous attention to lead placement, lead alignment, and implantation technique can help reduce complications; for example, new methods of securing percutaneous lead arrays can eliminate longitudinal migration (9). In addition, understanding the lead design (both percutaneous and surgical) and using intelligent electrode contact combinations can help restore lost paresthesia coverage. Recently, transverse tripoles using three parallel percutaneous leads were used effectively in the clinic to treat low-back pain (10). The leads were placed in the dorsal epidural space, with the electrodes in the two lateral leads functioning as anodes and the electrode in the midline lead as a cathode. Sooner or later, loss of therapeutic effect can occur in the patient, if lead offset takes place in the longitudinal direction due to 
various reasons (lead migration, frequent postural changes etc). We explored this clinically-relevant problem by modeling the influence of electrode alignment of transversely oriented guarded cathodes on the paresthesia coverage of pain area (Chapter 3). We showed that selective targeting of either medial or lateral DC fibers is lost, if the alignment of the transverse tripoles is not achieved or lost later in time (Chapter 3). Our subsequent study revealed that this loss can be repaired by stimulation using the adjacent anodal contacts on the lateral percutaneous leads of the transverse tripolar configuration (Chapter 4). Moreover, it was predicted that the same quadripolar anodal configuration on a three-column surgical lead can provide an improved performance in treating low-back pain, as compared to its percutaneous replica (Chapter 4). With a three-column, 16-contact surgical lead already available in the market, recent technological advances offer significant clinical perspectives for new therapeutic applications of transverse tripolar stimulation paradigms, in particular to address the low- back pain component. However, the current literature does not provide sufficient data on the use or clinical evaluation of this three-column surgical lead. Also, it was predicted by our study, that such a lead having widely-spaced contacts in the longitudinal direction cannot selectively stimulate the DCs and is therefore not the best configuration to treat low-back pain (Chapter 4).

\subsubsection{Choice of leads}

Very few studies have directly compared surgical versus percutaneous lead implantation. Those that did reveal that surgical leads exhibit lesser long-term migration rates and better long-term survival rates when compared to percutaneous leads $(11,12)$. Surgical leads also possess an added advantage of being placed directly on the dura under visual guidance. This is likely an argument in favour of their choice, particularly at implantations performed in the low-thoracic vertebral region. This together with the fixed transversal lead spacing (spacing between the columns of electrode contacts is fixed) factor, support the general trend towards the growing clinical usage of surgical leads with more columns of closely-spaced electrode contacts. With advancements in technology, surgical leads have also become thinner and more pliable. Moreover, the improved steer ability of the leads combined with the recent design of the Epiducer lead delivery system (St. Jude Medical) has allowed the advancement of surgical leads even without the use of a laminectomy. Due to these advantages, some centers prefer to use surgical leads as their first choice. The development of the 5-column surgical lead from Penta, St. Jude Medical, approved by the FDA in 2009, has been shown to provide improved programmable capabilities and possible treatment outcomes. In a recent study on 5 patients implanted with the lead (13), it was observed that the patients reported excellent paresthesia coverage. First clinical experiences with the 5-column surgical lead from Penta, St Jude Medical, support the general hypothesis that fine control of lateral current distribution of stimulation is effective in providing a broad area of coverage. The capacity for precise targeting and 
focussed stimulation of low-back fibers is also possible. Low-back and leg capture was obtained with lateral steering of current across the 5-column array. However, long term follow-up is vital and larger study groups are necessary to obtain valid data.

\subsubsection{IPG design}

Some of the important design options for an IPG include its power source (current or voltage source), how it is connected to the electrode, and number of independent channels. Chapter 5 provided insight of significant correlation between the technique of anode intensification (AI) of longitudinal and transverse tripolar configurations, and DC paresthesia. Our computational modeling results predicted that, longitudinal guarded cathodes (LGC) and LGC+ with AI allow for additional DC stimulation and reduced DR stimulation, which may increase the likelihood of activating fibers inaccessible with conventional programming techniques. Importantly, the AI technique requires a current instead of a voltage source, and independent control of the currents to the respective electrodes. Such a unique feature is currently available only in Precision IPGs systems from Boston Scientific Corporation. Theoretical and clinical work to validate the concept of AI technique is necessary and is yet to be performed. AI of transverse tripoles is found not to be advantageous over AI of longitudinal tripoles, as the same DC recruitment is achieved at lower stimulation amplitudes with balanced anodal and cathodal currents (Chapter 5).

\subsection{Validity of the model and future outlook}

Computer modeling provides a considerable contribution in the knowledge of physiological effects of SCS. Improved scientific understanding from computer modeling will allow for more efficacious application of neurostimulation technology to patients. Also, computer models and software help reduce the clinical time and expertise necessary to optimally implement these medical devices. While some UTSCS computer model predictions were already validated in the past, it is still necessary that important output parameters (such as usage range, perception thresholds, and discomfort thresholds) drawn from our modeled triple-lead and AI stimulation studies be tested clinically, allowing for a synergistic analysis of results. Such analyses can be of high relevance, especially when considering the large inter-subject anatomical variability of the spinal cord within human subjects. Also more knowledge of this neuroanatomy and its variability is required. As a subsequent step, close interdisciplinary collaboration is essential in order to direct future research and provide in-depth understanding of the clinical effects of triple-lead longitudinal and transverse tripolar stimulation (using both percutaneous and surgical leads), and AI technique of longitudinal tripoles (LGC and LGC+) on spinal nerve fibers. In the near future, as SCS equipments evolve to incorporate novel features (such as improved lead and IPG designs, improved electrode contact combinations, robust programming capabilities etc.) to allow for better customization of 
the therapy to the patient, they will undoubtedly need advanced, flexible, validated computational models and software to effectively implement the same. Also, due to the fact that no computer model can be perfectly accurate, and since every patient's pain complaint and subsequent response to stimulation is bound to be different; an adaptive approach might be necessary to fine-tune the therapy. Future research should focus on delivering optimized stimulation patterns to the spinal cord using adaptive electrode array designs that can interface computers to damaged nerve fibers.

We believe that the results from our computer modeling study and many more will contribute and supplement to the importance of stimulation optimization techniques in SCS, using both percutaneous and surgical lead approaches, and consequently lead to increased effectiveness of SCS therapy in chronic pain relief. Just as it is important for engineers developing implantable percutaneous and surgical triple-lead electrode arrays in SCS to understand their functions, it is equally important for the clinician using them to realize and interpret how these arrays operate, the trade-offs involved in their design and the capabilities and limitations of the technology. This mutual understanding allows for enhanced electrode design and optimization on the part of the engineer and optimal prescription and programming by the physician. In order to further investigate the stimulation optimization techniques in SCS for chronic pain conditions, improved attention for interaction between the clinicians and scientists is essential. Specific questions generated by the clinician should furnish research problems for the scientist who has the means to test the respective ideas in well-controlled systems. This collaboration can result in further improvements and breakthroughs in SCS technology and therapy. 


\section{References}

[1] North RB, Ewend MG, Lawton MT, Piantadosi S. Spinal cord stimulation of chronic, intractable pain: superiority of 'multi-channel' devices. Pain. 1991;44:119-130.

[2] Smith MC, Deacon P. Topographical anatomy of the posterior columns of the spinal cord in man. The long ascending fibers. Brain. 1984;107:671-698.

[3] Aló KM, Redko V, Charnov J. Four year follow-up of dual electrode spinal cord stimulation for chronic pain. Neuromodulation. 2002;5:79-88.

[4] North RB, Brigham DD, Khalessi A, Calkins SK, Piantadosi S, Campbell DS. Spinal cord stimulator adjustment to maximize implanted battery longevity: a randomized controlled trial using a computerized, patient-interactive programmer. Neuromodulation. 2004;7:13-25.

[5] Caraway D, Miyazawa G, Greenberg J, King G. A midline single cathode offers preferential dorsal column recruitment with spinal cord stimulation. Anesthesiology. 2006;A172.

[6] North RB, Kidd DH, Zahurak M. Spinal cord stimulation for chronic, intractable pain:experience over two decades. Neurosurgery. 1993;32:384-395.

[7] Struijk JJ, Holsheimer J. Transverse tripolar spinal cord stimulation: theoretical performance of a dual channel system. Med Biol Eng Comput. 1996;34:273-279.

[8] Kumar K, Lind G, Winter J. Spinal cord stimulation: placement of surgical leads via laminotomytechniques and benefits. In: Krames ES, Peckham PH, Rezai AR, eds. Neuromodulation, Vol 2. Elsevier, New York: 2009;1005-1012.

[9] Renard VM, North RB. Prevention of percutaneous electrode migration in SCS by a modification of stanfard implantation technique. J Neurosurg Spine. 2006;4:300-303.

[10] Buvanendran A and Lubenow T J. Efficacy of transverse tripolar spinal cord stimulator for the relief of chronic low back pain from failed back surgery Pain Physician. 2008;11:333-338.

[11] Villavicencio A, Leveque J, Rubin L, Bulsara K and Gorecki J. Laminectomy versus percutaneous electrode placement for spinal cord stimulation Neurosurgery. 2000;46:399-406.

[12] North RB, Kidd DH, Olin J, Sieracki JM, Farrokhi F, Petrucci L et al. Spinal cord stimulation for axial low back pain: a prospective, controlled trial comparing dual with single percutaneous electrodes. Spine. 2005; 30:1412-1418.

[13] Richter E, Abramova M, Alo K. Low back paresthesia coverage with lateral programming of fivecolumn paddle leads: technical report. J Neurosurg. 2011;64-68. 
Chapter 6 


\section{Summary}

The primary indication of SCS is chronic pain, in particular neuropathic pain. It is a highly debilitating condition, and in particular, is estimated to affect about one-fifth of the population in Europe (18\% in the Netherlands). SCS, which uses electrical stimulation, is a valuable treatment for chronic intractable neuropathic pain. It aims at improving the quality of life of chronic pain patients, by decreasing the pain intensity and substituting it with a tingling paresthesia sensation. The overall success rate of this treatment modality is about $70 \%$. As a potential improvement, this thesis presents the clinical and technical aspects of stimulation optimization techniques for chronic pain relief in SCS. The optimization techniques are aimed to focus primarily on improving SCS equipment. In particular, the thesis investigates the performance of novel percutaneous and surgical triple-lead configuration designs, with both longitudinal and transverse tripolar contact combinations, in a current-controlled stimulation approach. Effects of percutaneous lead alignment/misalignment, varied transversal lead spacing, preferred choice of leads (surgical/percutaneous), and IPG design are also modelled as ways to potentially improve SCS equipment.

In Chapter 1, SCS is presented as one of the main treatment modalities for chronic pain suppression. Details are given on SCS background and mechanisms of action, its indications, equipment design, procedure and efficacy. Clinical and technical aspects such as choice of current/voltage stimulation, lead number, lead positioning and lead contact combinations are also reviewed with recommendations for further improvements that may enhance the effectiveness of the therapy.

In Chapter 2, triple percutaneous leads programmed to function as longitudinal guarded cathodes are modelled as a potential improvement to dual leads commonly used in clinical practice. The effect of transversal lead separation and anodal current steering mechanisms using a triple lead guarded cathode configuration on the medio-lateral extent of DC coverage is studied. Reducing the transverse lead separation resulted in increasing the depths and widths of the recruited DC area. The triple lead configuration with the least transverse separation had the largest DC recruited area and usage range. Also, the post-operative flexibilities of single, dual and triple lead longitudinal guarded cathode configurations are compared.Triple leads programmed to perform as longitudinal guarded cathodes provide more postoperative flexibility than single and dual leads in covering a larger width of the low-thoracic DCs

In Chapter 3, electrode alignment of transverse tripoles using a percutaneous triple lead approach is modelled. The influence of electrode alignment of the transverse tripoles on the paresthesia coverage of pain area is presented. Aligned and staggered triple leads are modelled and transverse tripolar stimulation 
is performed to investigate the effects of the above configurations on the DC recruited area. The aligned transverse tripoles facilitated deeper penetration into the medial dorsal columns (DCs) and allow selective targeting of either medial or lateral DC fibers,. The staggered transverse tripoles always enabled broad and bilateral DC activation, at the expense of medio-lateral steerability. Steering of anodal currents between the lateral leads of the staggered transverse tripoles cannot target medially-confined populations of DC fibers in the spinal cord. An aligned transverse tripolar configuration is strongly recommended, because of its ability in providing more post-operative flexibility than other configurations.

In Chapter 4, transverse tripolar configurations using quadripolar instead of dual anodes are modelled both using percutaneous and surgical leads. The additional anodal contacts are programmed to understand the stimulation effects on DC fiber selectivity and shielding of DR fibers. The percutaneous transverse tripolar configuration with quadripolar anodes improved the depth of DC penetration and enabled selective recruitment of DCs as compared to the percutaneous staggered configuration with dual anodes. The effect of contact spacing and insulation is determined by comparing the performance of the percutaneous and surgical triple lead transverse tripolar configurations with quadripolar anodes. Our modeling study hypothesizes that, in clinical practice, the surgical configuration with quadripolar anodes can provide an improved performance compared to the percutaneous configuration. Our model also predicts that the same configuration realized on the commercial surgical lead with widely-spaced contacts cannot selectively stimulate DCs essential in treating low-back pain.

Chapter 5 introduces and investigates anode intensification effects on the performance of transverse tripolar and longitudinal tripolar configurations. Anodal currents are increased with respect to the cathode to determine the effects of stimulation on DC recruitment and usage ranges. Transverse tripolar stimulation with anode intensification recruited a smaller depth and width of medial DC recruited area as compared to the situation with no anode intensification. Therefore, anode intensification of transverse tripoles is not advantageous, as the same DC recruitment can be achieved at lower stimulation amplitudes with balanced anodal and cathodal currents. In contrast, anode intensification of longitudinal guarded cathodes resulted in increasing the depths and widths of the recruited DC area respectively. This may increase the likelihood of activating fibers inaccessible with conventional programming.

Also, anode intensification of longitudinal guarded cathodes with wider contact spacing recruited a larger DC area and usage range as compared to that of the configuration without anode intensification.

In Chapter 6 of the thesis, the clinical and technical aspects of stimulation optimization techniques for chronic pain relief in SCS are discussed. The optimization techniques focussed primarily on improving 
SCS equipment-lead design, lead placement and alignment, lead choice and implantable pulse generator design. In addition, validity of the model and future outlook were also discussed. 
Summary and Samenvatting 


\section{Samenvatting}

Chronische pijn en in het bijzonder neuropathische pijn is de primaire indicatie voor ruggenmerg stimulatie (spinal cord stimulation, SCS). Het is een zeer belastende aandoening en treft naar schatting een vijfde deel van de Europese bevolking (18\% in Nederland). Ruggenmergstimulatie, waarbij gebruik wordt gemaakt van elektrische stimulatie, is een waardevolle behandeling voor chronische pijn die op andere wijze onbehandelbaar is. Het is bedoeld om de kwaliteit van leven te verbeteren door de pijn te onderdrukken en in het pijnlijke gebied een tintelende sensatie (paresthesie) op te wekken. De kans op succesvolle behandeling met SCS is ongeveer 70\%. Ter verdere verbetering van SCS behandelingen worden in dit proefschrift klinische en technische aspecten voor verbetering van de stimulatietechniek gepresenteerd. Deze optimalisatietechnieken richten zich primair op verbetering van de apparatuur. Met name de prestaties van nieuwe ontwerpen voor percutane en chirurgische elektrode 'triple lead' configuraties, met zowel longitudinale als transverse tripolaire contact combinaties worden in dit proefschrift onderzocht voor stroomgestuurde stimulatiecondities. Daarnaast worden gemodelleerde effecten van onderlinge (mis)uitlijning bij percutane leads, variërende transversale afstanden tussen leads, keuze voor lead type (percutaan of chirurgisch) en ontwerp van de implanteerbare pulsgenerator geëvalueerd als potentiële verbetering van SCS.

In hoofdstuk 1 wordt SCS gepresenteerd als één van de belangrijkste methoden voor bestrijding van chronische pijn. De achtergronden van SCS worden belicht, zoals werkingsmechanismen, indicaties voor toepassing, ontwerp van apparatuur, procedures en effectiviteit. Daarnaast wordt een overzicht gegeven van klinische en technische aspecten, zoals keuze voor spanning- of stroomgestuurde stimulatie, aantal en positionering van de leads, contact combinaties, en worden aanbevelingen voor verdere verbeteringen belicht die de effectiviteit van de behandeling zouden kunnen verhogen.

In hoofdstuk 2 wordt een modelstudie gepresenteerd waarin de potentiële verbeteringen van drievoudige percutane leads, geprogrammeerd als longitudinale 'guarded cathodes', wordt vergeleken met de klinisch veel toegepaste dubbele leadconfiguratie. De studie richt zich op het effect van falende onderlinge uitlijning van en afstanden tussen transversale leads en anodale stroomsturing bij drievoudige 'guarded cathode' configuraties op de mediolaterale activatie van dorsale kolommen (dorsal colunms, DC). Verkleining van de transversale lead afstand resulteert in een toename van de diepte en mediolaterale reikwijdte van DC activatie. Met de kleinste transversale lead afstand blijkt het grootste gebied op te treden waarbinnen DC zenuwvezels geactiveerd kunnen worden en ontstaat tevens het grootste bruikbare stimulatiebereik (usage range). Daarnaast zijn de post-operatieve flexibiliteit van enkelvoudige, duale en drievoudige longitudinale 'guarded cathodes' vergeleken. Met drievoudige leads bleek de post-operatieve 
flexibiliteit met betrekking tot het bereiken van goede mediolaterale reikwijdte en diepte van DC activatie het grootst.

In hoofdstuk 3 wordt het effect van uitlijning van percutane leads op de prestaties van transversale tripolaire configuraties gemodelleerd. Belangrijkste uitkomstmaat hierbij is de bedekking van het pijnlijke gebied door de opgewekte paresthesie die bepaald wordt door de activatie van zenuwvezels in de dorsale colommen. Simulaties betreffen zowel volledig uitgelijnde als verschoven anodale contacten. Transversale tripolen met uitgelijnde contacten blijken dieper gelegen zenuwvezels in de mediale dorsale colommen te activeren. Daarbij maakt het sturen van de anodale stroomverdeling over de laterale contacten selectieve activatie van mediale of laterale zenuwvezels mogelijk. Verschoven anodes leiden tot verbreding van het geactiveerde DC gebied, ten koste van mediolaterale selectiviteit: een uitsluitend mediaal activatiegebied is niet langer mogelijk. Uitlijning van transverse tripolaire configuraties verdient aanbeveling omdat het leidt tot meer post-operatieve flexibiliteit.

In hoofdstuk 4 worden transverse tripolaire configuraties met quadripolaire in plaats van duale anodes gemodelleerd, zowel voor percutane als voor chirgurgische leads. Twee additionele anodale contacten zijn toegevoegd om hun effect op enerzijds de selectieve activatie van DC zenuwvezels en anderzijds op de anodale afscherming (shielding) van dorsale wortel (Dorsal Root, DR) vezels beter te begrijpen. De percutane transversale tripolaire configuratie met quadripolaire anodes verbeterde de selectiviteit en diepte van DC activatie in vergelijking tot de percutane transversale tripool met duale verschoven anodes. Het effect van contact afstand en elektrisch isolerend materiaal op transversale tripolen met quadripolaire anodes is onderzocht door bij deze configuratie de prestaties van percutane leads te vergelijken met chirugische leads. De simulaties suggereren dat in de klinische praktijk quadripolaire anodes bij chirurgische leads tot betere prestaties zullen leiden dan bij percutane leads. De simulaties suggereren ook dat wanneer deze configuratie gerealiseerd wordt middels commercieel verkrijgbare chirurgische leads met grotere contact afstanden, de DC selectiviteit benodigd voor behandeling van lage rugpijn niet gehaald kan worden.

In hoofdstuk 5 wordt het concept 'anode intensificatie' geïntroduceerd en het effect hiervan op de prestaties van transversale tripolaire en longitudinale 'guarded cathode' configuraties onderzocht. In het SCS model worden hiertoe de anodale stromen vergroot in vergelijking tot de cathodale stroom en wordt het resulterende DC activatie gebied en het bruikbare stimulatiebereik gesimuleerd. Transverse tripolaire stimulatie met anode intensificatie leidt tot een ondiepere activatie van DC vezels in vergelijking tot stimulatie met gebalanceerde anodale stromen. In deze situatie levert anode intensificatie geen voordeel omdat eenzelfde DC activatie bereikt kan worden met gebalanceerde anodale stromen bij een lagere 
intensiteit. Bij longitudinale 'guarded cathode'configuraties leidt anode intensificatie tot een grotere diepte en mediolaterale breedte van het gebied waarin DC vezels geactiveerd kunnen worden. Dit vergroot de mogelijkheden tot het activeren van DC vezels die middels conventionele (gebalanceerde) stimulatie niet geactiveerd kunnen worden. Daarbij leidt anode intensificatie ook bij een grotere contact afstand tot een groter DC activatie gebied en bruikbaar stimulatiebereik.

In hoofdstuk 6 worden de klinische en technische aspecten van de stimulatie optimalisatie technieken voor bestrijding van chronische pijn middels SCS bediscussiëerd. De optimalisaties waren vooral gericht op verbetering van type, ontwerp, plaatsing en uitlijning van de leads en het ontwerp van de implanteerbare pulsgenerator. Daarbij worden ook de validiteit van het SCS model en toekomstig onderzoek besproken. 
Summary and Samenvatting

122 


\section{Curriculum Vitae}

Vishwanath was born on the $1^{\text {st }}$ of July 1981 in Chennai, India.

Having completed High school as a Science student he joined the Faculty of Mechanical engineering at the University of Madras (now Chennai). Soon after receiving his Bachelor degree in Mechanical Engineering from the same University, he flew to Aachen, Germany in 2003 to pursue his Master studies. He graduated as a Master of Science in Biomedical Engineering in 2006 after defending his thesis titled 'Characterization of neural network response to electrical stimulation on microelectrode arrays for the development of a biosensor'. Between 2006 and 2007, he worked as a research assistant in the Neurology department at the Uniklinikum, Dusseldorf.

From June 2007, he started working as a PhD at Biomedical Signals and Systems (BSS) group of the University of Twente, Netherlands. The work presented here is an outcome of his four years of research. 


\section{List of publications}

Sankarasubramanian V, Buitenweg JR, Holsheimer J, Veltink P. Electrode alignment of transverse tripoles using a percutaneous triple-lead approach in spinal cord stimulation. J. Neural Eng. 2011. doi:10.1088/1741-2560/8/1/016010.

Sankarasubramanian V, Buitenweg JR, Holsheimer J, Veltink P. Triple leads programmed to function as longitudinal guarded cathodes in spinal cord stimulation: a modeling study. Neuromodulation. 2011;14:401-411.

Sankarasubramanian V, Buitenweg JR, Holsheimer J, Veltink P. Staggered transverse tripoles with quadripolar lateral anodes using percutaneous and surgical leads in SCS (Accepted in Neurosurgery). 2012. doi: 10.1227.

\section{Manuscript submitted for publication:}

Sankarasubramanian V, Buitenweg JR, Holsheimer J, Veltink P. Performance of transverse tripoles vs longitudinal tripoles with anode intensification: computational modelling study.

\section{Abstracts, posters and oral presentations at meetings and international conferences:}

Sankarasubramanian V, Buitenweg JR. Triple percutaneous leads with aligned and staggered transverse tripoles in spinal cord stimulation. $1^{\text {st }}$ Joint Neuromodulation Meeting Benelux, Groningen, The Netherlands, January 2011 (abstract and poster).

Sankarasubramanian V, Buitenweg JR. Triple percutaneous leads with transverse tripoles: a modeling study. $3^{\text {rd }}$ Dutch on Biomedical Engineering, Egmond aan Zee, The Netherlands, January 2011 (abstract and oral presentation).

Sankarasubramanian V, Buitenweg JR. Triple percutaneous leads with aligned and staggered transverse tripoles in spinal cord stimulation. Joint Congress of the French, German, Italian, Southeastern Europe and Spanish Neuromodulation, Madrid, Spain, June 2012 (abstract and poster).

Sankarasubramanian V, Buitenweg JR, Holsheimer J. Mediolateral field steering in spinal cord stimulation using triple leads with longitudinal guarded cathodes. INS-9 ${ }^{\text {th }}$ World Congress, Seoul, Korea, September 2009 (abstract and oral presentation). 
List of publications 